PREPARED For SUbMission to JHEP

\title{
Holographic RG Flows from Quasi-Topological Gravity
}

\author{
G.M. Sotkov ${ }^{a}$ and U. Camara $\mathbf{d S}^{a}$ \\ ${ }^{a}$ Universidade Federal do Espítiro Santo, \\ Espírito Santo, Vitória, Brazil \\ E-mail: gsotkov@gmail.com, ulyssescamara@gmail.com
}

\begin{abstract}
We investigate the holographic Renormalization Group (RG) flows and the critical phenomena that take place in the QFT's dual to the d-dimensional cubic QuasiTopological Gravity coupled to scalar matter. The knowledge of the corresponding flat Domain Walls (DWs) solutions, allows us to derive the explicit form of the QFT's beta -functions, as well as of the trace anomalies $a(l)$ and $c(l)$, in terms of the matter superpotential. As a consequence we are able to determine the complete set of $C F T$ data characterizing the universality classes of the UV and IR critical points and to follow the particular RG evolution of this data. We further analyse the dependence of the critical properties of such dual QFT's on the values of the Lovelock couplings and on the shape of the superpotential. For odd values of $d$, the explicit form of the " $a$ and $c$-central charges" as functions of the running coupling constant, enable us to establish the conditions under which the $a / c$-Theorems for their decreasing are valid. The restrictions imposed on the massless Holographic RG flows by the requirements of the positivity of the energy fluxes are derived. The particular case of quartic Higgs-like superpotential is studied in detail. It provides an example of unitary dual QFT's having few $c \neq a$ critical points representing second or infinite order phase transitions. Depending on the range of the values of the coupling constant they exhibit massive and massless phases, described by a chain of distinct DWs solutions sharing common boundaries.
\end{abstract}




\section{Contents}

1 Introduction 1

2 CFT-data from QT Gravity DWs 4

2.1 Superpotential and Lovelock's vacua 5

$\begin{array}{lll}2.2 & \text { Renormalization Group and CFT-data } & 7\end{array}$

$\begin{array}{ll}2.3 \text { Weyl anomalies and central functions } & 11\end{array}$

3 Holographic $a / c$-Theorems $\quad 16$

$\begin{array}{ll}3.1 \text { On the content of } a / c \text { - Theorems } & 16\end{array}$

$\begin{array}{ll}3.2 & \text { On the methods of proof } \\ 3.3 & 18\end{array}$

$3.3 d=5$ Proof: Standard $a / c$-Theorem 20

$\begin{array}{lll}3.3 .1 & \mu_{+} \text {model } & 20\end{array}$

3.3.2 $\mu_{-}$model 23

3.3.3 Topological versus $a$-scales $\quad 24$

3.4 Comments on $d=5 c$-Modified and $a$-Weaken Theorems 25

4 Gauss-Bonnet $a / c$-Theorems $\quad 26$

5 Holographic $d \geq 7 a / c$ - Theorems $\quad 28$

$5.1 \mu_{ \pm}$models Standard Theorem 28

$\begin{array}{ll}5.2 & \text { Maximal scale and Modified } c \text {-Theorem } \\ \end{array}$

$6 \quad$ Energy Fluxes Positivity and $a / c$-Theorems 31

6.1 More on $C F T_{d-1}$ data: positive energy fluxes conditions 31

6.2 Holographic GB $t_{2}$-Theorem 33

6.3 New minimal scales in $d=5$ QT Gravity $a / c$-Theorems 35

7 Holographic RG Flows and Phase Transitions $\quad 41$

7.1 On the Off-Critical Holography Dictionary 41

7.2 Quartic Higgs-like Superpotential 44

7.3 Phase Transitions in QFT's duals to GB Gravity 45

7.3.1 Negative $\lambda$ models: massless-to-massive phase transition 46

$\begin{array}{ll}\text { 7.3.2 Positive } \lambda \text { models: massless RG flows } & 49\end{array}$

7.4 RG Flows from Quasi-Topological Gravity DW's $\quad 51$

$\begin{array}{lll}\text { 7.4.1 Minimal scale phase structures } & 53\end{array}$

$\begin{array}{ll}\text { 7.4.2 } & \text { Maximal scale massless phase } \\ \end{array}$

$\begin{array}{lll}8 & \text { Conclusions } & 57\end{array}$ 
A The proof of $d \geq 7 a / c$ - Theorems

A.1 $\mu_{+}$model 63

$\begin{array}{lll}\text { A.2 } \mu_{-} \text {model } & 65\end{array}$

$\begin{array}{ll}\text { B GB domain walls for negative } \lambda & 66\end{array}$

\section{Introduction}

According to the gauge/gravity correspondence rules, the holographic description of certain strongly coupled $Q F T_{d-1}$ at infinite volume and zero temperature is based on the particular flat Domain Walls (DW's) solutions of their dual d-dimensional Gravity models [1-4]. The problem addressed in the present paper concerns the systematic application of the Gauss-Bonnet (GB) and cubic Quasi-Topological Gravity (QTG) DW's [5, 6] to the investigation of the Renormalization Group properties and of the phase structure of their $Q F T_{d-1}$ 's dual. It represents an off-critical extension of the recent holographic studies [7-11] of a family of $a \neq c C F T_{d-1}$ 's with non-vanishing energy flux parameter $t_{4}$. Special attention is devoted to the unitarity and causality of the massless RG flows described by these DW's solutions. The explicit proof of the corresponding holographic " $a$ and $c$ - Theorems" $[3,12,13]$, together with the implementation of the energy fluxes positivity requirements [14-16] on the UV- and IR-CFT's data, allows us to select the physically consistent "chains" of DW's and to establish a set of conditions on the parameters of the matter superpotential $W(\sigma)$. The aim of the present research is to provide more arguments confirming the conjecture that by holographic calculations based on $d=5$ GB and cubic QT Gravity models $[17,18]$ one can reproduce the strong coupling limits of certain unitary (non-supersymmetric) $Q F T_{4}$ 's, whose massive and massless phases exhibit "phenomenologically reasonable" off-critical features.

Domain walls. The flat static domain walls of $A d S_{d}$-type represent a special class of $S O(d-2,1) \rtimes T_{d-1}$ symmetric solutions $[5,6,20]$ of $d$-dimensional Einstein (super)gravity and its "higher derivatives" generalizations [17-19] of negative cosmological constant $\Lambda<0$ coupled to an appropriate scalar matter of potential $V(\sigma)$. They are usually defined by the following ansatz:

$$
\begin{aligned}
d s_{d}^{2} & =g_{\mu \nu}\left(x_{\rho}\right) d x^{\mu} d x^{\nu}=d y^{2}+e^{2 A(y)} \eta_{i j} d x^{i} d x^{j}, & \mu, \nu=0,1,2 \ldots, d-1 \\
\eta_{i j}=(-,+, \ldots,+), & i, j=0,1 \ldots, d-2, & \sigma\left(x_{i}, y\right)=\sigma(y)
\end{aligned}
$$

together with the standard DW's boundary conditions (b.c.):

$$
e^{A}(y \rightarrow \pm \infty) \approx e^{\frac{y}{L_{ \pm}}}, \quad \sigma( \pm \infty)=\sigma_{ \pm}^{*}, \quad V^{\prime}\left(\sigma_{ \pm}^{*}\right)=0 .
$$

Such DW's describe asymptotically $A d S_{d}$ space-times $^{1}$, whose main feature is that the matter energy is concentrated around certain $(d-2)$-dimensional subspaces dividing the

\footnotetext{
${ }^{1}$ denoted as $(a) A d S_{d}$ in what follows
} 
(a) $A d S_{d}$ space-time in two parts of different $A d S_{d}$ vacua $[5,20,21]$ related to the extrema of the matter potential.

Off-critical Holography. The increasing interest in the construction of exact GB and QTG DW's solutions is mainly motivated by their role in the description of the holographic Renormalization Group flows [3, 9, 22] between specific conformal field theories $C F T_{d-1}^{U V / I R}$ dual to two consecutive $A d S_{d}$-vacua of different small cosmological constants $\Lambda_{U V / I R}=-(d-1)(d-2) / 2 L_{U V / I R}^{2}$. The off-critical holography thus offers a nonperturbative information about certain non-conformal (sypersymmetric) $Q F T_{d-1}$ 's of few "critical UV/IR-points", that are conjectured to be dual to the considered GB and cubic Quasi-Topological Gravity-matter models [17, 18]. It is convenient to realize them as appropriate $C F T_{d-1}^{U V}$ 's perturbed by certain relevant operators $\Phi_{\sigma}\left(x^{i}\right)$, which break the conformal $S O(d-1,2)$ symmetry to its Poincare subgroup $S O(d-2,1) \rtimes T_{d-1}[2,3]$. The actions of such QFT's can be written in the form of perturbed $C F T$ 's [12, 33]:

$$
S_{Q F T_{d-1}}(\sigma)=S_{C F T_{d-1}}^{U V}+\left(\sigma\left(L_{r g}\right)-\sigma_{U V}\right) \int d^{d-1} x \Phi_{\sigma}\left(x^{i}\right)
$$

Then the "running" coupling constant $\sigma\left(L_{r g}\right)$ of the dual $Q F T_{d-1}$ is identified with the scalar field $\sigma(y)$ and the RG "energy scale" $L_{r g}^{2}$ is simply related to the scale factor $e^{2 A(\sigma)}$ of certain d-dimensional extended EH gravity DW's: $A(\sigma)=-l=\ln \left(l_{p l} / L_{r g}(\sigma)\right)[2,3,23]$.

Holographic $a \neq c$ Theorems. Given a pair of dual $(a) A d S_{d} / Q F T_{d-1}$ theories, by applying the well known "holographic rules" [1-3], one can reproduce important strong coupling (i.e. small $\Lambda^{e f f}$ ) features of the quantum $Q F T_{d-1}$ as for example the RG evolution of all the UV conformal data - the $a(l)$ and $c_{n}(l)$ - central functions, the anomalous dimensions $\Delta(\sigma)=d-1-s(\sigma)$ etc. - from the corresponding classical DW's solutions. For odd values of $d$, the knowledge of the explicit form of the " $a$ and $c$ - central charges" as functions of the RG scale $l$, allows to further investigate the conditions under which the Zamolodchikov-Cardy's $a / c$-Theorems $[12,13]$ for their decreasing are valid ${ }^{2}$. It is worthwhile to mention that the DW's of the Einstein (super)gravity of $\Lambda<0$ coupled to matter turns out to lead to very restricted $c=a$ class of $\mathcal{N}=4$ suppersymmetric $C F T$ 's and QFT's, while the CFT's dual to the extended GB and Quasi-Topological gravity-matter models are known to describe a family of (non-)sypersymmetric field theories, having (at least) two distinct central charges $a \neq c$.

One of the important results established in the present paper is the following holographic $a \neq c$ Theorem: in all the $Q F T_{4}$ 's duals to the $d=5 G B$ and cubic QT Gravity of negative matter superpotential $W(\sigma)<0$, having at least two extrema, the $a(l)$ and $c(l)$ -central functions are both positive and decreasing during the massless $R G$ flow. The conditions of its validity turns out to impose a specific lower bound $L_{\min }$ on the values of the UV and IR scales ${ }^{3} L_{U V / I R}$ of the $C F T_{4}^{U V / I R}: L_{U V}>L_{I R}>L_{m i n}$. The exact values of these minimal scales are obtained from the requirements of positivity of the energy fluxes $[7,9,14]$ in the corresponding dual $C F T_{4}^{U V / I R}$, s.

\footnotetext{
${ }^{2}$ see for example ref. [9] for recent discussion.

${ }^{3}$ determined by the central charges $a_{U V / I R}$ and $c_{U V / I R}$, or asymptotically by the number of colors $\left(N_{c}^{2}\right)_{U V} \approx\left(L_{U V} / l_{p l}\right)^{3}$ of the corresponding $S U\left(N_{c}\right) C F T_{4}^{U V / I R}$.
} 
$D W$ 's chains and QFT's phase transitions. The concept and the explicit constructions of chains of standard and singular (involving naked singularities) DW's of common boundaries $[5,6,34]$ are our basic tools in the analysis of the critical phenomena that occur in the considered dual QFT's. The close relationship between the QFT's phase transitions and the existence of chains of distinct gravitational DW's, suggests an interpretation of such couples of DW's as representing geometric phase transitions in the space of all the DW's solutions of the GB and cubic QTG models. We further investigate the dependence of the critical properties of these dual QFT's on the values of the Lovelock couplings, on the shape of the superpotential and on the scalar field $\sigma$ boundary conditions compatible with the unitarity and causality requirements.

$Q C D_{4}$ and $s Q G P$-hydrodynamics applications? The relevance of certain $a \neq c$ fourdimensional CFT models dual to $d=5$ extended EH gravity, in the description of the hydrodynamics of strong coupled quark-gluon plasma (sQGP) $[24-26]^{4}$, motivates the recent intensive investigations of the effects of "higher curvature" gravitational interactions on the intrinsic unitarity and causality properties of their dual $C F T_{4}$ 's $[8,10,11,14]$. At the energy scales of experimental interest, the realistic sQGO models, based on the (supersymmetric) $Q C D_{4}$ are however known to be non-conformal $Q F T$ 's. Therefore in this holographic (a)AdS $S_{5} / Q F T_{4}$ context, the problem of construction of appropriate classical solutions of the extended $d=5 \mathrm{~GB}$ and Quasi-Topological gravity-matter models as for example DW's and of their finite temperature analogues called thermal gas solutions [71], becomes of real practical importance [63].

One relevant result concerning the RG behaviour of the ratio $\eta / s$ is provided by the $\frac{a}{c}$-Theorem, established in Sect.6 in the case of (zero temperature) massless RG flows in the $Q_{F T}$ 's dual to $d=5 \mathrm{~GB}$ gravity-matter model. It suggests that for positive GB couplings $\lambda$, the corresponding UV- and IR- values of $\eta / s$ satisfy the following inequality: $(\eta / s)_{U V} \geq(\eta / s)_{I R}$, while for negative $\lambda$ we find an indication that $\eta / s$ should be increasing. The complete description of the off-critical behaviour of $\eta / s$ as a function of the RG scale (or of the coupling constant $\sigma$ ) in the massive and massless phases of the dual $Q_{F T}$, requires further investigations.

The layout of paper. In Sect.2.1. we review some basic concepts and results concerning the cubic QT Gravity vacua structure, its DW's solutions and the superpotential method. Sects.2.2. and 2.3. contain a brief summary of few important $C F T_{d-1}$ 's and renormalization group topics, involving the definitions of the scaling dimensions, central charges, OPE's and their relations to the holographic $\beta(\sigma)$-function. Our proofs of the holographic " $a$ and $c$ - Theorems" for the $Q F T_{d-1}$ dual to GB and cubic QT Gravity coupled to scalar matter, together with the specific restrictions on the values of the UV and IR scales $L_{U V / I R}$ and on the Lovelock's gravitational couplings $\lambda$ and $\mu$ necessary for their validity are presented in Sects.3,4,5 and App. A. We derive in Sect.6 the additional "stronger" requirements imposed on the physical scales $L_{U V / I R}$ by the positivity of the energy fluxes at the $C F T_{d-1}$ 's, representing the UV and IR critical points. Sect.7 is devoted to the detailed study of the

\footnotetext{
${ }^{4}$ in particular the evidences for possible violations of the "universal lower bound" $\eta / s=1 / 4 \pi$ of the ratio of shear viscosity to entropy density for such "conformal fluids".
} 
critical phenomena in a specific family of $Q F T$ 's, corresponding to a particular choice of quartic Higgs-like matter superpotential. They provide examples of dual $Q F T_{d-1}$ 's having few $c \neq a$ critical points of second and infinite order phase transitions and specific massive and massless phases. Few important results concerning the explicit form and the properties of the GB domain walls for negative values of $\lambda$, that are important in the description of the massless-to-massive phase transitions in the dual QFT's (see Sect.7.3.), are derived in our App.B.

\section{CFT-data from QT Gravity DWs}

We are interested in the off-critical properties of the $Q F T_{d-1}$ dual to the cubic QT Gravity $[17,18]$ coupled to scalar matter:

$$
\begin{aligned}
S_{G B L}^{\mathrm{eff}} & =\frac{1}{\kappa^{2}} \int d^{d} x \sqrt{-g}\left[R+\frac{\lambda L^{2}}{(d-3)(d-4)}\left(R_{a b c e} R^{a b c e}-4 R_{a b} R^{a b}+R^{2}\right)\right. \\
& \left.-\frac{8 \mu(2 d-3) L^{4}}{(d-6)(d-3)\left(3 d^{2}-15 d+16\right)} \mathcal{Z}_{d}-\kappa^{2}\left(\frac{1}{2} g^{a b} \partial_{a} \sigma \partial_{b} \sigma+V(\sigma)\right)\right],
\end{aligned}
$$

where $\kappa^{2}=\frac{8 \pi^{(d-1) / 2}}{\Gamma((d-1) / 2)} G_{d}$ is defining the d-dimensional Plank scale, the $L^{2}$ is representing the new scale, related to the bare cosmological constant ${ }^{5}$, specific for the "higher order" gravitational models [27], while $\lambda$ and $\mu$ denote the appropriately normalized dimensionless Gauss-Bonnet and Lovelock "gravitational" couplings. The cubic $\mathcal{Z}_{d}$-invariant [17] has the specific form:

$$
\begin{aligned}
& \mathcal{Z}_{d}=R_{a b^{c}{ }^{d}} R_{c}{ }^{e}{ }^{f}{ }^{f} R_{e}{ }^{a} f^{b}+\frac{1}{(2 d-3)(d-4)}\left[\frac{3(3 d-8)}{8} R_{a b c d} R^{a b c d} R-3(d-2) R_{a b c d} R^{a b c}{ }_{e} R^{d e}+\right. \\
& \left.+3 d R_{a b c d} R^{a c} R^{b d}+6(d-2) R_{a}{ }^{b} R_{b}{ }^{c} R_{c}{ }^{a}-\frac{3(3 d-4)}{2} R_{a}{ }^{b} R_{b}{ }^{a} R+\frac{3 d}{8} R^{3}\right],
\end{aligned}
$$

ensuring that the equations of motion for the black hole solutions are of second order. In general this action leads to fourth order equations [17, 18]. For conformally flat metrics it reduces to the action of d-dimensional extension of the cubic NMG model [36], derived in ref. [5] by requiring that the corresponding DW's (1.1) obey second order equations as well.

In order to make self-consistent our investigation of the Holographic RG flows derived from certain QT Gravity domain walls, we review in this section the basic concepts concerning the QTG vacua and DW's properties and the most important ingredients in the description of the dual $C F T_{d-1}$ 's - as scaling dimensions, central charges and OPE's, together with their relations to the corresponding quantities in the Wilson and holographic RG approach to the critical phenomena. Subsection 2.1. contains a short summary of the recent results on the GB and QT Gravities DW's [5], while in subsections 2.2. and 2.3

\footnotetext{
${ }^{5}$ The negative bare cosmological constant $\Lambda$ is implicitly defined by the vacuum value of the matter potential $\kappa^{2} V\left(\sigma^{*}\right)=2 \Lambda=-(d-1)(d-2) / L^{2}$ and should be distinguished from the effective cosmological constant $\Lambda^{\mathrm{eff}}$.
} 
we give a brief introduction to some selected topics in CFT's, that are essential in the derivation of the holographic " $a \neq c$ - Theorems" and of the corresponding positive energy fluxes requirements.

\subsection{Superpotential and Lovelock's vacua}

The family of stable flat DWs (1.1) solutions of the cubic Quasi-Topological Gravity (2.1) can be obtained by solving the following $I^{\text {st }}$ order system of equations ${ }^{6}$ :

$$
\dot{\sigma}=\frac{2}{\kappa} W^{\prime} C_{0}(W), \quad \dot{A}=-\frac{\kappa}{d-2} W(\sigma), \quad C_{0}(W)=1-2 \lambda \frac{\kappa^{2} L^{2} W^{2}}{(d-2)^{2}}-3 \mu \frac{\kappa^{4} L^{4} W^{4}}{(d-2)^{4}},
$$

derived by an appropriate extension $[5,6]$ of the superpotential method [20]. The relation between the matter potential $V(\sigma)$ and the superpotential $W(\sigma)$ is given by:

$$
\kappa^{2} V(\sigma)=2\left(W^{\prime}\right)^{2} C_{0}^{2}(W)-\left(\frac{d-1}{d-2}\right) \kappa^{2} W^{2}\left(1-\lambda \frac{\kappa^{2} L^{2} W^{2}}{(d-2)^{2}}-\mu \frac{\kappa^{4} L^{4} W^{4}}{(d-2)^{4}}\right)
$$

Let us remind the common property of all the "higher curvature" generalizations of EH gravity, namely that the effective cosmological constants related to the vacuum values $\sigma_{k}^{*}$ $\left(V^{\prime}\left(\sigma_{k}^{*}\right)=0\right)$ of the superpotential:

$$
f_{k} \equiv L^{2} / L_{k}^{2}=L^{2} \dot{A}^{2}\left(\sigma_{k}^{*}\right)=\kappa^{2} L^{2} W^{2}\left(\sigma_{k}^{*}\right) /(d-2)^{2}
$$

are different from the corresponding "bare" ones

$$
h_{k} \equiv L^{2} / L_{0 k}^{2}=-L^{2} V\left(\sigma_{k}^{*}\right) /(d-1)(d-2) .
$$

As a consequence of eq. (2.3), they satisfy the following cubic "vacua" equation:

$$
h_{k}=f_{k}\left(1-\lambda f_{k}-\mu f_{k}^{2}\right) .
$$

An important feature of the $A d S_{d}$ type of vacua $\left(h_{k}>0\right.$, or $\left.V\left(\sigma_{k}^{*}\right)<0\right)$ of the considered model is the existence of very special kind of topological vacua ${ }^{7}$ defined by the real solutions of the equation $C_{0}\left(f_{\text {top }}\right)=0$. They represent few extrema of the matter potential $V(\sigma)$ that however are not extrema of the superpotential.

The second kind of Lovelock's vacua $\left(\sigma_{k}^{*}, f_{k}\right)$ are determined by all the extrema of the superpotential $W^{\prime}\left(\sigma_{k}^{*}\right)=0$ with $W\left(\sigma_{k}^{*}\right) \neq 0$. According to ref. [17], the stable physical vacua are selected by the "causality" requirement $C_{0}\left(f_{k}\right)=1-2 \lambda f-3 \mu f^{2}>0$. It excludes all the $f_{k}$ 's with $C_{0}\left(f_{k}\right)<0$ since they lead to the wrong "ghosts-like" sign of the graviton's kinetic terms in the "linearized" (Gaussian) form of the Quasi-topological Gravity action (2.1).

\footnotetext{
${ }^{6}$ we have denoted $W^{\prime}(\sigma)=\frac{d W}{d \sigma}$ and $\dot{\sigma}=\frac{d \sigma}{d y}$

7 one can always find an appropriated range of values of the gravitational couplings $\lambda$ and $\mu$ (not both negative), such that at least one of the vacua of the Lovelock gravity-matter model (2.1) to be of topological nature, as it is demonstrated in Sect.3 of ref. [5].
} 
We next consider the case when $\lambda$ and $\mu$ are not both negative and thus we can have at least one topological vacuum, given by the solutions

$$
f_{\text {top }}^{ \pm}=-\frac{1}{3 \mu}\left(\lambda \mp \sqrt{\lambda^{2}+3 \mu}\right)
$$

of the "topological" vacua equation $C_{0}\left(f_{\text {top }}^{ \pm}\right)=0$. They determine the following particular values of the topological "bare" cosmological constants (and of the related $A d S_{d}$ scales $\left.L_{o, t o p}^{ \pm}\right)$

$$
h_{ \pm}=h\left(f_{ \pm}\right)=\frac{1}{27 \mu^{2}}\left[-\lambda\left(2 \lambda^{2}+9 \mu\right) \pm 2\left(\lambda^{2}+3 \mu\right)^{3 / 2}\right],
$$

It turns out that $h_{ \pm}$represent the extremal values of the $h(f): f_{+}$is a local maximum, while $f_{-}$is a local minimum. By construction the $h_{\text {top }}^{ \pm}$are related to the values of the potential $V\left(\sigma_{\text {top }}^{ \pm}\right)$at two of its extrema and therefore they make a part of the boundary data for the considered gravity-matter model (2.1). This suggests that for each fixed value of $0<\lambda<1 / 3 h_{\text {top }}^{+}$we can consider eq. (2.4) as a quadratic equation for $\mu$, providing two different real ${ }^{8}$ values $\mu_{ \pm}(\lambda)$. Notice that for all the values of $\lambda$ we have $h_{-}\left(\mu_{-}\right)>0$ and $h_{+}\left(\mu_{ \pm}\right)>0$, which allows us to fix the fundamental scale as $L^{2}=L_{0+}^{2}\left(\mu_{+}\right)=L_{0-}^{2}\left(\mu_{-}\right)$(see eq. (2.1)), by normalizing the "bare" topological vacuum : $h_{+}\left(\mu_{+}, \lambda\right)=h_{-}\left(\mu_{-}, \lambda\right)=1$. Our choice consists in taking the smallest topological scale of the $\mu_{+}$model, and the largest one for the $\mu_{-}$model. Then the two distinct gravitational models $\left(\lambda, \mu_{ \pm}\right)$of equal fundamental scale, are defined by the following particular form of the Lovelock coupling $\mu$ as a function of $\lambda$ :

$$
\mu_{ \pm}(\lambda)=\frac{1}{27}\left(2-9 \lambda \pm 2(1-3 \lambda)^{3 / 2}\right) .
$$

According to the analysis of ref. [5], the restrictions on the values of $f_{k}$ (and $h_{k}$ ) which lead to stable physical vacua in the different domains of admissible values of $\lambda$ and $\mu$ can be summarized as follows:

(a) The $\mu_{+}$model: Taking into account its main characteristics given by the extrema of the curve $h\left(\lambda, \mu_{+} ; f\right) \equiv h(f)$ :

$$
\begin{aligned}
& h_{+}=h\left(f_{+}\right)=1, \quad h_{-}=h\left(f_{-}\right)=\frac{4-45 \lambda+108 \lambda^{2}-4(1-3 \lambda)^{3 / 2}}{27 \lambda(1-4 \lambda)^{2}}, \\
& f_{+}=\frac{1}{\lambda}(1-\sqrt{1-3 \lambda}), \quad f_{-}=\frac{1}{3 \lambda(1-4 \lambda)}(1-6 \lambda-\sqrt{1-3 \lambda}) .
\end{aligned}
$$

it is easy to verify that:

(a1) for $\lambda<1 / 4$, the minimum $f_{-}<0$ is negative and therefore the physical conditions $h, C_{0}>0$ require that $0<f_{\text {phys }}<f_{+}$;

(a2) in the interval $1 / 4<\lambda<8 / 27$ both $f_{ \pm}>0$ are positive, but now the $h_{-}$is negative and thus violating ${ }^{9}$ the $A d S_{d}$ stability condition: $h>0$. Therefore the $C_{0}(f)>0$ and $h>0$ requirements are satisfied again in the "smallest scale" physical region: $0<f_{\text {phys }}<f_{+}$;

\footnotetext{
${ }^{8}$ remember that $h_{+}>h_{-}$and therefore $\lambda<1 / 3 h_{+}<1 / 3 h_{-}$assures the reality of $\mu_{ \pm}$below.

${ }^{9}$ Nevertheless one can have stable physical vacua (of largest scale) when

$$
f_{\text {phys }}>f_{0}=-\lambda / 2 \mu_{+}+\sqrt{\left(\lambda^{2}+4 \mu_{+}\right) / 4 \mu_{+}^{2}},
$$

where we have denoted by $f_{0}$ the greatest root of $h(f)=0$ equation.
} 
(a3) for $8 / 27<\lambda<1 / 3$ both the $f_{ \pm}$as well as both $h_{ \pm}$are positive. This case permits two qualitatively different physical regions: one is the usual Gauss-Bonnet-like region given by $0<f_{\text {phys }}<f_{+}$, while the second one, $f_{-}<f_{\text {phys }}$, is of a new type with no upper limit on the values of the effective cosmological constants. The topological vacuum $f_{-}$introduces a maximal scale $L_{-}^{2} \sim f_{-}^{-1}$, restricting the possible values of the physical vacua scales from above.

(b) The $\mu_{-}$model: The corresponding definitions of the physical regions are now given by the extrema of another curve $h\left(\lambda, \mu_{-} ; f\right) \equiv \tilde{h}(f)$ :

$$
\begin{aligned}
& \tilde{h}_{+}=\tilde{h}\left(\tilde{f}_{+}\right)=\frac{4-45 \lambda+108 \lambda^{2}+4(1-3 \lambda)^{3 / 2}}{27 \lambda(1-4 \lambda)^{2}}, \quad \tilde{h}_{-}=\tilde{h}\left(\tilde{f}_{-}\right)=1, \\
& \tilde{f}_{+}=\frac{1-6 \lambda+\sqrt{1-3 \lambda}}{3 \lambda(1-4 \lambda)}, \quad \tilde{f}_{-}=\frac{1+\sqrt{1-3 \lambda}}{\lambda},
\end{aligned}
$$

It has the important property that $\tilde{h}_{+}>0$ for all $\lambda>0$. The physical region of minimal scale for this models is then defined by $0<f_{\text {phys }}<\tilde{f}_{+}$, while the region $\tilde{f}_{-}<f_{\text {phys }}$ represents the vacua of maximal scales $L_{-}^{2}\left(\mu_{-}\right)>L_{\text {phys }}^{2}\left(\mu_{-}\right)$.

The above $A d S_{d}$ type of physical vacua $\left(\sigma_{k}, L_{k}\right)$ provide a set of all the admissible b.c.'s for $\sigma(y)$ and $e^{2 A(y)}$ at $y \rightarrow \pm \infty$ for the corresponding stable QT-Gravity DW's solutions. Their explicit form is given by the solutions of the $I^{\text {st }}$ order eqs. (2.2) that relate two neighbouring vacua (see sect.4 of ref. [5], where the explicit form of such DWs was found).

\subsection{Renormalization Group and CFT-data}

2.2.1. RG-data: $\beta$-function. Given the form of the superpotential $W(\sigma)$ and related to it $I^{s t}$ order system (2.2), the scale-radial duality determines the $\beta$-function of the conjectured dual $Q F T_{d-1}[2-4]$ in terms of the superpotential:

$$
\frac{d \sigma}{d l}=-\beta(\sigma)=\frac{2(d-2)}{\kappa^{2}} \frac{W^{\prime}(\sigma)}{W(\sigma)} C_{0}(W), \quad l=-A(\sigma)=\ln \left(l_{p l} / L_{r g}(\sigma)\right)
$$

The constant solutions $\sigma_{k}^{*}$ of the above RG equation (2.8) are defined by the zeros of the $\beta$-function and by construction coincide with the Lovelock vacua solutions of $A d S_{d}$ type. As is well known at such critical points in the coupling space the corresponding $Q F T_{d-1}$ becomes conformal invariant and phase transitions of second or infinite order take place [31-33]. The different non-constant solutions $\sigma_{k j}=\sigma\left(l ; \sigma_{k}^{*}, \sigma_{j}^{*}\right)$ are directly related to the flat DWs solutions of the considered Quasi-Topological Gravity-matter models (2.1). As it is demonstrated in ref.[5], the near-boundary/horizon properties of such DW's, representing specific ( $a) A d S_{d}$ space-times, determine important characteristics of the Lovelock's vacua and of their dual $C F T_{d-1}$ 's, namely the "critical exponents" $s_{k}=-\frac{d \beta}{d \sigma}\left(\sigma_{k}\right)\left(\right.$ for $\left.W_{k} \neq 0\right)$ :

$$
\begin{gathered}
s_{a}=2(d-2) \frac{W_{a}^{\prime \prime}}{\kappa^{2} W_{a}}\left[1-\frac{2 \lambda L^{2}}{(d-2)^{2}} \kappa^{2} W_{a}^{2}-\frac{3 \mu L^{4}}{(d-2)^{4}} \kappa^{4} W_{a}^{4}\right]=2(d-2) \frac{W_{a}^{\prime \prime}}{\kappa^{2} W_{a}}\left(1-\frac{L_{+}^{2}}{L_{a}^{2}}\right)\left(1-\frac{L_{-}^{2}}{L_{a}^{2}}\right), \\
s_{\text {top }}^{ \pm}=-\frac{8}{(d-2)} W_{ \pm}^{\prime 2}\left[\lambda L^{2}+\frac{3 \mu L^{4}}{(d-2)^{2}} \kappa^{2} W_{ \pm}^{2}\right]=-\frac{4 L_{ \pm}^{2}}{(d-2)} W_{ \pm}^{\prime 2}\left[1-\frac{L_{ \pm}^{2}}{L_{ \pm}^{2}}\right]
\end{gathered}
$$

These new parameters characterize the near-boundary (and near-horizon) behaviours of the scalar field and of the scale factor:

$$
\sigma(y) \stackrel{y \rightarrow \infty}{\approx} \sigma_{k}^{*}+\text { const. } e^{-s_{k} \frac{y}{L_{k}}}, \quad e^{2 A(\sigma)} \sim\left(\sigma-\sigma_{k}\right)^{-\frac{2}{s_{k}}},
$$


which can be easily checked by taking appropriate limits of eqs. (2.2) with $W(\sigma)$ replaced by:

$$
W(\sigma) \approx W\left(\sigma_{k}^{*}\right)+\frac{W^{\prime}\left(\sigma_{k}^{*}\right)}{2}\left(\sigma-\sigma_{k}^{*}\right)^{2} .
$$

for each one of the physical vacua $W^{\prime}\left(\sigma_{k}^{*}\right)=0$. Observe that the vacua $\left(\sigma_{k}, L_{k}, s_{k}\right)$ with $s_{k}<0$ describe the horizons (i.e. we have that $e^{2 A} \rightarrow 0$ ), while for the vacuum with $s_{k}>0$ we find that $e^{2 A} \rightarrow \infty$, thus representing the $A d S_{d}$ boundary.

In the case of higher order $n_{k}>1$ zeros of the $\beta\left(\sigma_{k}\right)$-function, i.e. when also all its derivatives $\beta^{\left(n_{k}\right)}\left(\sigma_{k}\right)=0$ are vanishing, we find a new type of near-boundary behaviour of the matter field:

$$
\sigma(l) \stackrel{y \rightarrow \infty}{\approx} \sigma_{k}^{*}+\operatorname{const} .\left(\frac{y}{L_{k}}\right)^{\frac{1}{1-n_{k}}},
$$

Notice that such asymptotic values of the running coupling constant in the dual $Q F T_{d-1}$ models are specific for the neighbours of a "marginal" critical point $\sigma_{k}^{\text {marg }}$ of vanishing critical exponent $s_{k}^{\text {marg }}=0$, where an infinite order phase transition takes place.

2.2.2. CFT-data: BF unitarity conditions. The conditions of stability of the physical vacuum, expressed by $C_{0}\left(f_{k}\right)>0$, are known to be a part of the consistency requirements for the unitarity of the dual $C F T_{d-1}$ 's $[5,17]$. Let us remind the additional restrictions obtained by imposing the Breitenlohner-Freedman (BF) unitarity condition [29]:

$$
-\frac{(d-1)^{2}}{4 L_{k}^{2}} \leq m_{\sigma}^{2}\left(\sigma_{k}^{*}\right)=V^{\prime \prime}\left(\sigma_{k}^{*}\right)
$$

It assures the stability of the gravity-matter vacua $\left(\sigma_{k}^{*}, \Lambda_{\text {eff }}^{k}<0\right)$ with respect to the linear fluctuations of the scalar field. Taking into account (2.13), together with the definitions (2.9) of the critical exponents $s_{k}$, it is easy to derive the relation of the "vacua masses" $m_{\sigma}^{2}\left(\sigma_{k}^{*}\right)=V^{\prime \prime}\left(\sigma_{k}^{*}\right)$ of the scalar field to the anomalous dimensions $\Delta_{k}=d-1-s_{k}$ of the corresponding "dual" conformal fields $\Phi_{\sigma_{k}}\left(x_{i}\right)$ :

$$
m_{\sigma}^{2}\left(\sigma_{k}^{*}\right)=\frac{\kappa^{2} W_{k}^{2}}{(d-2)^{2}} s_{k}\left(s_{k}-d+1\right)=\frac{1}{L_{k}^{2}} s_{k}\left(s_{k}-d+1\right) .
$$

The above mass formulas are valid for all the Lovelock's vacua $\sigma_{A}^{*}$ - either the "physical" (or "ghost"-like), $\sigma_{k}^{*}$, or the topological, $\sigma_{\text {top }}^{ \pm}$, ones. They are obtained from eq. (2.3) by calculating the values of $V^{\prime \prime}(\sigma)$ at the corresponding vacuum and next by expressing them in terms of the critical exponents (2.9).

Notice that the BF-condition (2.13) is automatically satisfied for all the values of $s_{k}$. As one can see from Fig.(1), for each (positive or negative) value $m_{k}^{2} \neq-\frac{(d-1)^{2}}{4 L_{k}^{2}}$ of the mass, there exist always two different critical exponents $s_{k}^{ \pm}$, such that $s_{k}^{+}+s_{k}^{-}=d-1$. They provide two different asymptotic behaviours of the scalar field $\sigma(y)$, as we have explained above. The scaling dimensions of the corresponding $C F T_{d-1}$ fields $\Phi_{s_{k}^{ \pm}}\left(x_{i}\right)$ are given by:

$$
\Delta_{k}^{ \pm}=d-1-s_{k}^{ \pm}=\frac{d-1}{2} \pm \sqrt{\frac{(d-1)^{2}}{4}+m^{2}\left(\sigma_{k}^{*}\right) L_{k}^{2}}
$$


They are known to describe different states that give rise to different quantizations of the "boundary" $C F T_{d-1}$. As argued in ref. [30], only the field corresponding to $\Delta^{+}$(under certain restrictions) can drive the RG flow from one UV to the IR CFT's, thus defining a non-conformal perturbation of the $C F T_{U V}$ as in eq. (1.2). Instead, the conformal field with $\Delta^{-}$leads to spontaneous breaking of the conformal symmetry by introducing a new vacuum state and non-vanishing mean values $\left\langle\Phi_{s^{-}}(0)>\neq 0\right.$.

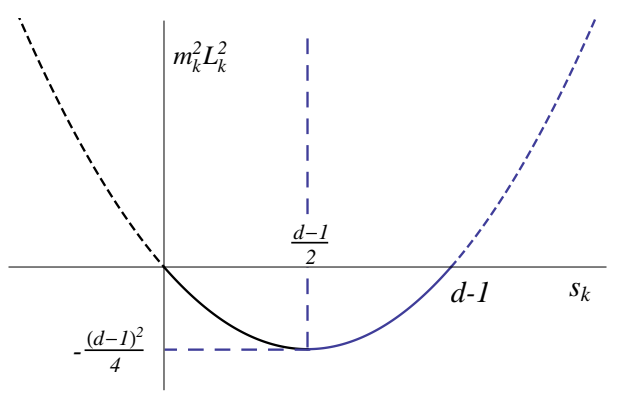

Figure 1. The $m_{k}^{2} L_{k}^{2}$ curve as a function of $s_{k}$ : 1.h.s. of the parabola plotted in black corresponds to $\Delta_{k}=\Delta_{k}^{+}$and its r.h.s.(in blue) represents $\Delta_{k}=\Delta_{k}^{-}$. On the dashed line $m_{k}^{2}>0$, while on the continuous one $m_{k}^{2}<0$.

As is well known one of the conditions for unitarity of these $C F T_{d-1}^{\prime} s$ is the positivity of the corresponding scaling dimensions $\Delta_{k}^{ \pm}>0$, which requires that ${ }^{10} s_{k}<d-1$. Since we are interested in the holographic description of the RG flows in such perturbed CFT's, we shall consider the case $\Delta_{k}^{+}$only. We further impose an important extra condition, namely that the operators $\Phi_{s^{+}}^{U V}\left(x_{i}\right)$ are relevant: $0<\Delta_{U V}^{+}<d-1$. Then, according to eq. (2.15) we obtain the following restriction:

$$
\frac{d-1}{2}<\Delta_{U V}^{+}<d-1 \quad \text { or equivalently } \quad 0<s_{U V}^{+}<\frac{d-1}{2},
$$

which ensures the existence UV/IR- RG flows in the dual unitary $Q F T_{d-1}$. Observe that the above conditions are satisfied only for negative values ${ }^{11}$ of the scalar field masses $m_{U V}^{2}$ :

$$
-\frac{(d-1)^{2}}{4} \leq m_{\sigma}^{2}\left(\sigma_{U V}^{*}\right) L_{U V}^{2}<0
$$

This stronger form of the BF-condition, together with the other physical restrictions on values of $A d S_{d}$ physical scales $L_{k}$, are responsible to qualitative changes in the shape of the matter potential, compared to the case when $s_{U V}>d-1$, as it is demonstrated in refs. [5, 73] (see also Sect.7.3 below).

2.2.3. CFT-data: OPE's structure constants. Let us remember that the conformal invariance [33, 39] determines the exact form of all the 2- and 3-point functions of the

\footnotetext{
10 the values of $s_{k}^{-}>d-1$ correspond to $\Delta_{k}^{-}<0$, thus giving rise to non-unitary CFT's.

11 it is worthwhile to mention that for positive masses $m_{U V}^{2}>0$ we have that $\Delta_{U V}^{+}>d-1$, i.e $s_{U V}^{+}<0$ is negative, and therefore the corresponding operators $\Phi_{\sigma_{k}}\left(x_{i}\right)$ are irrelevant.
} 
fields $\Phi_{\left(\Delta, j_{k}\right)}(\vec{x})$ of conformal (scaling) dimensions $\Delta_{\Phi}$ and spins $j_{k}$ in a given $C F T_{d-1}$. For example, in the case of scalar fields they are given by:

$$
\begin{aligned}
& <\Phi_{\Delta}\left(\overrightarrow{x_{1}}\right) \Phi_{\Delta}\left(\overrightarrow{x_{2}}\right) \Phi_{\Delta}\left(\overrightarrow{x_{3}}\right)>=C_{\Phi}^{(3)}\left(\left|\overrightarrow{x_{12}}\right|\left|\overrightarrow{x_{13}}\right|\left|\overrightarrow{x_{23}}\right|\right)^{-\Delta_{\Phi}}, \\
& <\Phi_{\Delta}\left(\overrightarrow{x_{1}}\right) \Phi_{\Delta}\left(\overrightarrow{x_{2}}\right)>=C_{\Phi}^{(2)}\left|\overrightarrow{x_{12}}\right|^{-2 \Delta_{\Phi}}
\end{aligned}
$$

In order to complete the description of the properties of the relevant operator $\Phi_{s^{+}}^{U V}\left(x_{i}\right)$ we need to know the value of its scaling dimension $\Delta_{U V}^{+}=d-1-s^{+}$, given by eq. (2.9), and the explicit form of the short-distance OPE's ${ }^{12}$ as well:

$$
\Phi_{s^{+}}(1) \Phi_{s^{+}}(2) \approx C_{\Phi}^{(2)}\left|x_{12}\right|^{2\left(s^{+}-d+1\right)}+C_{\Phi \Phi \Phi}\left|x_{12}\right|^{s^{+}-d+1} \Phi_{s^{+}}(2)+\ldots
$$

We have introduced the OPE's structure constant $C_{\Phi \Phi \Phi}=C_{\Phi}^{(3)} / C_{\Phi}^{(2)}$, which turns out to coincide with the ratio of the 2- and 3-point normalization's constants.

The standard perturbative QFT's calculations [12, 33, 44] based on the "perturbed CFT" $(p C F T)$ action (1.2), involving the exact form of the 2- and 3-point functions (2.17) and the most singular terms of the OPE (2.18), give the well known result for the corresponding perturbative $\beta$-function:

$$
\beta(\sigma) \approx-s^{+}\left(\sigma-\sigma_{U V}^{*}\right)+C_{\Delta}\left(\sigma-\sigma_{U V}^{*}\right)^{2}+\ldots, \quad C_{\Delta}=\frac{\pi^{(d-1) / 2}}{\Gamma((d-1) / 2)} C_{\Phi \Phi \Phi}
$$

Therefore the knowledge of the holographic $\beta$-function (2.8) allows to determine the exact value of the CFT's OPE structure constants ${ }^{13}$ in terms of the values of the superpotential and its derivatives at the corresponding UV vacuum $\sigma_{U V}$ :

$$
2 C_{\Phi \Phi \Phi} \frac{\pi^{(d-1) / 2}}{\Gamma((d-1) / 2)}=\beta^{\prime \prime}\left(\sigma_{U V}^{*}\right)=\frac{2(d-2) W_{U V}^{\prime \prime \prime}}{\kappa^{2} W_{U V}} C_{0}\left(W_{U V}\right) .
$$

This identification provides not only an important non-perturbative CFT-data concerning the OPE's of the dual fields $\Phi_{s^{+}}^{U V}\left(x_{i}\right)$, but it also imposes specific restrictions on the form of the superpotential $W(\sigma)$. Namely, in order to have consistent $p C F T$ 's defined by the action (1.2) the requirement $W_{U V}^{\prime \prime \prime} C_{0}\left(W_{U V}\right) \neq 0$ must be satisfied, i.e. in the case of polynomial superpotentials, $W$ should be at least cubic.

2.2.4 Conserved currents OPE's. As usually the set of conserved currents - $J_{k}(\vec{x})$ of dimension $\Delta_{J}=d-2, T_{i j}(\vec{x})$ of dimension $\Delta_{T}=d-1$, etc. - and their correlation functions play an important role in the definition of the corresponding conserved charges, of their algebra and of the physical states of an arbitrary $Q F T_{d-1}$. It is well known that, in the absence of additional (extra) symmetries, almost all the important parameters specifying each $C F T_{d-1}$ are realized in terms of the constants of the 2- and 3-point functions of their

\footnotetext{
${ }^{12}$ notice that this form of the OPE's is oversimplified by neglecting the contributions of the irrelevant operators and of the less singular relevant operators as well.

${ }^{13}$ these exact values of the structure constants have to be distinguished from the perturbative values

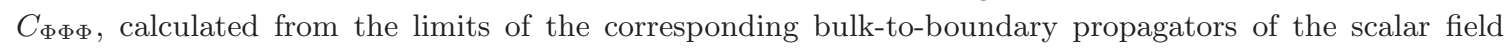
$\sigma\left(y, x_{i}\right)$, by applying the Witten's diagrams techniques [1].
} 
stress-tensors $T_{i j}\left(x_{k}\right)$ (with $T_{i}^{i}=0$ ). As in the case of the scalar field ${ }^{14}$ the corresponding 2-point function:

$$
\begin{aligned}
<T_{i j}\left(x_{k}\right) T_{p q}(0)> & =\frac{\pi^{(d-1) / 2}}{\Gamma((d-1) / 2)} \frac{c(d)}{x^{2(d-1)}} J_{i j, p q}(\vec{x}), \quad I_{i j}=\eta_{i j}-\frac{2 x_{i} x_{j}}{x^{2}} \\
J_{i j, p q}(\vec{x}) & =\frac{1}{2}\left[I_{i p}(\vec{x}) I_{j q}(\vec{x})+I_{i q}(\vec{x}) I_{j p}(\vec{x})\right]-\frac{\eta_{i j} \eta_{p q}}{d-1}
\end{aligned}
$$

contains only one parameter $c(d)$, while in the 3-point function:

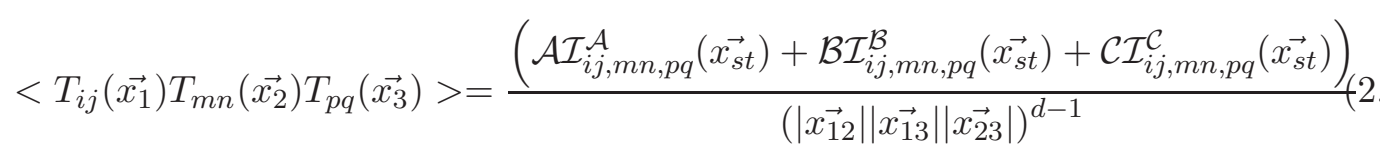

we need three constants $\mathcal{A}, \mathcal{B}$ and $\mathcal{C}$, representing the number of the different independent (conformal covariant) tensor structures $\mathcal{I}_{i j, m n, p q}^{\mathcal{K}}\left(\overrightarrow{x_{s t}}\right)$, that also satisfy the corresponding traceless and conservation $\partial^{i} T_{i j}(\vec{x})=0$ conditions $^{15}$. The conformal Ward identities further imply that the following relation between the constants of the $T_{i j}$ 's 2 - and 3 -point functions to take place [58]:

$$
c(d)=\frac{(d-2)(d+1) \mathcal{A}-2 \mathcal{B}-4 d \mathcal{C}}{d^{2}-1} .
$$

Similarly to the scalar fields OPE's (2.18), the the structure constants of the stress-tensor $T_{i j}(\vec{x}) T_{m n}(0)$ OPE's turns out to be simply related to the ratios of specific linear combinations of $\mathcal{A}, \mathcal{B}$ and $\mathcal{C}$ with the $c(d)$-central charge [48, 58].

\subsection{Weyl anomalies and central functions}

Let us remind that the higher $d \geq 4$ dimensional conformal $S O(d-1,2)$ symmetry is not restricting completely the $C F T_{d-1}$ dynamics [33,39]. Although it is again enough to determine all the 2- and 3-point functions (2.17) and (2.22), it is not sufficient to fix the form of the $n \geq 4$-point functions, which require the knowledge of the solutions of the conformal bootstrap equations [32]. The corresponding $C F T_{d-1}$ are characterized by: (1) the anomalous dimensions $\Delta_{k}=d-1-s_{k}$ and the spins $j_{a}$ of the conformal fields $\Phi_{\Delta, j_{a}}\left(x_{i}\right)$, defining certain unitary representations of the conformal group [39]; (2) the so called central charges $c_{n}, a, b_{l}$ and related to them coefficients - $\mathcal{A}, \mathcal{B}, \mathcal{C}$ - of the stress-tensor 3 -point function; and (3) the structure constants $C_{\Phi \Phi \Phi}$ of the OPE's of $\Phi_{k}\left(x_{i}\right)$ 's.

In order to complete the list of the remaining $C F T_{d-1}$ data that can be calculated by the RG and/or other perturbative methods, we next consider the definitions of a part of the central charges, namely $c=c_{2}$ and $a$. For odd values of $d$, i.e. for even dimensional $C F T_{d-1}$ 's with $d=2 n+1$, the above central charges appear as the (coupling dependent) coefficients in the Weyl (trace) anomaly expression [40, 56]:

$$
\left.<T_{i}^{i}>\sim \sum c_{n}(d, \sigma) I_{n}(d)-2(-1)^{(d-1) / 2} a(d, \sigma) E_{d-1}+b_{l} \text { (current's anomalies) }\right)_{l},(2
$$

\footnotetext{
14 in fact the explicit form of all the 2- and 3- point functions of fields of arbitrary Lorentz spins is well known [39, 51, 52], involving an increasing number of independent tensor structures determined by the conditions of conformal symmetry.

15 we are omitting the explicit forms of these tensor structures [58], since they are irrelevant for the problems discussed in the present paper.
} 
where $I_{n}$ are the invariants constructed from up to $n=(d-1) / 2$ Weyl tensors $W_{i j k l}$ in $(d-1)$-dimensions and its covariant derivatives as for example $I_{2}(d=5)=W_{i j k l} W^{i j k l}$ and $E_{d-1}$ is the corresponding Euler invariant. The standard methods of the perturbative $Q F T_{d-1}$ calculations on arbitrary curved space background $[40,41,59]$ provide the explicit form of the gravitational part of these anomalies ${ }^{16}$ together with the one- and two-loop values of the anomaly coefficients $c$ and $a$ for considerably large family of $C F T_{4}$ 's. One representative example is given by the $L_{i n t}=\frac{g_{0}}{16} \phi^{4}$-model in four dimensions[49]:

$$
c\left(g_{0}\right) \approx 1-\frac{5 g_{0}^{2}}{36(4 \pi)^{4}}+\ldots, \quad a\left(g_{0}\right) \approx 1+\frac{85}{288}\left(\frac{g_{0}}{(4 \pi)^{2}}\right)^{4}+. .
$$

The exact values of $a$ and $c$ are known for the extended $\mathcal{N}$-supersymmetric $S U\left(N_{c}\right)$ gauge theories and in particular for the $\mathcal{N}=4$ SUSY Yang-Mills they do coincide: $c=a=\frac{\left(N_{c}^{2}-1\right)}{4}$, see for example refs.[42, 43, 51, 53].

2.3.1. The c-central charge. An equivalent definition of the central charge $c_{2}(d)=$ $c(d)$, valid for arbitrary dimensions $\mathrm{d}$, is as the properly normalized coefficient of the 2point function of the stress-tensor (2.21). One important consequence is that $c(d)$ in fact determines the norms of the stress-tensor's "states" $T_{i j}(0) \mid 0>$ and therefore it must be positive $c(d)>0$ as a part ${ }^{17}$ of the unitarity conditions for such $C F T_{d-1}$. For each given even-dimensional $d-1=2 n$ (super)conformal model containing few massless free fields: $n_{s}$ scalars, $n_{v}$ vectors and $n_{f}$ spinor fields it is relatively easy to calculate from eq. (2.21) the exact value of the $c$ - central charge $[46,51]$ :

$$
c_{\text {free }}(2 n)=n_{s}+2^{n-1}(2 n-1) n_{f}+\frac{(2 n) !}{2((n-1) !)^{2}} n_{v} \neq a_{\text {free }}(2 n) .
$$

Notice that the interaction is modifying these values as in the case $n_{s}=1$ and $n_{f}=0=n_{v}$ of the above mentioned $\phi^{4}$ model.

We next briefly recall the holographic derivation of eq. (2.21). The starting point is the effective action of the cubic QT Gravity considered as a functional of the arbitrary "boundary metrics" $h_{i j}^{0}\left(x_{i}\right)=h_{i j}\left(y \rightarrow \infty, x_{i}\right)$, calculated in the linear approximation $g_{\mu \nu}\left(y, x_{i}\right)=g_{\mu \nu}^{v a c}(y)+\kappa h_{\mu \nu}\left(y, x_{i}\right)$ around a given $A d S_{d}$ vacuum $\left(\sigma_{k}^{*}, L_{k}\right)[9,17]$ :

$$
S_{g b l}\left(h_{i j}^{0}\right)=\frac{\pi^{(d-1) / 2}}{\Gamma((d-1) / 2)} c_{Q T}\left(f_{k}\right) \int d^{d-1} x_{1} d^{d-1} x_{2} h_{i j}^{0}\left(x_{1}\right) \frac{J^{i j, p q}\left(\vec{x}_{12}\right)}{\left|\vec{x}_{12}\right|^{2 d}} h_{p q}^{0}\left(\vec{x}_{2}\right)
$$

with $c_{Q T}\left(f_{k}\right)=C_{0}\left(f_{k}\right)\left(\frac{L_{k}}{l_{p l}}\right)^{d-2}$. According to the $A d S_{d} / C F T_{d-1}$ correspondence [1], in the limit of large $\left(\frac{L_{k}}{l_{p l}}\right)^{d-2} \approx N_{c}^{2} \gg 1$, it can be considered as a generating functional of the 2-point correlation function of the stress-tensor of the dual $C F T_{d-1}$ :

$$
\left.\frac{1}{2} \frac{\delta^{2} S_{g b l}\left(h_{i j}^{0}\right)}{\delta h_{i j}^{0}(x) \delta h_{p q}^{0}(y)} \equiv<T^{i j}(\vec{x}) T^{p q}(\vec{y})\right)>_{C F T},
$$

\footnotetext{
${ }^{16}$ related at the lowest order to the corresponding $R^{2}$-type counter-terms

${ }^{17}$ together with certain restrictions on the conformal dimensions $\Delta_{k} \geq 0$
} 
This identification provides an important non-perturbative information about the strongcoupling limit of $c(d)$-central charge:

$$
c_{Q T}\left(d ; L_{k}, \lambda, \mu\right)=\left(\frac{L_{k}}{l_{p l}}\right)^{d-2}\left(1-2 \lambda f_{k}-3 \mu f_{k}^{2}\right), \quad f_{k}=\frac{\kappa^{2} L^{2} W_{k}^{2}}{(d-2)^{2}}=\frac{L^{2}}{L_{k}^{2}} .
$$

Notice that the new QT gravity contributions can be considered as the next-to-leading order term in the $l_{p l} / L_{k}$ expansion of $c_{Q T}(\lambda, \mu)$ to the (leading) EH-order ${ }^{18}$, i.e. $c_{E H} \approx$ $\left(\frac{L_{k}}{l_{p l}}\right)^{d-2}$. The fact that the stability condition selecting the physical vacua $C_{0}\left(f_{k}\right)>0$ of ddimensional extended cubic Lovelock Gravity (2.1), discussed in Sect.2.2., is responsible for the unitarity of the dual $C F T_{d-1}$ 's, is an example of the holographic relationship between the causality (and stability) of the (semi)classical Gravitational models and the unitarity of their dual $Q F T_{d-1}$ 's.

The above expression (2.26) for the UV and IR values of the $c$-central charge (for $\left.L_{k}=L_{U V / I R}\right)$, together with the well known form [3] of the coinciding $c_{E H}=a_{E H^{-c e n t r a l}}$ functions in the case of EH- $A d S_{d}$ gravity-matter models, suggest the following definition of the cubic QT Gravity $c$-central function [9]:

$$
c(d, \sigma)=\frac{(d-2)^{d-2}}{\left(-l_{p l} \kappa W\right)^{d-2}}\left(1-2 \lambda \frac{\kappa^{2} L^{2} W^{2}(\sigma)}{(d-2)^{2}}-3 \mu \frac{\kappa^{4} L^{4} W^{4}(\sigma)}{(d-2)^{4}}\right),
$$

It is worthwhile to mention its relation to the holographic $\beta$-function (2.8):

$$
\beta(d, \sigma)=-2(d-2) \frac{W^{\prime}}{\kappa^{2} W} C_{0}(\sigma)=-c(d, \sigma) \frac{d g^{-1}(\sigma)}{d \sigma}, \quad g(\sigma)=\frac{\kappa^{2}(d-2)^{d-2}}{2\left(-l_{p l} \kappa W\right)^{d-2}},
$$

where the function $g(\sigma)$ represents the one-dimensional analogue of the Zamolodchikov's coupling space metrics $[12,43,57]$ and it turns out to coincide with the constant of $\Phi_{\sigma}$ 2-point function $g=C_{\Phi}^{(2)}$.

An important comment about the relations between the UV and IR values of the $c(d)$ central charge is now in order. In two dimensional CFT's (i.e.for $d=3$ ) it represents the central charge of the Virasoro algebra [45] and according to the Zamolodchikov's $c$-theorem [12] for a large class of unitary perturbed $C F T_{2}$ 's, admitting peturbative UV and IR critical points, we have $c_{U V}>c_{I R}$ and the corresponding $c(d=3, \sigma)$-function is monotonically decreasing during the massless RG flow. In higher dimensions, according to the Cardy's conjecture[13], a similar role is played by the $a(d>3)$-central charge, which satisfies a d-dimensional " $a$-theorem": $a_{U V} \geq a_{I R} \geq 0$. On the other hand, for the corresponding $c(d>3)$-central function there exist representative QFT's examples demonstrating that one can observe three different behaviours along the $\mathrm{RG}$ flow: decreasing $\frac{d c}{d l}<0$, increasing $\frac{d c}{d l}>0$ or non-monotonic $c(l)$-function ${ }^{19}$. Although in the few known cases such behaviour is related to the particular restrictions on the values of the ratio $N_{f} / N_{c}$ of numbers of the flavors and colors in the corresponding $S U\left(N_{c}\right) Q C D_{4}$ (see for example ref. [46]), a clear field-theoretical explanation of these different RG evolutions of the $c$-central charge is still

\footnotetext{
${ }^{18}$ in the dual $S U\left(N_{c}\right)$ CFT's with $N_{c} \gg 1$ it can be considered as representing the next order in $1 / N_{c}$ expansion.

${ }^{19}$ see for example refs. $[43,46]$ for the systematic discussion of such models
} 
missing. In order to find the corresponding holographic explanation, the RG flows in certain (super)conformal models with non-equal central charges $c \neq a$ have to be studied. Their holographic description is known to require the explicit form of certain domain walls in the GB or/and cubic QTG extensions of the EH-gravity [5, 7, 8]. This problem is addressed in Sect.3. below in the frameworks of our proof of the cubic Quasi-Topological Gravity holographic " $a / c$-Theorems".

2.3.2. The a-central charge. The coefficient in front of the Euler invariant $E_{d-1}$ in the eq. (2.24), known as $a$-central charge, possesses few others holographic and purely $Q F T_{d-1}$ 's definitions and physical interpretations. Its $C F T$ 's origin is as an independent parameter that, together with the c-central charge (2.23) and the energy fluxes parameter $t_{4},{ }^{20}$ determines the coefficients $\mathcal{A}, \mathcal{B}$ and $\mathcal{C}$ in the 3 -point stress-tensor function (2.22). For $d=5$ these relations have the form $[7,9]$ :

$$
a=\frac{13 \mathcal{A}-2 \mathcal{B}-40 \mathcal{C}}{72}, \quad t_{4}=-\frac{15(17 \mathcal{A}+32 \mathcal{B}-80 \mathcal{C})}{4(9 \mathcal{A}-\mathcal{B}-10 \mathcal{C})}
$$

Equivalently it takes part of the definitions of the "structure constants" of particular channels in the $T_{i j}(x) T_{p q}(0) \mid 0>$ OPE's (see for example Sect.6 of ref. [48]). There exist many CFT's examples for which the perturbative UV/IR values of the $a$-central charge have been calculated $[42,51,53,59]$. Differently from the $c(d>3)$-charges they are always decreasing $a_{U V}>a_{I R}$ and their free field limits (when appropriately normalized) reproduce the effective number of the massless degrees of freedom.

We find however that similarly to the well known two dimensional case [12], the simplest and the most useful definition of the $a$-central function (for all the values of $d$ ) is as the pre-potential

$$
\beta(\sigma)=\frac{1}{g(\sigma)} \frac{d a(\sigma)}{d \sigma}
$$

for the $\beta$-function of the corresponding dual $Q F T_{d-1}$ model. Starting from the explicit form (2.8) of the $\beta(d, \sigma)$ in terms of the superpotential $\mathrm{W}$, and following the original two dimensional proof [12], we find that the $a$-function is given by:

$$
a(d, \sigma)=\frac{(d-2)^{d-2}}{\left(-l_{p l} \kappa W\right)^{d-2}}\left(1-2 \lambda\left(\frac{d-2}{d-4}\right) \frac{\kappa^{2} L^{2} W^{2}(\sigma)}{(d-2)^{2}}-3 \mu\left(\frac{d-2}{d-6}\right) \frac{\kappa^{4} L^{4} W^{4}(\sigma)}{(d-2)^{4}}\right) 2
$$

up to an additive constant $a_{0}$. Observe that it indeed coincides with the standard holographic definition $[3,7,9]$ obtained from the corresponding Quasi-topological Gravity DW's equations, that leads to monotonically decreasing $a$-function, when the "null energy" condition for the scalar matter is fulfilled. The above relation (2.30) between the $\beta$ and the $a$-functions has an important consequence:

$$
\frac{d a}{d l}=-\beta^{2}(\sigma) g(\sigma)<0
$$

\footnotetext{
${ }^{20}$ Notice that $t_{4}$ is zero for arbitrary $\mathcal{N}=1,2,4$ SUSY CFT's as well as in all the $C F T_{d-1}$ duals of the GB Gravity models [7, 9]; see Sect.6 below for further details.
} 
that provides the simplest QFT's proof of the monotonic decreasing of the $a$-central function (2.31) with the RG scale $l$ for positive definite "metric" $g(\sigma)>0$.

Again as in eq. (2.26) above, the "1-d Zamolodchikov's coupling space metric" $g(\sigma)^{21}$ is involved in the relations between $\beta$ and the $a$-function derivatives. Its presence reflects the properties of the particular holographic "renormalizaton scheme" (called Wilsonian in Sect.2. of ref. [57]), which turns out to be different from the Zamodchikov's one (used in his proof of $2 d c$-theorem), that leads to constant metric and "gradient flows" [12]. As is well known the RG transformation $d \tilde{\sigma}=\sqrt{g(\sigma)} d \sigma$ interpolates between the holographic and the Zamolodchikov's (constant metric) RG scheme [43, 47, 57], thus changing the form of the $\beta, a$, and $c$-functions, but leaving the values of the UV critical exponents and also the form of $\frac{d a}{d l}(2.32)$ unchanged. Let us also mention the importance of the "geometric" analogue $W(\sigma)<0$ of the $g>0$ condition, we are currently requiring [5] in order to ensure that the DW's $(a) A d S_{d}$ geometries describing the holographic RG flows belong to the family of standard asymptotically $A d S_{d}$ space-times having one (UV-type) boundary at $y \rightarrow \infty$ and one horizon (of IR type) at $y \rightarrow-\infty{ }^{22}$.

It is worthwhile to mention that the $a$-Theorem statement is in fact equivalent of the $Q F T_{d-1}$ unitarity condition of positive definiteness of the norm of states created by the trace $\Theta\left(\sigma, x_{i}\right)=\beta(\sigma) \Phi_{\sigma}\left(x_{i}\right)$ of the stress-tensor, i.e.

$$
<\Theta(\vec{x}) \Theta(0)>=\beta^{2}(\sigma)<\Phi_{\Delta}(\vec{x}) \Phi_{\Delta}(0)>=\beta^{2} g(\sigma)|\vec{x}|^{-2 \Delta_{\Phi}}>0 .
$$

It requires that $\beta^{2} g>0$ or equivalently $\frac{d a}{d l}<0$, i.e. $a$-central function must be decreasing during the massless RG flow.

Taking into account eqs. (2.30) and (2.28), we next derive the following relation between the $a, g$ and $c$-functions:

$$
c(\sigma)=g \frac{d a}{d g},
$$

demonstrating that the $a(W)$-central function, when considered as a function of $g(\sigma)$, appears to be a pre-potential for the $c$-function as well. This fact provides an equivalent form of the $a$-Theorem ${ }^{23}$ :

$$
\Delta a \equiv a_{U V}-a_{I R}=-\int_{\sigma_{U V}}^{\sigma_{I R}} \beta(\sigma) g(\sigma) d \sigma=-\int_{g_{U V}}^{g_{I R}} \frac{c(g)}{g} d g>0
$$

relating the $R G$ flux of the $a$-function $\Delta a$ to the invariant area of the $\beta(\sigma)$-function, that now can be replaced by $c / g$. Notice that for vanishing Lovelock couplings $\lambda=0=\mu$, i.e for the $Q F T_{d-1}$ 's duals to $E H$-gravity-matter models, the $a$ and $c$-functions are proportional of the "couplings metric":

$$
\left.a_{E H}(W)=c_{E H}(W)=2 g(W)\right) / \kappa^{2},
$$

\footnotetext{
${ }^{21}$ although it is not quite appropriate to introduce metrics in one-dimensional spaces, this terminology is frequently used (see for example refs. $[43,57]$ ) following the analogy with the sigma-model like metrics $G_{a b}\left(\sigma_{a}\right) d \sigma^{a} d \sigma^{b}$ in the case of many couplings $\sigma_{b}, a, b=1,2, \ldots, N$

${ }^{22}$ thus avoiding $(a) A d S_{d}$ spaces of two boundaries or two horizons that occur when $W$ has zeroes as in $d=3$ examples studied in refs. [6, 34, 37, 38] and also for the Janus-type DWs of ref. [3].

${ }^{23}$ advocated by D.Anselmi in ref. [46, 47]
} 
which considerably simplifies all the relations we have derived above.

Two comments concerning the QFT's and gravitational meaning of the $a$-central function are now in order:

- The important physical identification $[9,65]$ of the $a$-central charges (valid for both even and odd dimensions) as representing the universal coefficient in the leading contribution to the entanglement entropy in the dual CFT's(in specific boundary geometries) have been recently confirmed by numerous CFT's and holographic calculations [62]. The explicit DW's solutions and related to them Holographic RG flows in the dual QFT's provide an efficient tool for studying the off-critical properties of the entanglement entropy.

- The relation between the UV and IR values of the $a$-central charge and the tensions of the cibic QT Gravity DW's is based on the following suggestive total derivative form of QTG action (2.1):

$$
S_{G B L}^{\mathrm{eff}}=-\frac{2}{l_{p l}(d-2)^{d-1}} \int d^{d-1} x d y \frac{d}{d y}\left[e^{(d-1) A(y)}\left(-l_{p l} \kappa W\right)^{d-1} a(W)\right],
$$

derived in ref. [5]. Taking into account the appropriate boundary terms [60,61] needed for the consistent definition of the QTG action and dividing by the covariant volume, one can easily calculate by the Brown-York method the corresponding DW's tensions ${ }^{24}$ in the terms of the $a$-central charges $a_{U V}$ and $a_{I R}$ and of the values of the coupling space metrics $g(U V / I R) \sim f^{\frac{2-d}{2}}(U V / I R)$.

\section{Holographic $a / c$-Theorems}

The critical behaviour of each $Q F T_{d-1}$, dual to the Quasi-Topological Gravity (QTG) coupled to scalar matter (2.1), is described by a set of distinct CFT's, characterized by their central charges $c_{k}$ and $a_{k}$, the sign and the values of the critical exponents $s_{k}$, the corresponding structure constants $C_{\Phi \Phi \Phi}$ and and by $\mathcal{A}, \mathcal{B}$ and $\mathcal{C}$. By construction they represent the physical and topological $Q T G$ 's vacua $\left(L_{k}: s_{k}, c_{k}, a_{k}, \mathcal{A}_{k}, \mathcal{B}_{k}, \mathcal{C}_{k}\right)$. The particular features of the "holographic RG evolution" of all this $C F T_{d-1}$-data between two consecutive critical points $\sigma_{k}$ and $\sigma_{k+1}$ is geometrically described by the corresponding $D W_{k, k+1}=D W\left(\sigma_{k}, \sigma_{k+1}\right)$ QTG domain wall, separating these vacua.

An important question to be answered concerns the specific restrictions we have to impose on the Lovelock couplings $\lambda$ and $\mu$ and on the shape of the superpotential $W(\sigma)$ in order to define a physically consistent 25 "holographic" $Q F T_{d-1}$. A part of these consistency conditions, related to the critical CFT-data as for example $c>0, a>0, \Delta_{\Phi}>0$ and $0<s_{U V}^{k}<(d-1) / 2$, have been already studied in Sect.2. above (see also refs. $[5,9,17]$ ).

\subsection{On the content of $a / c$ - Theorems}

This section is devoted to the problem of the additional off-critical requirements on the corresponding $a(l), c(l)$ and $g(l)$-functions, that allow us to extend the unitarity consistency

\footnotetext{
${ }^{24}$ see for example ref.[6,38] for the details concerning the simplest $d=3$ case.

${ }^{25}$ since the $\mathcal{N}=1$ and $\mathcal{N}=0$ (super-symmetric) $S U\left(N_{c}\right)$ gauge theories have not available strong-coupling $Q F T$ description, the consistency requirements on the corresponding holographic $\beta, a$ and $c$-functions are crucial for the independent holographic definition of such QFT's.
} 
of the $C F T_{d-1}(U V / I R)$ 's to the massless phases of the conjectured dual non-conformal $Q F T_{d-1}$. This set of conditions, known as "a/c-Theorems" of Zamolodchikov's and Cardy's $[12,13]$, have been intensively studied by the standard Wilson Renormalization Group and by perturbative field-theoretical methods as well $[50,51,54,55]$. Their holographic versions $[3,4]$, originally formulated for certain special $c=a \mathcal{N}=4$ super-symmetric $Q F T_{4}$ duals to $d=5$ Einstein (super)Gravity coupled to matter, have been recently extended to the case $c \neq a$ of most general (non-supersymmetric) $Q F T_{d-1}$ 's [9, 28] involving appropriate "higher derivatives" generalizations of the EH-gravity. As we have mentioned in Sect.2.3 above, the proof of the holographic EH-, GB- and extended Lovelock's a-theorems ${ }^{26}$ is almost straightforward and valid for all the matter stress-tensors that satisfy the null energy condition. The only important restriction turns out to be the "positive definiteness" of the Zamolodchikov's couplings space metric $g(\sigma)>0$, i.e. $W(\sigma)<0$. Another natural causality/stability/unitarity requirement is given by the positivity of the physical UV and IR $c$ - and $a$ - central charges and of the corresponding $c$ - and $a$ - central functions: $c(\sigma) \geq 0$ and $a(\sigma) \geq 0$, as reviewed in Sect.2. above ${ }^{27}$. The complete description of the Holographic $\mathrm{RG}$ flows and of the specific features of the physically consistent dual $Q F T_{d-1}$ 's requires more detailed study of the analytic properties of these central functions.

One of the advantages of the superpotential method is that it allows to explicitly realize the $a, c$ and $\beta$-functions in terms of $W(\sigma)$. Together with the running coupling $\sigma(l)$, obtained from the RG eq. (2.8), we have all the ingredients necessary to establish the dependence of the desired analytic properties of $a$ - and $c$-functions on the Lovelock couplings and on the $W$ 's parameters. We begin with few definitions (valid for $d>4$ and $d \neq 6)$ :

$$
\begin{aligned}
c(\sigma) & =\left(\frac{d-2}{-l_{p l} \kappa W}\right)^{d-2}\left(1-\frac{1}{f_{+}^{t o p}} \frac{L^{2} \kappa^{2} W^{2}}{(d-2)^{2}}\right)\left(1-\frac{1}{f_{-}^{t o p}} \frac{L^{2} \kappa^{2} W^{2}}{(d-2)^{2}}\right), \\
a(\sigma) & =\left(\frac{d-2}{-l_{p l} \kappa W}\right)^{d-2}\left(1-\frac{1}{f_{+}^{a}} \frac{L^{2} \kappa^{2} W^{2}}{(d-2)^{2}}\right)\left(1-\frac{1}{f_{-}^{a}} \frac{L^{2} \kappa^{2} W^{2}}{(d-2)^{2}}\right), \\
\frac{d c}{d l} & =-\frac{2(d-2)^{d}}{\kappa^{2} l_{p l}^{d-2}}\left(\frac{W^{\prime}(\sigma)}{W(\sigma)}\right)^{2} c(l)\left(1-\frac{1}{f_{+}^{c^{\prime}}} \frac{L^{2} \kappa^{2} W^{2}}{(d-2)^{2}}\right)\left(1-\frac{1}{f_{-}^{c^{\prime}}} \frac{L^{2} \kappa^{2} W^{2}}{(d-2)^{2}}\right),
\end{aligned}
$$

We have introduced together with the topological scales $\left(L_{o, t o p}^{2}\right)_{ \pm}=\left(L_{\text {top }}^{2}\right)_{ \pm} f_{ \pm}^{\text {top }}$ (see sect.2.1.) the following two intermediate scales $\left(L_{a}^{2}\right)_{ \pm}$and $\left(L_{c^{\prime}}^{2}\right)_{ \pm}$:

$$
\begin{aligned}
f_{ \pm}^{t o p} & \equiv \frac{L^{2}}{\left(L_{t o p}^{2}\right)_{ \pm}}=-\frac{1}{3 \mu}\left(\lambda \mp \sqrt{\lambda^{2}+3 \mu}\right) \\
f_{ \pm}^{a} & \equiv \frac{L^{2}}{\left(L_{a}^{2}\right)_{ \pm}}=-\frac{1}{3 \mu}\left(\frac{d-6}{d-4}\right)\left(\lambda \mp \sqrt{\lambda^{2}+3 \mu \frac{(d-4)^{2}}{(d-6)(d-2)}}\right) \\
f_{ \pm}^{c^{\prime}} & \equiv \frac{L^{2}}{\left(L_{c^{\prime}}^{2}\right)_{ \pm}}=-\frac{1}{3 \mu}\left(\frac{d-4}{d-6}\right)\left(\lambda \mp \sqrt{\lambda^{2}+3 \mu \frac{(d-6)(d-2)}{(d-4)^{2}}}\right)
\end{aligned}
$$

\footnotetext{
${ }^{26}$ i.e. the $d>3$ analogues of the Zamolodchikov's c-theorem

${ }^{27}$ the list of the papers devoted to the investigations of these two conditions in different GB- and Lovelockholographic models and especially of these ones on the physically allowed a-values is extensive [7, 8, 10, 11].
} 
Notice that $f_{ \pm}^{\eta}$ (where $\eta=t o p, a, c^{\prime}$ ) are not always real (and positive) numbers and depending on the signs and values of the $\lambda$ and $\mu$ (and on the value of $d$ ) they might also represent the two complex conjugate solutions $f_{+}^{\eta}=\left(f_{-}^{\eta}\right)^{*}$ of the corresponding quadratic equations.

With the explicit form (3.2) of the $f_{ \pm}^{\eta}$ at hand, and the conditions $c_{U V / I R}>0$ and $0<\Delta_{U V}^{+}<d-1$ already established in ref. [5], we are going to derive the remaining restrictions on $\lambda, \mu$ and $f(\sigma)=L^{2} \kappa^{2} W^{2}(\sigma) /(d-2)^{2}$ such that one of the following three versions of the $a / c$-Theorem are satisfied:

(1) Standard a/c-Theorem:

$$
c>0 \quad, \quad a>0 \quad, \quad \frac{d a}{d l}<0 \quad, \quad \frac{d c}{d l}<0
$$

i.e. both $a$ - and $c$-central functions are positive and monotonically decreasing in a given massless phase of the dual $Q F T_{d-1}$ between two critical points $\sigma_{U V}$ and $\sigma_{I R}{ }^{28}$. It represents a natural $c \neq a$ extension of the standard holographic $a=c$-theorem $[3,4,9]$.

(2) Modified c-Theorems:

$$
c>0 \quad a>0 \quad \frac{d a}{d l}<0 \quad \frac{d c}{d l}>0
$$

i.e. $a(l)$ is decreasing as always, but now $c(l)$ is monotonically increasing; or else we can have

$$
c>0 \quad a>0 \quad \frac{d a}{d l}<0 \quad \text { and } \quad \frac{d c}{d l} \quad \text { changing sign }
$$

i.e. the $c(l)$-central function is non-monotonic, due to the existence of a critical scale $l_{c r}^{c}=\left(L_{c^{\prime}}^{2}\right)_{ \pm}$such that $\frac{d c}{d l}\left(l_{c r}^{c}\right)=0$ (and critical coupling $\sigma_{U V}<\sigma\left(l_{c r}\right)<\sigma_{I R}$ ), where the $c$-derivative changes its sign.

(3) a-Weaken Theorems: The conditions of this weak form of the theorem are the same as in (1),(2a) and (2b) above, except that now the a-function can change its sign within the $\left(\sigma_{U V}, \sigma_{I R}\right)$ interval. This leads to the existence of another critical scale $l_{c r}^{a}=\left(L_{a}^{2}\right)_{ \pm}$ (and mass $M_{c r}^{2} \sim 1 / L_{a}^{2}$ ) defined by the a-function zeros $a\left(l_{c r}\right)=0$. In fact such RG flows are not massless anymore, due to the critical mass scale up to where we have a consistent (causal and unitary) $Q F T_{d-1}$.

\subsection{On the methods of proof}

Similarly to the derivation of the restrictions on the $f_{k}$ (and $h_{k}$ ) in Sect.2.1.(see ref. [5]) that lead to stable physical vacua in the different domains of admissible values of $\lambda$ and $\mu$, it is convenient to keep our choose of the fundamental scale as $L^{2}=L_{0+}^{2}\left(\mu_{+}\right)=L_{0-}^{2}\left(\mu_{-}\right)$. Let us remind that this fact, together with the existence of topological vacua $c\left(f_{ \pm}^{\text {top }}\right)=0$, determines two distinct gravitational models (of equal fundamental scales): $\left(\lambda, \mu_{ \pm}\right)$characterized by the particular form of the Lovelock coupling $\mu$ as a function of $\lambda$ and of the corresponding $f_{ \pm}^{\eta}\left(\lambda, \mu_{ \pm}\right) \equiv f_{ \pm}^{\eta}(\lambda)$ as well, see for example eqs. (2.6) and (2.7).

\footnotetext{
${ }^{28}$ defined as two consecutive zeros of the $\beta$-function (2.8)
} 
The crucial step in the proof of the theorem is to establish which of the $f_{ \pm}^{\eta}(\lambda)$ 's are real and positives, how they are ordered and finally, the dependence of this ordering on the values of $-\infty<\lambda<1 / 3$. In fact in most of the cases it is enough to know the biggest and the smallest positives $f_{ \pm}^{\eta}(\lambda)$ 's. Given the explicit form (3.2) of $f^{\eta}$, it is not difficult to find the conditions that ensure their reality, i.e. to derive the corresponding restrictions on $\lambda$ and $\mu$ such that the expressions under the square roots are positive, namely:

$$
\lambda^{2}-3 \mu X_{\eta}(d) \geq 0, \quad X_{\text {top }}=-1, \quad X_{a}=-\frac{(d-4)^{2}}{(d-2)(d-6)}, \quad X_{c^{\prime}}=-\frac{(d-2)(d-6)}{(d-4)^{2}}(3.6)
$$

In order to find the reality restrictions for the $\mu_{ \pm}$models, we substitute the $\mu_{ \pm}$values (2.5) in the eqs. $\lambda^{2}-3 \mu X_{\eta}(d)=0$ that lead to the following quadratic equations for the "critical" values $\lambda_{\eta}^{(1,2)}$, where $f_{+}^{\eta}(\lambda)=f_{-}^{\eta}(\lambda)$ :

$$
\frac{81}{4} X_{\eta}^{2} \lambda^{2}+\left(\frac{81}{2} X_{\eta}+27\right) \lambda-9\left(\frac{3}{4}+X_{\eta}\right)=0
$$

Their solutions have the following simple form:

$$
\lambda_{\eta}^{(1,2)}=-\frac{2}{3 X_{\eta}^{2}}\left(\frac{3}{2} X_{\eta}+1 \mp\left(1+X_{\eta}\right)^{3 / 2}\right),
$$

or more explicitly

$$
\begin{gathered}
\lambda_{\text {top }}^{ \pm}=\frac{1}{3}, \quad \lambda_{\mu_{+}}^{a}=\frac{d(d-4)}{3(d-2)^{2}}<\frac{1}{3}, \quad \lambda_{\mu_{-}}^{a}=\frac{1}{3}-\frac{4}{3(d-6)^{2}}<\lambda_{\mu_{+}}^{a} \\
\lambda_{\mu_{ \pm}}^{c^{\prime}}=\frac{(d-6)(d-2)}{3(d-4)^{4}}\left(24+d(d-8) \mp \frac{16}{\sqrt{-(d-6)(d-2)}}\right) \in \mathbb{C}, d \geq 7,
\end{gathered}
$$

Together with the well known restriction $\lambda \leq \lambda_{\text {top }}^{ \pm}=1 / 3$ providing the $f_{\text {top }}^{ \pm}$reality, we realize that the $f_{a}^{ \pm}\left(\lambda ; \mu_{+}\right)$are real for $\lambda<\lambda_{\mu_{+}}^{a}$ and $f_{a}^{ \pm}\left(\lambda ; \mu_{-}\right)$- when $\lambda<\lambda_{\mu_{-}}^{a}$. For $d>6$ both $f_{c^{\prime}}^{ \pm}\left(\mu_{+}\right)$and $f_{c^{\prime}}^{ \pm}\left(\mu_{-}\right)$are always real, while for $d=5$ this condition is satisfied for $\lambda \in(-9+16 / \sqrt{3}, 1 / 3)$ only.

Although not always all of the $f_{ \pm}^{\eta}$ 's are real and positive, the factorization (3.1) of the three quadratic forms in simple multipliers (involving their roots) is the simplest and very well known method of studying the conditions under which they can have definite signs, say $c>0, a \geq 0$ and $\frac{d c}{d l} \leq 0$. In the case of the standard $a / c$ - Theorem, we have therefore to find the conditions such that:

$$
\left(1-\frac{f(\sigma)}{f_{+}^{\eta}}\right)\left(1-\frac{f(\sigma)}{f_{-}^{\eta}}\right), \quad \text { with } \quad f(\sigma) \equiv \frac{L^{2} \kappa^{2} W^{2}(\sigma)}{(d-2)^{2}}
$$

is positive for all the $\eta=t o p, a, c^{\prime}$ 's. There are four distinct cases for each one of the $\eta$ 's: (i) $f_{+}^{\eta}>0, f_{-}^{\eta}<0 ;(i i) 0<f_{+}^{\eta}<f_{-}^{\eta} ;($ iii $) f_{ \pm}^{\eta}<0 ;(i v) f_{ \pm}^{\eta} \in \mathbb{C},\left(f_{+}^{\eta}\right)^{*}=f_{-}^{\eta}$ that have to be studied separately.

In the case $(i)$ the quadratic form (3.9) is positive definite only when the first multiplier is positive: $f(\sigma)<f_{+}^{\eta}$ for all the $\sigma \in\left(\sigma_{I R}, \sigma_{U V}\right)$. Due to the fact that $|W(\sigma)|$ has in this 
interval one maximum $\sigma=\sigma_{I R}$, the above inequality is satisfied along the entire RG flow if:

$$
f\left(\sigma_{I R}\right)<f_{+}^{\eta} \quad \text { or equivalently } \quad L_{+}^{\eta}<L_{I R}
$$

thus introducing a certain minimal scale $L_{+}^{\eta}$ for this model ${ }^{29}$. We next consider the case (ii), where the condition that (3.9) is positive, requires that the two multipliers to have the same sign. When both are positives we find again the above minimal scale requirement, while in the case when both are negatives the restriction $f(\sigma)>f_{-}^{\eta}$ defines the new region of maximal scale $L_{-}^{\eta}$, i.e.

$$
f\left(\sigma_{U V}\right)>f_{-}^{\eta} \quad \text { or equivalently } \quad L_{-}^{\eta}>L_{U V},
$$

Finally, in the remaining two cases (iii) e (iv) the eq. (3.9) is automatically positive and no other restrictions on the values of $W(\sigma)$ are needed .

The next step of the proof consists in the ordering of all the $f_{\eta}^{ \pm}$'s within the intervals of admissible $\lambda$ values, where they are real and positive. The knowledge of their explicit forms (3.2) as certain functions of $\lambda$ and $d$ is indeed enough to solve analytically this problem. We find however its equivalent graphical solution to be much more efficient. The plots of all the $f_{\eta}^{ \pm}(\lambda)$ s for both $\mu_{ \pm}$models, for different values of $d$ and for specific intervals of values of $\lambda$ are given on Figs. (2(a))-(2(c)) and (3(a))-(3(b)). They provide the particular orderings of the $f_{\eta}$ 's for all the cases of interest, to be used in the proof of the $a / c$-Theorems in the Sects.3.3 and 3.4 below. One representative example is given by :

$$
f_{-}^{c^{\prime}}<f_{-}^{a}<0<f_{+}^{a}<f_{+}^{t o p}<f_{+}^{c^{\prime}}<f_{-}^{t o p}
$$

valid for $\mu_{+}$model in five dimensions and within the interval $\lambda \in(1 / 4,1 / 3)$ only, as one can see from fig.(2(c)). Once the ordering of all the positive $f_{\eta}^{ \pm}$'s is established, the last step in the proof consists in deriving the proper intersections between the restrictions we have found for the three distinct cases of $\eta=t o p, a, c^{\prime}$ 's.

The fact that two of the "scale ratios" $f_{ \pm}^{\eta}(\lambda, \mu)$ and also the corresponding "critical values" $\lambda_{\eta}^{(1,2)}$ explicitly depend on the space-time dimension $d$, makes also necessary to consider separately the proofs of the $a / c$-Theorems in the following three cases: (a) $d=5$; (b) The GB-limit $\mu=0$ and (c) $d \geq 7$. As we are going to show in the next subsections they give rise to rather different restrictions on the Lovelock couplings and on the values of the $W$ as well.

\section{3 $d=5$ Proof: Standard $a / c$-Theorem}

\subsection{1 $\mu_{+}$model}

The statement of the standard $a / c$-Theorem for the $\mu_{+}$model now reads:

\footnotetext{
${ }^{29}$ We recall that when $\sigma_{U V / I R}$ represent two consecutive extrema of $W(\sigma)$ such that $\left|W\left(\sigma_{U V}\right)\right|<|W(\sigma)|<$ $\left|W\left(\sigma_{I R}\right)\right|$, the restrictions on $W(\sigma)$ within the interval $\left(\sigma_{I R}, \sigma_{U V}\right)$ can be written as an equivalent requirement on the corresponding (boundary/horizon) values $\left|W\left(\sigma_{I R / U V}\right)\right|$.
} 
The $a(l)$ - and $c(l)$-functions are both positive and monotonically decreasing during the $R G$ flow between $\sigma_{U V}$ and $\sigma_{I R}$ only when the following restrictions:

$$
\begin{aligned}
& -\infty<\lambda<\frac{5}{27}: L_{I R}^{2}>\frac{L^{2}}{f_{+}^{t o p}\left(\lambda ; \mu_{+}\right)}, \quad f_{+}^{t o p}\left(\lambda ; \mu_{+}\right)=\frac{1}{3 \lambda}(1-\sqrt{1-3 \lambda}), \\
& -\frac{5}{27}<\lambda<\frac{1}{3}: L_{I R}^{2}>\frac{L^{2}}{f_{+}^{a}\left(\lambda ; \mu_{+}\right)}, \\
& f_{+}^{a}\left(\lambda ; \mu_{+}\right)=\frac{1}{3 \lambda+\sqrt{3 \lambda\left(1+3 \lambda+\frac{2}{3} \sqrt{1-3 \lambda}\right)+\frac{2}{3}(1-\sqrt{1-3 \lambda})}}
\end{aligned}
$$

are satisfied.

Proof. Following the $\mu_{+}$model description given in Sect.2.1., we first consider the (a1) case corresponding to $\lambda \in\left(-\infty, \frac{1}{4}\right)$ and $\mu_{+}(\lambda) \geq 0$.

(a) Reality conditions. It turns out that in this case $f_{ \pm}^{\text {top }}$ are always real: $f_{+}^{\text {top }}(\lambda)>0$, while $f_{-}^{t o p}(\lambda)<0$. We next realize that the condition $\lambda^{2} \geq \mu_{+}$, ensuring the reality of $f_{ \pm}^{a}\left(\lambda, \mu_{+}\right)$'s, can be rewritten as

$$
\left(\lambda-\frac{5}{27}\right)(\lambda+1) \geq 0
$$

Together with their explicit form (3.2), it leads to the conclusion that both $f_{ \pm}^{a}(\lambda)$ are real and negative for $\lambda<-1$ and real and positive for $\lambda \geq 5 / 27 .^{30}$ Finally, we find that the requirement $\lambda^{2} \geq 9 \mu$ for the reality of $f_{ \pm}^{c^{\prime}}\left(\lambda, \mu_{+}\right)$implies :

$$
\left(\lambda+9+\frac{16}{\sqrt{3}}\right)\left(\lambda+9-\frac{16}{\sqrt{3}}\right) \geq 0 .
$$

Then it becomes evident that both $f_{ \pm}^{c^{\prime}}<0$ are real and negative within the interval $\lambda \in\left(-\infty,-9-\frac{16}{\sqrt{3}}\right)$, while both are positive $0<f_{+}^{c^{\prime}}<f_{-}^{c^{\prime}}$ when $\lambda \in\left(-9+\frac{16}{\sqrt{3}}, 1 / 4\right)$.

(b) Ordering. According to our discussion in sect.3.2., the simplest method for establishing of the ordering of all the real positive $f_{ \pm}^{\eta}$ 's given by eqs. (3.2) and (3.10) is the graphical one. As one can see from their plots on the Figs. (2(a))-(2(b)), for the values of $\lambda$ within the interval $-\infty<\lambda<\frac{5}{27}$, only $f_{+}^{t o p}$ is positive, while for $\lambda \in\left(\frac{5}{27},-9+\frac{16}{\sqrt{3}}\right)$ we find that:

$$
f_{-}^{t o p}<0<f_{+}^{a}<f_{+}^{t o p}<f_{-}^{a}
$$

Finally as shown on Fig.(2(c)) for $\lambda \in\left(-9+\frac{16}{\sqrt{3}}, \frac{1}{4}\right)$ the following ordering :

$$
f_{-}^{t o p}<0<f_{+}^{a}<f_{+}^{t o p}<f_{+}^{c^{\prime}}<f_{-}^{c^{\prime}}<f_{-}^{a}
$$

takes place.

\footnotetext{
${ }^{30}$ Notice that at the "degenerate points" $\lambda_{ \pm c r}^{a}=-1,5 / 27$ the topological and a-scales do coincide, say $f_{+}^{a}(5 / 27)=f_{-}^{a}(5 / 27)=f_{+}^{t o p}(5 / 27)=9 / 5$, etc. and the corresponding dual CFT's have vanishing central charges $a\left(\lambda_{c r}\right)=0=c\left(\lambda_{c r}\right)$.
} 
(c) $f_{U V / I R}$ restrictions. Remember that the restrictions on the values of $f_{U V}$ and $f_{I R}$ (or $L_{U V / I R}$ ), for which the standard $a / c$-Theorem is satisfied are given by the common solutions of the following inequalities:

$$
\left(f-f_{-}^{t o p}\right)\left(f-f_{+}^{t o p}\right)<0, \quad\left(f-f_{-}^{a}\right)\left(f-f_{+}^{a}\right)>0, \quad\left(f-f_{-}^{c}\right)\left(f-f_{+}^{c}\right)>0,
$$

Taking into account eqs. (3.11) and (3.12), we conclude that:

- $-\infty<\lambda<\frac{5}{27}: \quad 0<f_{U V}<f_{I R} \leq f_{+}^{t o p}, \quad \bullet \frac{5}{27}<\lambda<\frac{1}{4}: \quad 0<f_{U V}<f_{I R} \leq f_{+}^{a}$.

represent all the solutions of the requirements (3.13).

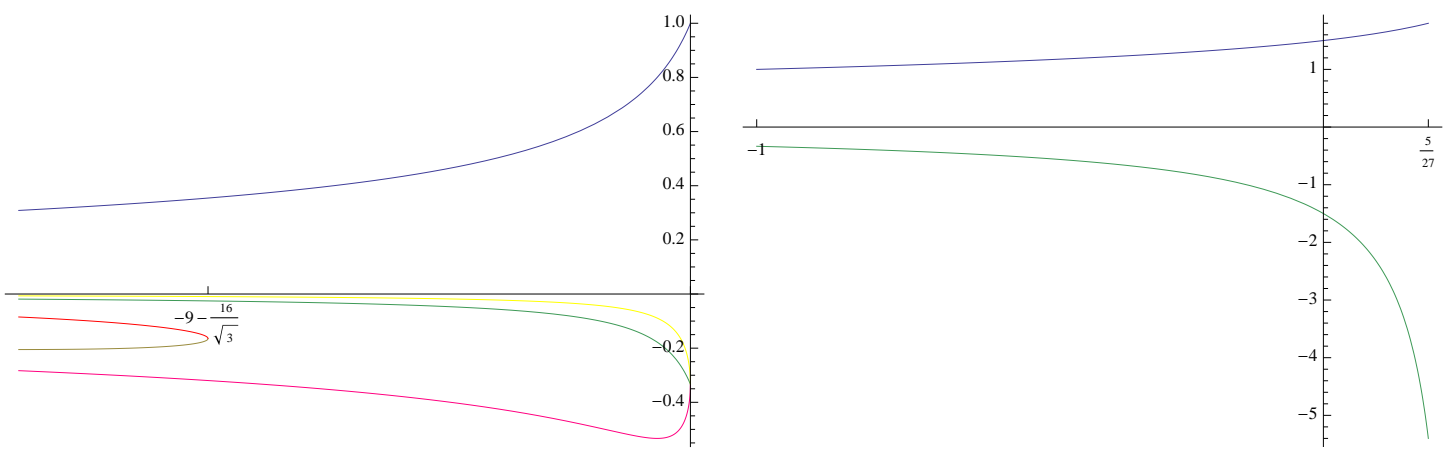

(a)

(b)

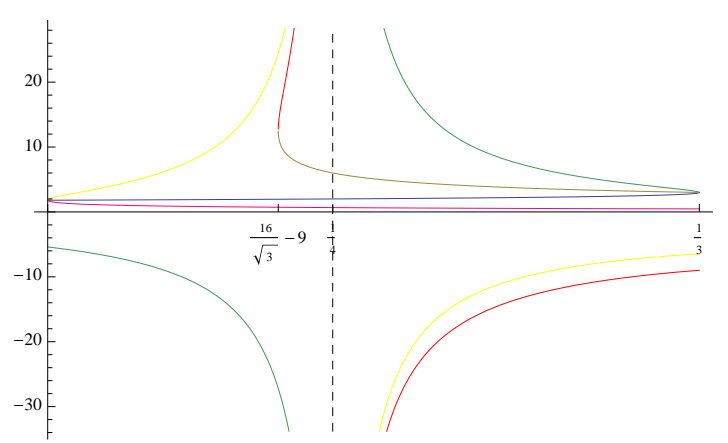

(c)

Figure 2. The $f_{ \pm}^{\eta}\left(\lambda ; \mu_{+}\right)$curves for $d=5: f_{+}^{t o p}$ is plotted in blue; $f_{+}^{a}$ in pink; $f_{+}^{c^{\prime}}$ is beige; $f_{-}^{t o p}$ in green; $f_{-}^{a}$ in yellow and $f_{-}^{c^{\prime}}$ in red. The $\lambda$ intervals are as follows: on Fig.(a) $\lambda<-1$ with $(-1,0)$ as reference point(r.p.); on Fig.(b) $-1<\lambda<5 / 27$ with $(0,0)$ as r.p.; and on Fig.(c) $5 / 27<\lambda<1 / 3$ with $(5 / 27,0)$ as r.p.

We next consider the $\mu_{+}$model within the interval $\frac{1}{4}<\lambda<\frac{1}{3}$, where $\mu_{+}<0$ and both $f_{ \pm}^{\text {top }}$ are now positive, i.e. the cases (a2) and (a3) of sect.2.1. Similar arguments as the ones given above, together with the plots of the curves $f_{ \pm}^{\eta}\left(\lambda, \mu_{+}\right)$presented on Fig.(2(c)) lead us to the conclusion that:

$$
f_{-}^{c^{\prime}}<f_{-}^{a}<0<f_{+}^{a}<f_{+}^{t o p}<f_{+}^{c^{\prime}}<f_{-}^{t o p}
$$

Due to the fact that now $\mu+$ is negative, the conditions for validity of the $a / c$-Theorem have the form (3.13), but now with inverted signs, i.e.:

$$
\left(f-f_{-}^{t o p}\right)\left(f-f_{+}^{t o p}\right)>0, \quad\left(f-f_{-}^{a}\right)\left(f-f_{+}^{a}\right)<0, \quad\left(f-f_{-}^{c}\right)\left(f-f_{+}^{c}\right)<0,
$$


Nevertheless, their solution is again given by

$$
\text { - } \frac{1}{4}<\lambda<\frac{1}{3}: \quad 0<f_{U V}<f_{I R} \leq f_{+}^{a},
$$

reflecting the corresponding new $f_{ \pm}^{\eta}\left(\lambda, \mu_{+}\right)$'s ordering (3.14).

Combining all these results we arrive at the statement of the standard $a / c$-Theorem announced at the beginning of this section.

\subsection{2 $\mu_{-}$model}

The $\mu_{-}$model corresponds to particular values (2.5) of $\mu_{-}(\lambda)$ for $\lambda \in(-\infty, 1 / 3)$.

Reality conditions. As shown in Sect.2.1., the $f_{ \pm}^{t o p}\left(\lambda ; \mu_{-}\right)$given by $(2.7)$ are both negative for all the negative values of $\lambda$. They become real and positive $0<f_{+}^{\text {top }}<f_{-}^{\text {top }}$ for $\lambda \in(0,1 / 3)$. Taking into account the explicit form (3.2) of $f_{ \pm}^{a}\left(\lambda, \mu_{-}\right)$and $f_{ \pm}^{c^{\prime}}\left(\lambda, \mu_{-}\right)$, we next realize that for $\lambda<1 / 3$ they are always real.

Ordering. As one can see from the Figs. (3(a)) and (3(b)), the following orderings of

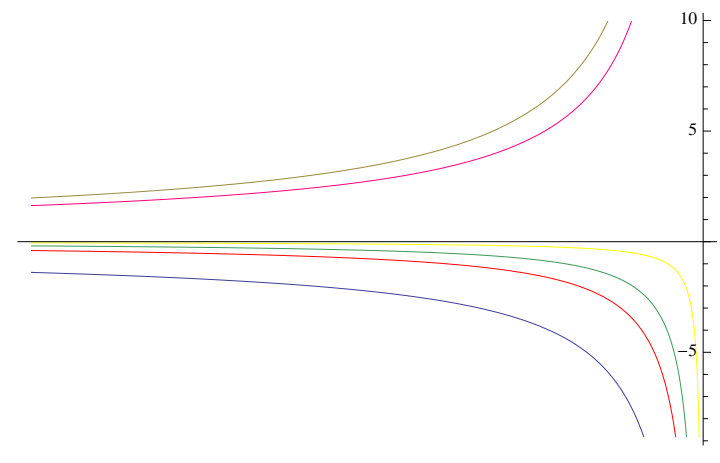

(a)

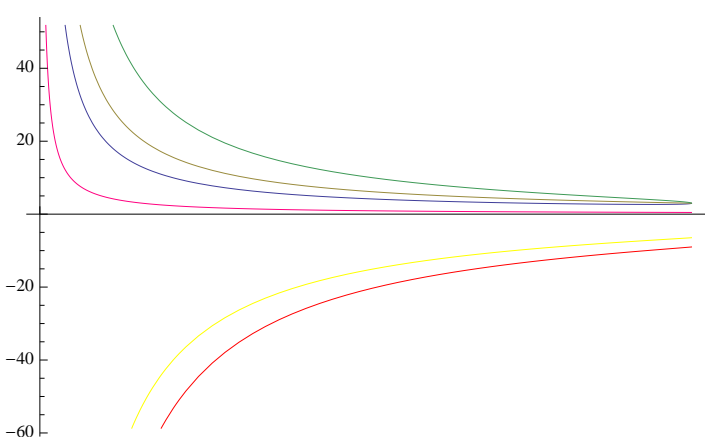

(b)

Figure 3. The $f_{ \pm}^{\eta}\left(\lambda ; \mu_{-}\right)$curves for $d=5: f_{+}^{\text {top }}$ is plotted in blue; $f_{+}^{a}$ in pink; $f_{+}^{c^{\prime}}$ in beige; $f_{-}^{\text {top }}$ in green; $f_{-}^{a}$ in yellow and $f_{-}^{c^{\prime}}$ in red. Fig.(a) corresponds to $\lambda<0$ and the Fig.(b) to $0<\lambda<1 / 3$.

$f_{ \pm}^{\eta}\left(\lambda, \mu_{-}\right)$'s take place: for $\lambda<0$ (see $\left.(3(\mathrm{a}))\right)$ we have

$$
f_{+}^{t o p}<f_{-}^{c^{\prime}}<f_{-}^{t o p}<f_{-}^{a}<0<f_{+}^{a}<f_{+}^{c^{\prime}},
$$

while for $0<\lambda<\frac{1}{3}$ (see $3(\mathrm{~b})$ ) we find that $f_{-}^{c}$ and $f_{-}^{a}$ are now negative and the remaining $f_{ \pm}^{\eta}$ 's are ordered as follows:

$$
f_{-}^{c^{\prime}}<f_{-}^{a}<0<f_{+}^{a}<f_{+}^{t o p}<f_{+}^{c^{\prime}}<f_{-}^{t o p}
$$

$f_{U V / I R}$ restrictions. Similarly to the $\mu_{+}$model in the region where $\mu_{+}<0$, the restrictions imposed on $f_{U V / I R}$ by the standard $a / c$-Theorem for the $\mu_{-}$model are again given by eqs. (3.15). They are satisfied for all the values of $f_{U V / I R}$ such that $0<f_{U V}<$ $f_{I R} \leq f_{+}^{a}$. Hence the $a / c$-Theorem statement for the five dimensional $\mu_{-}$model takes the following simple form: 
The condition

$$
\text { - }-\infty<\lambda<\frac{1}{3}: L_{I R}^{2}>\frac{L^{2}}{f_{+}^{a}\left(\lambda ; \mu_{-}\right)} .
$$

guarantees that both $a-$ and $c$-central charges are positive and monotonically decreasing during the $R G$ flow from $\sigma_{U V}$ to $\sigma_{I R}$.

\subsubsection{Topological versus $a$-scales}

The description of the models with arbitrary negative values of the Lovelock couplings $\lambda$ and $\mu$ (not necessarily related as in the case of $\mu_{-}$model) requires separate discussion. Due to the fact that now $f_{ \pm}^{\text {top }}$ are either both negative or complex, we realize that $c(f)>0$ for all $f$ 's without any restrictions. Hence in the absence of topological vacua no natural smallest or/and largest scales $L_{ \pm}^{t o p}$ exist. Indeed one can introduce as such a fundamental scale the smallest of the physical vacua scales by imposing $f_{I R}=1$. We have to remind however that the requirement $a(f)>0$ (in the case when one of the $f_{ \pm}^{a}$ is positive) introduces certain restrictions on the values of physical scales, namely $f_{U V / I R} \leq f_{-}^{a}$. As one can see from the explicit forms (3.2) of $f_{ \pm}^{a}$ and $f_{ \pm}^{c^{\prime}}$ for negative $\lambda$ and $\mu$ we have that: $0<f_{-}^{a}<f_{-}^{c^{\prime}}$. Then the Standard $a / c$-Theorem conditions lead to the following requirement:

$$
0<f_{U V}<f_{I R} \leq f_{-}^{a} \quad \text { or } \quad L_{U V}>L_{I R} \geq L_{-}^{a},
$$

with $f_{-}^{a}=L^{2} /\left(L_{-}^{a}\right)^{2}$ and $f_{I R}=L^{2} / L_{I R}^{2}$, thus introducing a minimal a-scale $L_{-}^{a}$. Therefore the most appropriate normalization in this case is to impose the condition $f_{-}^{a}=1$, which fixes the fundamental QT-Gravity scale as $L=L_{-}^{a}$. Similarly to the topological scale normalization, described in Sect.2.1., that leads to the specific relations (2.5) between the Lovelock couplings $\lambda$ and $\mu$, the choice $f_{-}^{a}=1$ give rise to the following linear relation:

$$
|\mu|=\frac{1}{3}+2|\lambda|, \quad \lambda<0, \quad \mu<0
$$

Then the $d=5 a / c$-Theorem requirements read: $0<f_{U V}<f_{I R}<1$. Notice that again we get the same UV/IR-physical scales restriction (3.19), which confirms the fact that the form of the restrictions on the physical scales does not depend on the choice of the fundamental QTG scale $L$.

An important comment concerning the nature of the $a$-scale is now in order. Notice that $L_{-}^{a}$ has rather different gravitational and $Q F T_{d-1}$ meaning compared to the one of the topological and physical scales. The $L_{ \pm}^{\text {top }}$ and $L_{U V / I R}$ scales are related to the cosmological constants $\Lambda_{U V / I R}=-(d-1)(d-2) / 2 L_{U V / I R}^{2}$ of the $A d S_{d}$ vacua solutions of QT Gravitymatter model (2.1). According to the AdS/CFT correspondence, these "vacua scales" define the central charges of the dual $C F T_{d-1}^{U V / I R}$ (see Sect.2.2. above) and geometrically they do correspond to the boundary/horizon's of the $(a) A d S_{d}$ DWs space-times. Instead, the $a$-scale $L_{-}^{a}$, which is characterized by the vanishing $a\left(f_{-}^{a}\right)=0$ central charge, in general is not related neither to the Lovelock gravity vacua $\sigma_{k}$ nor to the zeros of the $\beta$ - function of the dual $Q F T_{d-1}{ }^{31}$.

\footnotetext{
${ }^{31}$ excepts in the very special degenerate cases, when the $a$-scale coincides with one of the physical scales $L_{-}^{a}=L_{I R}$ or/and with one of the topological scales $L_{-}^{a}=L_{+}^{t o p}$. Such " $a$-critical points" $\sigma_{c r}^{a}$, i.e. when $a\left(\sigma_{c r}^{a}\right)=0$, do correspond to a QT Gravity vacuum dual to $C F T_{d-1}(\lambda=5 / 27)$ of vanishing central charges.
} 
Let us remind that the interpretation of $a(l)$ as an entanglement entropy in the dual $C F T_{d-1}[9,62,65]$ indicates that $a\left(\sigma_{c r}^{a}\right)=0$ is the end point of the consistent causal description in both the gravitational and its dual QFT models. In this context we should mention that our (smooth) DW's solutions for a large class of superpotentials $W(\sigma)$ in the cubic Quasi-Topological Gravity (2.1), do not provide arguments for an eventual interpretation of the zeros of $a(\sigma)$-central function as a new saddle point of the effective gravitational action, describing first order phase transitions in the dual $Q F T_{d-1}$, as suggested in ref. $[73]$ in the case of thin walls of GB gravity.

\subsection{Comments on $d=5 c$-Modified and $a$-Weaken Theorems}

The conditions of validity of the Standard $d=5 a / c$-Theorem established in Sect.3.3. above, provide certain $\lambda$ - and $\mu$-dependent restrictions on the values of the Superpotential and equivalently on the "physical scales" $L_{U V / I R}$, during the RG flow, such that both $a(l)$ and $c(l)$-central functions are positive and monotonically decreasing. The most important consequence of our analysis of the requirements (3.13) and (3.15) is that:

The $Q T G$-induced $d=5 c(l)$-central function is always decreasing, i.e. $\frac{d c}{d l}<0$ for all the allowed values of the Lovelock couplings and therefore no Modified Holographic cTheorem can exists in five dimensions.

This statement establishes a very strong non-perturbative restriction on the properties of the holographic c-function of the $a \neq c Q F T_{4}$ 's duals to the $d=5$ QT Gravity-matter model (2.1), thus excluding the possibility of existence of consistent dual $Q F T_{4}$ models of increasing or non-monotonic $c(l)$-functions ${ }^{32}$.

We next consider the $a$-Weaken version of the $d=5$ Theorem, when the restriction (3.10) for the $\mu_{+}$model, valid within the interval $\frac{5}{27}<\lambda<\frac{1}{3}$, is replaced by:

$$
0<f_{U V} \leq f_{+}^{a} \leq f_{I R} \leq f_{+}^{t o p},
$$

thus permitting to the $a(l)$-central function to change its sign at a specific " $a$-scale" $l_{c r}^{a}=L_{+}^{a}$. The corresponding "critical coupling" $\sigma_{c r}^{a} \in\left(\sigma_{I R}, \sigma_{U V}\right)$, determined by the real solutions of the equation $a\left(\sigma_{c r}^{a}\right)=0$, is dividing the former massless phase $\left(\sigma_{I R}, \sigma_{U V}\right)$ in two parts: $\left(\sigma_{I R}, \sigma_{c r}^{a}\right)$ of negative $a(l) \leq 0$ and the "physical" one $\left(\sigma_{c r}^{a}, \sigma_{U V}\right)$ with $a(l)>0$, where all the conditions of the Standard $a / c$-Theorem are satisfied. Indeed the consistent RG evolution of the $\mathrm{CFT}_{4}$ data terminates at $\sigma_{c r}^{a}$. Due to the fact that the former IR critical point $\sigma_{I R}$ is now placed in the "non-physical" region and also due to the presence of the new finite critical scale (and mass) $l_{c r}^{a}=L_{+}^{a}=1 / M_{c r}^{a}$, the $Q F T_{4}$-phase $\left(\sigma_{I R}, \sigma_{U V}\right)$ is not massless anymore (see Sect.7.4 below for more details and examples). The conclusion is that: when the parameters of the superpotential $W(\sigma)$ are satisfying the following weaker restrictions:

$$
\left(L_{t o p}^{2}\right)_{+}<L_{I R}^{2} \leq\left(L_{a}^{2}\right)_{+}<L_{U V}^{2},
$$

then the $a$-Weaken form of the Theorem holds for the $\mu_{+}$-model within the interval $\lambda \in$ $(5 / 27,1 / 3)$. Note that we are excluding the case $L_{t o p}^{2}<L_{I R}^{2}<L_{U V}^{2} \leq L_{a}^{2}$ at all, i.e. when

\footnotetext{
${ }^{32}$ notice that according to refs. [42, 43, 47] , based on perturbative 1-loop calculations in a class of $\mathcal{N}=1,2$ supersymmetric $Q F T_{4}$, such behaviour of $c(l)$ is not in principle forbidden.
} 
the $a(l)$ is negative within the entire interval $\left(\sigma_{I R}, \sigma_{U V}\right)$, since no consistent RG flows (and dual $Q F T_{4}$ 's) can be defined for those values of the coupling.

Let us also mention the remarkable fact that the $Q F T_{4}$ 's duals to the $\mu_{+}$-model considered within the interval $\lambda \in(-\infty, 5 / 27)$ have to satisfy the Standard Holographic a/cTheorem. Due to the absence of critical $a$-scales for these values of the Lovelock couplings, the $a(l)$-central function is always positive and monotonically decreasing and the only permitted Holographic RG flows $U V \rightarrow I R$ are the massless ones. Therefore in the case of the $\mu_{+}$-model there exists one special point $\lambda_{c r,+}^{a}=5 / 27,{ }^{33}$ which splits the interval of the allowed values of $\lambda$ in two subintervals $(-\infty, 5 / 27)$ and $(5 / 27,1 / 3)$. For the values of $\lambda$ belonging to each one of these intervals one can realize qualitatively different Holographic RG flows and the corresponding dual $Q F T_{4}$ 's have rather different phase structure as it is shown in Sect.7.4. below. Similar statement is valid for the $\mu_{-}$model with $\lambda_{c r}^{a}=0$.

\section{Gauss-Bonnet $a / c$-Theorems}

The Gauss-Bonnet Gravity (i.e. the $\mu=0$ QTG model) coupled to scalar matter for $d \geq 5$ is known to represent the simplest consistent gravity model, which in the context of the $A d S / C F T$ correspondence give rise to specific $a \neq c C F T_{d-1}$ 's. The requirements of the corresponding GB " $a$-Theorem", together with the $c(l)>0$ positivity restrictions have been established in refs. $[8,9,26]$. The recent construction of the GB flat domain walls [5] provides an efficient tool for the description of the Holographic RG flows in the corresponding dual $Q F T_{d-1}$ (see sect.7.3. below). In this section we shall derive the conditions we have to impose on the matter superpotential $W$, necessary for the validity of the $d \geq 5$ GB Holographic $a / c$-Theorems.

As is well known, the monotonic decreasing of the $a(l)$ - central function is automatically satisfied once the condition $W<0$ is imposed. The starting point of our analysis of the $a(l)$ and $c(l)$ positivity conditions, as well as of the monotonicity of $c(l)$, are the following explicit forms of these quantities:

$$
\begin{aligned}
& c(\sigma)=\left(\frac{L}{(d-2) l_{p l} \sqrt{f}}\right)^{d-2}(1-2 \lambda f), \quad a(\sigma)=\left(\frac{L}{(d-2) l_{p l} \sqrt{f}}\right)^{d-2}\left(1-2 \lambda\left(\frac{d-2}{d-4}\right) f\right), \\
& \frac{d c}{d l}=-\frac{2(d-2)^{d}}{\kappa^{2} l_{p l}^{d-2}}\left(\frac{W^{\prime}(\sigma)}{W(\sigma)}\right)^{2} c(l)\left(1-2 \lambda\left(\frac{d-4}{d-2}\right) f\right)
\end{aligned}
$$

We next introduce the GB counterparts $f_{G B}^{\eta}=L^{2} /\left(L_{G B}^{\eta}\right)^{2}$ of the "critical" values of $f(\sigma)$ that are determined by the corresponding zeros of $c\left(f_{G B}^{t o p}\right)=0=a\left(f_{G B}^{a}\right)$ and $\frac{d c}{d l}\left(f_{G B}^{c^{\prime}}\right)=0$ :

$$
f_{G B}^{t o p}=\frac{1}{2 \lambda}, \quad f_{G B}^{a}=\frac{1}{2 \lambda}\left(\frac{d-4}{d-2}\right), \quad f_{G B}^{c^{\prime}}=\frac{1}{2 \lambda}\left(\frac{d-2}{d-4}\right)
$$

Notice that for $\lambda>0$ and $d \geq 5$ the $f_{G B}^{\eta}$ 's are ordered as follows:

$$
0<f_{G B}^{a} \leq f_{G B}^{t o p} \leq f_{G B}^{c^{\prime}}
$$

\footnotetext{
33 where the topological and $a$-scales coincide $: f_{+}^{a}(5 / 27)=f_{-}^{a}(5 / 27)=f_{+}^{t o p}(5 / 27)=9 / 5$. This model describes a particular $\mathrm{CFT}_{4}$ with vanishing central charges $a\left(\lambda_{c r}^{a}\right)=0=c\left(\lambda_{c r}^{a}\right)$.
} 
Then the conditions of validity of the standard $d \geq 5 \mathrm{~GB} a / c$-Theorem:

$$
0<f \leq f_{G B}^{a}, \quad 0<f \leq f_{G B}^{t o p}, \quad 0<f \leq f_{G B}^{c^{\prime}}
$$

take the following simple form:

$$
0<f_{U V}<f_{I R} \leq f_{G B}^{a}=\frac{1}{2 \lambda}\left(\frac{d-4}{d-2}\right), \quad L_{U V}>L_{I R} \geq L_{G B}^{a} \geq L_{G B}^{t o p}
$$

For negative values of $\lambda<0$, all the conditions (4.4) for validity of the standard $a / c$ Theorem: $a>0, c>0$ and $d a / d l<0, d c / d l<0$ are automatically satisfied without any restrictions on the physical scales $L_{U V / I R}$. In the absence of topological or $a$-scales, the natural choice of the normalization is the smallest physical vacua scale $L=L_{I R}$.

- Comments on the normalization. It is worthwhile to remind the restrictions on the GB coupling $\lambda$ related to the reality and positivity conditions imposed on the solutions $f_{ \pm}$of the $G B$ vacua equation: $h=f(1-\lambda f)$ with $h>0$. In the case we are considering $\lambda>0$, the additional requirement is given by: $\lambda \leq 1 / 4 h$. We next observe that the $h\left(f_{G B}^{\text {top }}\right)=1 / 4 \lambda=L^{2} / L_{0 t o p}^{2}$ defines the maximal value of $h(f)$. Therefore we can relate the "fundamental scale" $L(2.1)$ to the minimal scale $L_{I R}$ introduced by the matter interaction, i.e. we can take $h_{I R}=h\left(f_{I R}\right)=1$ as "normalization":

$$
h\left(f_{G B}^{t o p}\right)>h_{I R}=1>h_{U V} \quad \text { or equivalently } \quad \lambda<1 / 4
$$

Notice that the above "physical vacua normalization" together with the $a / c$-Theorem condition (4.5) leads to the following stronger restriction on the values of $\lambda$ :

$$
0<\lambda \leq \frac{d(d-4)}{4(d-2)^{2}}<\frac{1}{4}
$$

where we have used the explicit form of the solution for the IR vacua equation:

$$
f_{I R}=\frac{1-\sqrt{1-4 \lambda h_{I R}}}{2 \lambda}
$$

One can use an alternative normalization $h\left(f_{\text {top }}\right)=1$ (see [5]), based on the topological scale $L=L_{G B}^{0 t o p}$, that fixes $\lambda=1 / 4$. In this case we get as a consequence of the requirement (4.5) instead of eq. (4.6) a stronger restriction on the allowed values of $h_{I R}=L_{0 t o p}^{2} / L_{0 I R}^{2}$ :

$$
0<h_{U V}<h_{I R} \leq \frac{d(d-4)}{(d-2)^{2}}<h_{t o p}=1,
$$

In fact for $\lambda>0$ both normalizations: $h_{\text {top }}=1$ and $h\left(f_{I R}\right)=1$ are equivalent and they reproduces the same restrictions on the physical scales $L_{U V / I R}$.

For negative $\lambda$ we have not topological scales at all (since $c>0$ for all the $\lambda<0$ ) and the only available normalization is the one related to the IR vacua scale, i.e. $h_{I R}=1$.

- $a$-Weaken GB Theorem. Similarly to the case of QT Gravity Holographic a/cTheorems, one can consider the $a$-Weaken form of the Theorem for positive values of $\lambda<1 / 4$ :

$$
\left(L_{t o p}^{2}\right)_{G B}<L_{I R}^{2} \leq\left(\frac{d-2}{d-4}\right)\left(L_{t o p}^{2}\right)_{G B}<L_{U V}^{2}
$$


thus allowing the $a(l)$-central function to change its sign at some $\sigma=\sigma_{c r}^{a} \in\left(\sigma_{I R}, \sigma_{U V}\right)$. For these $a$-Weaken conditions, the corresponding dual $Q F T_{d}$ has a consistent description only for the couplings within the interval $\sigma \in\left(\sigma_{c r}^{a}, \sigma_{U V}\right)$, which is not any more describing a massless phase, due to the existence of the $a$-mass scale $M_{G B}^{a}=1 / L_{G B}^{a}$.

We conclude the discussion of the GB Holographic $a / c$-Theorems by mentioning the important fact that for all the allowed values of GB coupling $\lambda \in(-\infty, 1 / 4)$, for arbitrary dimensions $d \geq 5$ and for rather general forms of the matter superpotential $W(\sigma)<0$ no modified $c$-Theorem can take place, i.e. the only possibility we can consistently realize is the following: $a>0, c>0$ and the $a$ - and $c$-central functions are both monotonically decreasing.

\section{Holographic $d \geq 7 a / c$ - Theorems}

\section{$5.1 \mu_{ \pm}$models Standard Theorem}

The proof of the $d \geq 7 a / c$-Theorem follows the same logics and we use the same methods of the $d=5$ case. Let us begin with the statement of the Standard $a / c$-Theorem valid for $d \geq 7$. According to our analysis of the conditions for validity of the Theorem, presented in App. A.1, for the $\mu_{+}$model we get the following requirements:

The conditions that $a(l)$ - and $c(l)$-central functions of the dual $Q F T_{d-1}$ 's are both positive and monotonically decreasing during the $R G$ flow between two consecutive critical points $\sigma_{U V}$ and $\sigma_{I R}$ are given by:

$$
\begin{array}{ll}
(1) \bullet & -\infty<\lambda<\frac{1}{4}: \quad f_{I R}<f_{+}^{a}\left(\lambda ; \mu_{+}\right), \\
(2) \bullet & \frac{1}{4}<\lambda<\frac{d(d-4)}{3(d-2)^{2}}: \quad f_{I R}<f_{+}^{a}\left(\lambda ; \mu_{+}\right) \text {or } f_{U V}>f_{-}^{c^{\prime}}\left(\lambda ; \mu_{+}\right), \\
(3) \bullet & \frac{d(d-4)}{3(d-2)^{2}}<\lambda<\frac{1}{3}: \quad f_{I R}<f_{+}^{t o p}\left(\lambda ; \mu_{+}\right) \text {or } f_{U V}>f_{-}^{c^{\prime}}\left(\lambda ; \mu_{+}\right) .
\end{array}
$$

The main difference in the Proof of this Theorem, when compared to the $d=5$ case, comes from the new intervals of $\lambda$ where $f_{ \pm}^{\eta}$ are real positive or complex. They are a consequence of the different sign of the factor $\left(\frac{d-4}{d-6}\right)$ multiplying $\mu$ in both $\frac{d c(\sigma)}{d l}$ and $a(\sigma)$-functions. This fact also causes modifications in the form (3.13) and (3.15) of $f^{\eta}$ 's restrictions (3.13) and (3.15) for $\eta=a, c^{\prime}$, while keeping unchanged the $c>0$ one. Another important change concerns the new orderings of the $f_{ \pm}^{\eta}$ 's. Let us also mention the presence of the new intermediate scale ${ }^{34} L_{-}^{h}$, introduced by $f^{h}=L^{2} /\left(L_{-}^{h}\right)^{2}$ in the regions of negative $h<0$ that leads to certain differences between the cases $d=7, d=8$ and $d \geq 9$ as described by eqs. (A.10) and (A.11) of App. A. It turns out that the requirements of the corresponding standard $a / c$-Theorem remain in fact the same for all $d \geq 7$. However when the modified $c$ Theorem is considered - thus permitting, say of increasing $c(l)$, which imposes $f_{+}^{c^{\prime}}<f<f_{-}^{c^{\prime}}$ - one can find different restrictions for $f_{U V / I R}$ in the maximal scale region depending on

\footnotetext{
${ }^{34}$ defined by $f^{h}=-\lambda / 2 \mu_{+}+\sqrt{\left(\lambda^{2}+4 \mu_{+}\right) / 4 \mu_{+}^{2}}$ which represents the greatest root of corresponding $h(f)=0$ equation
} 
the space-time dimensions and on the different intervals of values of $\lambda$, as for example in the $d=7$ case:

$$
\text { - } \lambda \in(7 / 25,8 / 27): \quad f^{h}<f<f_{-}^{c^{\prime}}, \quad \bullet \lambda \in(8 / 27,1 / 3): \quad f_{-}^{t o p}<f<f_{-}^{c^{\prime}}
$$

where we have now both the minimal and maximal scale restrictions together. The discussion of the conditions of validity of the holographic modified $c$-Theorem is presented in Sect.5.2. below.

Similar considerations lead to the following set of restrictions on the allowed values of $f_{U V / I R}$ in the case of the $\mu_{-}$model:

(1)In the region of negative $\lambda<0$ and $\mu<0$, according to the definitions (3.2) all the $f^{\eta}$ 's for $d \geq 7$ are either negative or complex. Therefore we have no any restrictions for the validity of the Standard Theorem, and no natural fundamental scale exists, differently from the $d=5$ case, where we have $f_{-}^{a}>0$ that introduces $L_{-}^{a}$ as a minimal fundamental scale.

(2)In the interval $\lambda \in(0,1 / 3)$, the conditions under which the Standard $a / c$-Theorem is satisfied are given by:

- $d=7,8: \quad 0<\lambda<\frac{1}{3}: \quad f<f_{+}^{\text {top }}\left(\lambda ; \mu_{-}\right) \quad$ or $\quad f>f_{-}^{c^{\prime}}\left(\lambda ; \mu_{-}\right)$,

- $d \geq 9: \quad 0<\lambda<\frac{(d-8)(d-4)}{3(d-6)^{2}}: \quad f<f_{+}^{a}\left(\lambda ; \mu_{-}\right) \quad$ or $\quad f>f_{-}^{c^{\prime}}\left(\lambda, \mu_{-}\right)$

- $d \geq 9: \frac{(d-8)(d-4)}{3(d-6)^{2}}<\lambda<1 / 3: \quad f<f_{+}^{t o p}\left(\lambda ; \mu_{-}\right) \quad$ or $\quad f>f_{-}^{c^{\prime}}\left(\lambda ; \mu_{-}\right)$

where $f$ is denoting $f_{I R}$ or $f_{U V}$ in the case of minimal/maximal scale correspondingly. The detailed derivation of the above requirements is presented in App. A.2.

\subsection{Maximal scale and Modified $c$-Theorem}

The analysis of the conditions under which the Standard $d \geq 7$ Holographic $a / c$-Theorem holds, reveals one interesting new feature, namely the existence of distinct domains of values of the Lovelock couplings $\lambda$ and $\mu_{ \pm}(\lambda)$, where the allowed physical scales $f_{U V / I R}$ are determined by certain maximal scale restrictions. Thus the corresponding dual $Q F T_{d}$ 's with $a \neq c$ can have arbitrarily large values of both central functions. These new properties reflect the possibility that for $d \geq 7$ we can have both $f_{ \pm}^{t o p}(\lambda)$ 's and $f_{ \pm}^{c^{\prime}}(\lambda)$ 's positive. Therefore we can appropriately choose the parameters of the matter superpotential $W(\sigma)$ such that the $a / c$-Theorems can be satisfied in two well separated regions of minimal or maximal scales restrictions as it shown in Sects.5.1.and App. A. The common feature of all these $d \geq 7$ models is that the minimal scale restrictions are always present and they are almost identical to those of the $d=5$ models and of the $d \geq 7$ GB models.

Another important fact to be mentioned is that the proofs of the Standard $d \geq 7$ Theorems provide all the ingredients needed in the descriptions of the specific restrictions that give rise to the corresponding $a$-Weaken and Modified c-Theorems.

$a$-Weaken Theorem. According to the analysis of Sect.3 and App. A, the conditions of the $a$-Weaken Theorem can be realized always when we have at least one $a$-scale $(i . e$. one 
positive $f_{ \pm}^{a}$ is present) and in the region of the minimal scale only. Notice that when both $f_{ \pm}^{a}>0$ are positive, the following ordering : $0<f_{+}^{a}<f_{+}^{t o p}<f_{-}^{a}<f_{-}^{\text {top }}$ takes place. This leads to the important conclusion that: it is impossible to realize the a-Weaken theorem in the region of the maximal scale, i.e. for $f>f_{-}^{t o p}$. We next separate the cases of one or two a-scales, where the conditions of the $a$-Weaken Theorem :

$$
0<f_{U V} \leq f_{+}^{a} \leq f_{I R} \leq f_{+}^{t o p}
$$

can be satisfied. Taking into account the results presented in App. A, we deduce the following list of models

- $\mu_{-} \operatorname{model}(d \geq 9): 0<\lambda \leq \frac{(d-8)(d-4)}{3(d-6)^{2}}, \quad \bullet \mu_{+} \operatorname{model}(d \geq 7):-\infty<\lambda \leq \frac{d(d-4)}{3(d-2)^{2}}$

that admit the $a$-Weaken version of the Theorem. Notice that for the $\mu_{-- \text {-model and for }}$ $d=7,8$ no $a$-Weaken Theorem holds.

Modified c-Theorem. As we have already mentioned, the modifications permitting monotonically increasing and non-monotonic $c(l)$-central functions are allowed only in the case when the maximal scale $L_{-}^{c^{\prime}}$ exists, i.e. for $f_{-}^{t o p / h}<f_{U V / I R}<f_{-}^{c^{\prime}}$. Then the standard $a / c$-Theorem requirements $f_{-}^{c^{\prime}} \leq f_{U V}<f_{I R}$ can be replaced by one of the following new conditions:

- increasing $c(l): f_{-}^{t o p / h} \leq f_{U V}<f_{I R} \leq f_{-}^{c^{\prime}}$

- non-monotonic $c(l): f_{-}^{t o p / h} \leq f_{U V} \leq f_{-}^{c^{\prime}}<f_{I R}$

that give rise to the $c$-Modified version of the Theorem. According to the results of App. A., one can realize these modifications in the case of $\mu_{-}$-model for all the values of $\lambda \in(0,1 / 3)$ and for all $d \geq 7$. Instead for the $\mu_{+}$model one can have $Q F T_{d-1}$ models satisfying c-Modified Holographic Theorem for all the $d \geq 7$ only within the intervals:

$$
\begin{array}{ll}
\bullet \frac{1}{4}<\lambda<\frac{8}{27}: & f^{h} \leq f_{U V}<f_{I R} \leq f_{-}^{c^{\prime}} \quad \text { or } \quad f^{h} \leq f_{U V}<f_{-}^{c^{\prime}} \leq f_{I R} \\
\bullet \frac{8}{27}<\lambda<\frac{1}{3}: & f_{-}^{t o p} \leq f_{U V}<f_{I R} \leq f_{-}^{c^{\prime}} \quad \text { or } \quad f_{-}^{t o p} \leq f_{U V}<f_{-}^{c^{\prime}} \leq f_{I R}
\end{array}
$$

Observe that for $\lambda$ within the interval $(-\infty, 1 / 4)$, we have no maximal scale at all and therefore no Modified c-Theorems can be realised for the $\mu_{+}$-model. Note also that in all the cases of monotonically increasing $c(l)$-central function, the $f_{U V / I R}$ 's have to respect the two scales restriction, i.e. their values are restricted between the minimal $L_{-}^{c^{\prime}}$ and the maximal scales, say $L_{-}^{t o p}$ or $L_{-}^{h},{ }^{35}$. Instead in the case of non-monotonic $c(l)$ we have only one relevant scale - the maximal $L_{-}^{\text {top/h}}$ one.

Our final comment is about the differences between the three distinct conditions on the $c(l)$ monotonicity properties, in the case when the maximal scale does exist. Due to the lack of clear physical interpretations of the $L_{-}^{c^{\prime}}$ as a fundamental maximal scale, it is

\footnotetext{
${ }^{35}$ remember that the definition of the new maximal "h-scale" in the case of negative $h<0$ is given by $f^{h}=L^{2} /\left(L_{-}^{h}\right)^{2}$
} 
evident that the most consistent physical description of the dual $Q F T_{d-1}$ is represented by the case of non-monotonic $c(l)$, where the $a / c$-Theorem restrictions can be formulated in terms of the topological scale $L_{-}^{\text {top }}$ only. The description of the dual QFT's that in the region of maximal scale are satisfying the conditions of the Standard (i.e. $d c / d l<0$ ) or of the Modified $d c / d l>0$ Holographic Theorems is involving additional scales that have not well established Quasi-Topological Gravity meaning. Instead in the case when $W$ is satisfying the minimal scale restrictions, the Standard $a / c$-Theorem is the only one that can be formulated in the terms of the well defined physical scales.

It is worthwhile to also mention the fact that the case $d=5$ is the only space-time dimension, for which no maximal scale exists and we can realize the restrictions imposed by the Standard $a / c$-Theorem only.

\section{Energy Fluxes Positivity and $a / c$-Theorems}

The RG evolution of the central charges $a$ and $c$ and of the conformal dimensions $\Delta_{\Phi}$, established by the Holographic $a / c$-Theorems in Sects.3-5, represents an important ingredient in the description of the massless phases in the $Q F T_{d-1}$ 's duals to Quasi-Topological Gravity (2.1). Based on the explicit form of the holographic $\beta_{W}$-function (2.8), they provide the conditions on the physical scales $L_{U V / I R}$ and on the gravitational couplings $\lambda$ and $\mu$ (i.e. on the $\left.L_{ \pm}^{\text {top }}\right)$ in order to have certain desired relations between $C F T_{U V}$ and $C F T_{I R}$ data and preserving the unitarity consistency of the both CFT's. This section is devoted to the analysis of the additional restrictions on the values of $L_{U V / I R}, \lambda$ and $\mu$ imposed by the requirements of unitarity and causality of the $C F T_{U V / I R}$ 's, coming from the remaining part of the CFT's data and to the RG features of this new conformal data. Namely, the problem concerns the investigation (1) of the properties of the structure constants of the $T_{i j}\left(x_{n}\right) T_{k l}(0)$ OPE's, i.e. the parameters $\mathcal{A}, \mathcal{B}$ and $\mathcal{C}$ of the stress-energy 3-point functions and (2) of the new restrictions on the $f_{U V / I R}$ related to the energy fluxes positivity (p.e.f.) conditions that $\mathcal{A}_{\mathcal{U V} / \mathcal{I} \mathcal{R}}, \mathcal{B}_{\mathcal{U V} / \mathcal{I R}}$ and $\mathcal{C}_{\mathcal{U V} / \mathcal{I R}}$ have to satisfy $[7,8,14-16,66]$.

\subsection{More on $C F T_{d-1}$ data: positive energy fluxes conditions}

The energy flux (per unit solid angle $\Omega_{d-3}$ ) measured in the future null infinity in the direction $n^{i}$ is given by the following "energy one-point functions":

$$
<\mathcal{E}(\vec{n})>_{\mathcal{O}}=\frac{<0\left|\mathcal{O}^{\dagger} \mathcal{E}(\vec{n}) \mathcal{O}\right| 0>}{<0\left|\mathcal{O}^{\dagger} \mathcal{O}\right| 0>}
$$

As explained in refs. $[8,14,16]^{36}$, in each unitary $C F T_{d-1}$ and for the states $\left|p>\sim \mathcal{O}_{p}\right| 0>$ created by the spacial components of $T_{i j}$ of helicities $\mathrm{p}=2,1,0$ (i.e. tensor, vector and scalar w.r.t. $S O(d-3)$ subgroup), the corresponding energy fluxes can be calculated by taking appropriate limits of the integrated 3- and 2-point functions of the stress tensor. The final

\footnotetext{
${ }^{36}$ we are closely following the results and the definitions of [8], especially those of Sect.3 and App. A, but with slightly different normalization of $c$ and $a$ and with $d$ replaced by $d-1$.
} 
result (see eqs. (3.6) and (3.32-34) of ref. [8]) can be written in the following simple form:

$$
\begin{aligned}
& E_{s}=<\mathcal{E}(\vec{n})>_{\text {scalar }}=\frac{E}{\Omega_{d-3}}\left(1+\frac{d-4}{d-2} t_{2}+\frac{d(d-3)-2}{d(d-2)} t_{4}\right), \\
& E_{v}=<\mathcal{E}(\vec{n})>_{\text {vector }}=\frac{E}{\Omega_{d-3}}\left(1-\frac{d-4}{2(d-2)} t_{2}-\frac{2}{d(d-2)} t_{4}\right), \\
& E_{t}=<\mathcal{E}(\vec{n})>_{\text {tensor }}=\frac{E}{\Omega_{d-3}}\left(1-\frac{1}{d-2} t_{2}-\frac{2}{d(d-2)} t_{4}\right),
\end{aligned}
$$

where $E$ denotes the total energy and the new coefficients $t_{2}$ and $t_{4}$ are parametrized by $\mathcal{A}$, $\mathcal{B}$ and $\mathcal{C}^{37}$. When $c \neq 0$, one can choose equally well $a, c$ and $t_{4}$ as independent parameters.

It is then evident that the natural requirement of positivity of the energy fluxes related to the above considered "tensor, vector and scalar states"

$$
E_{s} \geq 0, \quad E_{v} \geq 0, \quad E_{t} \geq 0
$$

lead to certain important restrictions on the values of $t_{2}$ and $t_{4}$, which together with the $c \geq 0$ condition provide strong requirements on the physically allowed values of $\mathcal{A}, \mathcal{B}$ and $\mathcal{C}$. For example, for all the GB induced CFT's and, more generally, for all the $\mathcal{N}=1$ super-symmetric $C F T_{d-1}$ the parameter $t_{4}^{G B}=0$ is always vanishing $[14,15]$ and the corresponding p.e.f. conditions take the form:

$$
-\frac{d-2}{d-4} \leq t_{2} \leq \frac{d-1}{2}
$$

The Holographic gravitational d-dimensional counterparts of the above field-theoretical $C F T_{d-1}$ p.e.f. requirements (6.2) are known to coincide with the causality conditions needed to avoid the propagation of superluminal signals (out of the light-cone of the $A d S_{d}$ boundary) for both the black-holes and shock wave backgrounds in the dual GB or QT Gravity models $[7,8,15,16]$. The problem of the causal consistency of the considered DW's backgrounds as well as of their stability require further investigations of the properties of the linear fluctuations of the metrics and of the matter field $\sigma$ that are out of the scope of the present paper.

Let us also mention that the "new $C F T$ data" (as well as the "old" one): $\mathcal{A}, \mathcal{B}, \mathcal{C}, a$ and $c$ turns out to have an equivalent "holographic" realization in terms of the fundamental scale $L$ and of the $f_{U V / I R}=L^{2} / L_{U V / I R}^{2}$ only. Their explicit form indeed depends on the details of the given GB and QTG models $[9,10]$. Therefore the p.e.f. conditions lead to certain restrictions on the critical values of $f$ or $W(\sigma)$ and on the $\lambda$ and $\mu$ values as well. They are introducing new upper bounds $f_{\max }=f_{ \pm}^{e p}$,

$$
0<f_{U V}<f_{I R}<f_{\max }
$$

to be compared with the minimal scale $f_{ \pm}^{a}$ and $f_{ \pm}^{\text {top }}$-bounds (3.2), derived in Sect.3. Taking into account the well known $A d S / C F T$ identification $\left(\frac{L_{k}}{l_{p l}}\right)^{d-2} \approx N_{c}^{2}$ of the vacua scales $L_{U V / I R}$ with the color number $N_{c}^{U V / I R}$ of the corresponding $S U\left(N_{c}\right)$ CFT's we can find a lower bound for $N_{c}^{I R}$ at the IR-end of the RG flow.

\footnotetext{
${ }^{37}$ the corresponding explicit formulas $t_{K}(\mathcal{A}, \mathcal{B}, \mathcal{C}), K=2,4$ are given by eq. (3.9) of ref. [8] with $d \rightarrow d-1$ and they lead to the equivalent form (A.17-18) of $<\mathcal{E}(\vec{n})>_{p}$ in terms of $\mathcal{A}, \mathcal{B}$ and $\mathcal{C}$ only.
} 


\subsection{Holographic GB $t_{2}$-Theorem}

6.2.1.New minimal scales for the a/c-Theorem. The $C F T_{d-1}$ 's duals to GB Gravity are characterized by $t_{4}=0$ and as a consequence all the remaining data: $t_{2}$ and $\mathcal{A}, \mathcal{B}, \mathcal{C}$, can be parametrized by the central charges $a$ and $c$ only:

$$
t_{2}=\frac{(d-1)(d-2)}{(d-3)}\left(1-\frac{a}{c}\right)
$$

Then the p.e.f. requirements (6.4) take the following form

$$
\frac{(d-1)(d-4)}{d(d-4)+1} \leq \frac{c}{a} \leq \frac{d-1}{2}
$$

thus restricting the ratio of the central charges $c / a$ to belong to the well known $\mathcal{N}=1$ SUSY unitary $C F T_{d-1}$ 's window [8-10, 14]. Taking into account the explicit form (4.1) of the GB central charges in terms of the $f_{U V / I R}$, we next derive the new upper bounds $f_{ \pm}^{e p}$ imposed by the p.e.f. conditions:

$$
\begin{array}{ll}
-\lambda>0: & 0<f_{U V}<f_{I R}<\frac{(d-3)(d-4)}{2 \lambda\left(d^{2}-5 d+10\right)}=f_{+}^{e p}<f_{G B}^{a}, \quad L_{U V}^{2}>L_{I R}^{2}>L_{e p}^{2}=\frac{L^{2}}{f_{+}^{e p}}, \\
\text { - } \lambda<0: & 0<f_{U V}<f_{I R}<\frac{(d-3)}{2|\lambda|(d+1)}=f_{-}^{e p}, \quad L_{U V}^{2}>L_{I R}^{2}>L_{e p}^{2}=\frac{L^{2}}{f_{-}^{e p}},
\end{array}
$$

They introduce specific new constraints on the allowed physical scales $L_{U V / I R}$, characterizing the UV-and IR- $C F T_{d-1}$ 's that represents the limiting critical points $\sigma_{U V / I R}$ of the massless RG flow in consideration. Similarly to the restrictions (4.6) on the values of $\lambda_{G B}$, introduced by the $a$-scale $f_{-}^{a}$ (see sect.4), the above p.e.f. conditions (6.7) and (6.6) can be rewritten in an equivalent form :

$$
-\frac{(d-3)(3 d-1)}{4(d+1)^{2}} \leq \lambda h_{I R} \leq \frac{(d-3)(d-4)\left(d^{2}-3 d+8\right)}{4\left(d^{2}-5 d+10\right)^{2}},
$$

which in the normalization $h_{I R}=1$ is reproducing eq. (3.48) of ref. [8]. Let us remind that the remaining constraints on the $f_{U V}$ and $\lambda h_{U V}$ are a simple consequence of the requirements $0<s_{U V}<(d-1) / 2$ and $s_{I R}<0$, that guarantee the existence of RG flow and its direction. They require that $L_{I R}$ must be the smallest physical scale:

$$
L_{U V}>L_{I R}, \quad 0<f_{U V}<f_{I R}, \quad 0<h_{U V}<h_{I R}, \quad W<0
$$

which reflects the properties of the critical points $W^{\prime}\left(\sigma_{c r}\right)=0$, namely that $\sigma_{U V}$ must be a maximum and $\sigma_{I R}$ the nearest local minimum of $W(\sigma)$.

The above discussion that leads us to the requirements (6.5), (6.6), (6.7) and (6.8) imposed on the $C F T_{U V / I R}$ data by the p.e.f. conditions (6.2), together with the arguments presented in Sect.4., can be summarized as follows:

The Holographic GB a/c Theorem statement - the central charges $a(l)$ and $c(l)$ are both positive and monotonically decreasing during the massless $R G$ flows - is valid for the $C F T_{U V / I R}$ satisfying the p.e.f. constraints as well, but only when the stronger p.e.f. restrictions on the physical scales $L_{U V / I R}$ and $\lambda$ take place. 
6.2.2. The $\frac{a}{c}$ and $t_{2}$ - Theorems. We next consider few consequences concerning the $\mathrm{RG}$ flows of the CFT's 3-point functions data. Instead of the $\mathcal{A} / \mathcal{B} / \mathcal{C}$-analogues of the Holographic $a / c$-Theorems we chose to study the $t_{2}(l)$ RG evolution ${ }^{38}$. The eqs. (4.1) and (4.7) allows us to rewrite the ratio of the central charges (for $c \neq 0$ ) in the following form:

$$
\frac{a}{c}=\frac{d-2}{d-4}-\frac{2}{(d-4) \sqrt{1-4 \lambda h}}
$$

Then the corresponding $\frac{a}{c}$-Theorems are a straightforward consequence of the RG flow conditions $0<h_{U V}<h_{I R}<h_{e p}$ :

$$
\text { - } \lambda>0: \quad \frac{a_{U V}}{c_{U V}} \geq \frac{a_{I R}}{c_{I R}} \quad \bullet \lambda<0: \quad \frac{a_{U V}}{c_{U V}} \leq \frac{a_{I R}}{c_{I R}},
$$

which is in fact a statement about the RG properties of $t_{2}$ : it is increasing for positive $\lambda$ and decreasing for negative $\lambda$. An alternative proof of the $t_{2}$-Theorem is based on its explicit form in terms of the $f$-variable:

$$
t_{2}(f, \lambda)=\frac{4 \lambda f(d-1)(d-2)}{(1-2 \lambda f)(d-3)(d-4)}, \quad \frac{d t_{2}}{d f}=\frac{4 \lambda(d-1)(d-2)}{(1-2 \lambda f)^{2}(d-3)(d-4)}
$$

and it again leads to the same conclusions.

Few comments concerning the eventual physical $(d=5)$ applications of the above results are now in order:

- On the $R G$ evolution of $E_{p}$. The holographic $t_{2}$-Theorem, together with the explicit forms (6.2) of the corresponding energy fluxes (with $t_{4}=0$ ), allows us to make conclusions about the RG evolution of these energy fluxes as well:

$$
E_{s}(U V)<E_{s}(I R), \quad E_{v}(U V)>E_{v}(I R) \quad E_{t}(U V)>E_{t}(I R) .
$$

Notice however the important difference between the $a$ and $c$ central charges and the $t_{2}$ and the $E_{p}$ 's $(\mathrm{p}=\mathrm{s}, \mathrm{v}, \mathrm{t})$ properties. Namely, the eqs. (4.1) represent the off-critical $Q F T_{d-1}$ definitions of the central functions $a(l)=a(f(\sigma))$ and $c(l)=c(f(\sigma))$ based on the holographic $\beta_{W}(\sigma)$-function, while the corresponding formulas determining $t_{2}$ and $E_{p}$ in terms of $a$ and $c$ (or as functions of $f$ ) have only well established critical meaning, i.e. they are valid for the $C F T_{U V / I R}$ only. The corresponding off-critical expressions for $t_{2}(\sigma)$ and $E_{p}(\sigma)$ can be derived by the conformal perturbations theory. It is expected that the critical values $t_{2}(U V / I R)$ and $E_{p}(U V / I R)$ receive relevant "bulk" contributions from the non-vanishing trace $\Theta\left(\sigma, x_{i}\right)=\beta(\sigma) \Phi_{\sigma}\left(x_{i}\right)$ of the stress-tensor, thus involving the structure constants of the following 3-point functions of $\Theta(\vec{x})$ and $T_{i j}(\vec{x})$ :

$$
<T_{i j}(\vec{x}) \Theta(\vec{y}) T_{k n}(0)>, \quad<\Theta(\vec{x}) \Theta(\vec{y}) \Theta(0)>, \quad<\Theta(\vec{x}) T_{i j}(\vec{y}) \Theta(0)>.
$$

They can be easily calculated within the frameworks of the $C F T_{U V}$ by applying the OPE's, discussed in Sect.2.:

$$
C_{\Theta \Theta \Theta}=\beta^{3}(\sigma) g(\sigma) C_{\Phi \Phi \Phi}, \quad C_{\Theta T \Theta}=\Delta_{\Phi}(\sigma) \beta^{2}(\sigma) g(\sigma),
$$

\footnotetext{
${ }^{38}$ We recall that in the GB case all the stress-tensor " 3 -points data" $\mathcal{A}, \mathcal{B}$ and $\mathcal{C}$ are well known functions of the two central charges $a \neq c \neq 0$.
} 
where $\Delta_{\Phi}(\sigma)$ is the anomalous dimension of the relevant operator $\Phi_{\sigma}(x)$, the $C_{\Phi \Phi \Phi}$ is the $\Phi_{\sigma}(x) \Phi_{\sigma}(0)$ OPE's structure constant, given by eq. $(2.20)$, and $g(\sigma)=C_{\Phi}^{(2)}$ is the 2-point function normalization constant (2.28). Notice that the above integrated 3-point functions involving $\Theta$ give relevant contributions to the off-critical bulk viscosity $\zeta(\sigma) \neq 0$ as well [63].

Although the derivation of the off-critical positive energy fluxes conditions is an open problem, the above established $t_{2}$ and $E_{p}$-Theorems contain an important information relating the UV- to IR- critical values of the corresponding quantities. However they have a quite different status and can not be considered as statements determining the monotonic properties of the yet unknown off-critical expression for $t_{2}(\sigma)$ and $E_{p}(\sigma)$ during the massless RG flows.

- $\eta / s$-Theorem? Let us remind that one of the main motivations for studying the $C F T^{\prime} s$ and, related to them, non-conformal $Q F T$ 's, dual to certain $d=5$ GB Gravity solutions - black holes, shock waves, DW's etc. - is their use in the description of the hydrodynamics of the strong coupled quark-gluon plasma [7, 16, 24-26]. As we have mentioned in the Introduction, the practical question to be answered concerns the possible violations of the "universal lower bound" $\eta / s=1 / 4 \pi$ of the ratio of shear viscosity to entropy density. The calculations based on the GB black hole solutions (duals to finite temperature CFT's) lead to the following simple form of this ratio in terms of the corresponding central charges $[8,9,11]$ :

$$
\left(\frac{\eta}{s}\right)_{U V} \approx \frac{1}{4 \pi}\left(\frac{a}{c}\right)_{U V}=\frac{1}{4 \pi}\left(3-\frac{2}{\sqrt{1-4 \lambda h_{U V}}}\right),
$$

which suggests that a similar formula is valid for the IR values of $\left(\frac{\eta}{s}\right)_{I R}$ as well. Although the considered RG flows interpolate between zero temperature $C F T_{U V / I R}$ 's, one might expect to have decreasing $\eta / s$ for positive $\lambda$, just as the above established $\frac{a}{c}$-Theorem is claiming. One argument in favour of such conclusion is that both the zero and finite temperature $C F T$ 's share the same central charges and they do satisfy the same p.e.f. conditions [15]. Another argument comes from the fact that the finite temperature $T=$ $1 / \beta_{\text {thg }}$ analogues of the considered DW's called "thermal gas" solutions ${ }^{39}$, are successfully used in the description of certain finite $T Q C D_{4}$-like and $s Q G P$ models up to certain not very high temperatures $T \in\left(0, T_{\min }\right)$, see for example refs.[71] and the references therein. The complete description of the off-critical behaviour of $\eta / s$ as a function of the RG scale (or of the coupling constant $\sigma$ ) by using or not the Holographic RG methods is however an open problem, out of the scope of the present paper.

\subsection{New minimal scales in $d=5$ QT Gravity $a / c$-Theorems}

The critical UV holographic features of $d=5$ Quasi-Topological Gravity (2.1) without matter interactions, together with the off-critical properties of the $a(l)$ central function, have been established in refs. [7]. Due to the fact that both $t_{2}$ and $t_{4}$ are now different

\footnotetext{
${ }^{39}$ they have the same form as the zero temperature DW's [5], but with the 4-d Minkowski part replaced by a circle $S_{1}$ of radius $\beta_{t h g}=1 / T$ for the time and by 3 -d torus for the space $x_{i}$-part.
} 
from zero, the p.e.f. conditions (6.2) are restricting their values within the triangle formed by the three curves $E_{s}\left(t_{2}, t_{4}\right)=0, E_{v}\left(t_{2}, t_{4}\right)=0$ and $E_{t}\left(t_{2}, t_{4}\right)=0$. We are interested in the new minimal scales arising from the p.e.f. requirements and imposing further stronger restrictions on the values of $f_{U V / I R}$. Our starting point are the following $d=5$ holographic expressions for $t_{2}$ and $t_{4}$ :

$$
t_{2}\left(f_{c r}\right)=\frac{24 f_{c r}\left(\lambda-87 \mu f_{c r}\right)}{1-2 \lambda f_{c r}-3 \mu f_{c r}^{2}}, \quad t_{4}\left(f_{c r}\right)=\frac{3780 \mu f_{c r}^{2}}{1-2 \lambda f_{c r}-3 \mu f_{c r}^{2}}, \quad f_{c r}=f_{U V / I R},(6.1
$$

derived in sect.4 of ref. [7]. We next substitute eqs. (6.12) into eqs. (6.2) and as a results we get the following consequences of the p.e.f. conditions for $f_{c r}$ :

$$
1-10 \lambda f+189 \mu f^{2} \geq 0, \quad 1+2 \lambda f-855 \mu f^{2} \geq 0, \quad 1+6 \lambda f+1317 \mu f^{2} \geq 0,(6 .
$$

where the positivity of the $c$ - central charge has been already taken into account. Let us denotes the roots of the above quadratic forms as

$$
\begin{aligned}
& f_{ \pm}^{e p, 1}=\frac{5}{189 \mu}\left(\lambda \mp \sqrt{\lambda^{2}-\frac{189}{25} \mu}\right), \\
& f_{ \pm}^{e p, 2}=\frac{1}{855 \mu}\left(\lambda \mp \sqrt{\lambda^{2}+855 \mu}\right), \\
& f_{ \pm}^{e p, 3}=-\frac{3}{439 \mu}\left(\lambda \mp \sqrt{\lambda^{2}-\frac{439}{3} \mu}\right),
\end{aligned}
$$

which in the case when they are real and positive introduce new p.e.f. scales $L_{ \pm}^{e p, k}=$ $L / \sqrt{f_{ \pm}^{e p, k}}$ with $k=1,2,3$.

In order to implement the p.e.f. requirements in the context of the $d=5 \mathrm{a} / \mathrm{c}$-Theorems (as stated in sect.3.) we need to know few properties of these $f_{ \pm}^{e p, k}\left(\lambda, \mu_{ \pm}\right)$, namely: (i) the intervals of values of $\lambda$, where the corresponding roots $f_{ \pm}^{e p, k}$ are real and positive for each one of the models $\mu_{+}$and $\mu_{-}$separately;(ii) which is the smallest one $f_{\min }^{e p}$ in between all these $f_{ \pm}^{e p, k}>0$ 's within each of the $\lambda$-regions established above; (iii) whether these $f_{\text {min }}^{e p}$ 's are smaller or bigger then the minimal of the other $a$-, top- and $c^{\prime}$ - scales $f_{p m}^{\eta}>0$ already used in the proof of the Theorem. The methods to perform this analysis are the same as those introduced in sect.3.2 and as always the graphical one is the most efficient. In the case when the smallest of these new p.e.f. scales turn out to be smaller then the "old ones" $f_{ \pm}^{\eta}$, the validity of the $a / c$-Theorem compatible with the $U V / I R$ - p.e.f. conditions imposes stronger restrictions on the allowed $f_{U V / I R}$ values: $0<f_{U V}<f_{I R}<f_{m i n}^{e p}$.

6.3.1.The $\mu_{+}$model. As one can see from the graphics of the functions $f_{ \pm}^{e p, k}(\lambda,+)$ plotted on Figs.(4(a))-(4(d)) and (5(a))-(5(c)), the $f_{ \pm}^{e p, 1} \in R$ only within the intervals

$$
-\infty<\lambda \leq 13.7427 \quad \text { and } \quad-2.235528<\lambda<\frac{1}{3}
$$

while the $f_{ \pm}^{e p, 2}$ are real only for $\lambda \in(-\infty, 0.250146)$, and the $f_{ \pm}^{e p, 3} \in R$ are real only when

$$
-\infty<\lambda<-3270.17 \quad \text { and } \quad 0.249152<\lambda<\frac{1}{3} \text {. }
$$


Their ordering in the regions where they are positive is clearly demonstrated by the corresponding graphics. For example, within the interval $\lambda \in(-\infty,-3520.09)$ we conclude by comparing the six curves presented on fig.(4(a)) that

$$
f_{+}^{e p, 1}<f_{+}^{e p, 2}<f_{-}^{e p, 1}<0<f_{-}^{e p, 3}<f_{-}^{e p, 2}<f_{+}^{e p, 3}
$$

and therefore the the minimal scale in this interval is given by $f_{-}^{e p, 3}$.

Analysing the existence of "critical" $\lambda$-points, where two of the curves $f_{ \pm}^{e p, k}$ are crossing each other, as shown on the Figs.(4(a))-(4(d), we realize that the $\lambda$-interval ( $-3520.09,0.239759)$ can be divided in five different subintervals, corresponding to different $f_{ \pm}^{e p, k}$, s orderings:

$$
\begin{array}{ll}
--3520.09<\lambda<-3270.17: & f_{+}^{e p, 1}<f_{+}^{e p, 2}<f_{-}^{e p, 1}<0<f_{-}^{e p, 2}<f_{-}^{e p, 3}<f_{+}^{e p, 3}, \\
\text { - }-3270.17<\lambda<-25.9505: & f_{+}^{e p, 1}<f_{+}^{e p, 2}<f_{-}^{e p, 1}<0<f_{-}^{e p, 2}, \\
\text { - }-25.9505<\lambda<-13.7427: & f_{+}^{e p, 1}<f_{-}^{e p, 1}<f_{+}^{e p, 2}<0<f_{-}^{e p, 2}, \\
\text { - }-13.7427<\lambda<0.235528: & f_{+}^{e p, 2}<0<f_{-}^{e p, 2}, \\
\text { - } 0.235528<\lambda<0.239759: & f_{+}^{e p, 2}<0<f_{-}^{e p, 2}<f_{+}^{e p, 1}<f_{-}^{e p, 1},
\end{array}
$$

Notice also that some of the $f_{ \pm}^{e p, k}(\lambda,+)$ 's that are real within one of these subintervals become complex in others ones ${ }^{40}$. The common feature of all these cases is that the smallest scale is always given by $f_{-}^{e p, 2}$.

As one can see from the plots on Figs.(4(d))-(5(b)), similar phenomena take place in the next interval $\lambda \in(0.239759,0.278864)$ :

- $0.239759<\lambda<0.249152: \quad f_{+}^{e p, 2}<0<f_{+}^{e p, 1}<f_{-}^{e p, 2}<f_{-}^{e p, 1}$,

- $0.249152<\lambda<1 / 4: \quad f_{-}^{e p, 3}<f_{+}^{e p, 2}<f_{+}^{e p, 3}<0<f_{+}^{e p, 1}<f_{-}^{e p, 2}<f_{-}^{e p, 1}$,

- $1 / 4<\lambda<0.250146$ : $\quad f_{-}^{e p, 1}<f_{-}^{e p, 2}<f_{+}^{e p, 2}<f_{+}^{e p, 3}<0<f_{+}^{e p, 1}<f_{-}^{e p, 3}$,

- $0.250146<\lambda<0.278864: f_{-}^{e p, 1}<f_{+}^{e p, 3}<0<f_{+}^{e p, 1}<f_{-}^{e p, 3}$,

where the minimal scale turns out to be $f_{+}^{e p, 1}$. Finally, as one can see from Fig.(5(c)) in the last interval we have

$$
\text { - } 0.278864<\lambda<1 / 3: \quad f_{-}^{e p, 1}<f_{+}^{e p, 3}<0<f_{-}^{e p, 3}<f_{+}^{e p, 1},
$$

Therefore now we can recognize $f_{-}^{e p, 3}$ as representing the minimal scale.

Notice the importance of the special points $\lambda_{c r}^{i, j}$ of the intersection of two of the curves $f \pm^{e p, i}$ and $f_{ \pm}^{e p, j}$, say at $\lambda_{c r}^{2,3}=-3520.09$ we have that $f_{-}^{e p, 2}=f_{-}^{e p, 3}$, etc. They are determining the limiting points of the $\lambda$ intervals, where at least one of the $f^{e p, k}$ is positive. The comparison (both the analytical and the graphical ones) of the minimal of the $f_{ \pm}^{e p, k}(\lambda+)>0$ 's with the minimal of the other $f_{ \pm}^{\eta}$ 's scales, within the above established "physically allowed" regions of $\lambda$, demonstrates that the p.e.f. scales are always smaller then all of the $f_{ \pm}^{\eta}$ scales. However in the different intervals defined by the corresponding

\footnotetext{
${ }^{40}$ the curves corresponding to complex values of $f_{ \pm}^{e p, k}$, in fact, do not appear on the figures.
} 


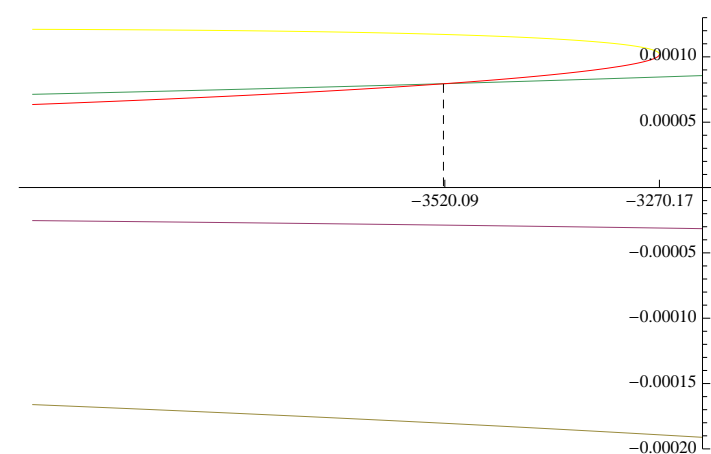

(a)

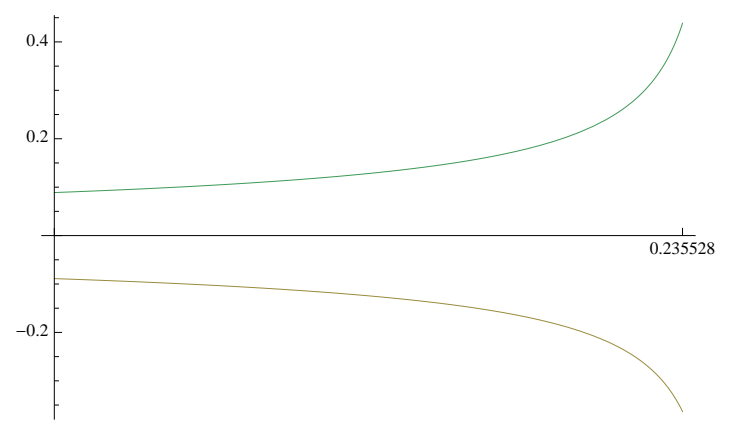

(c)

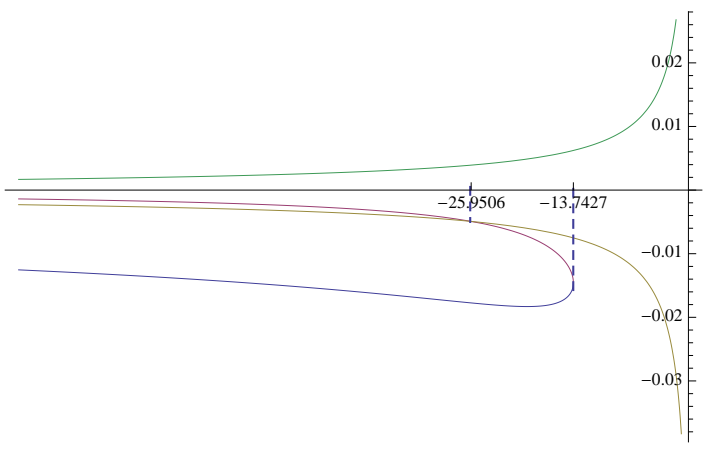

(b)

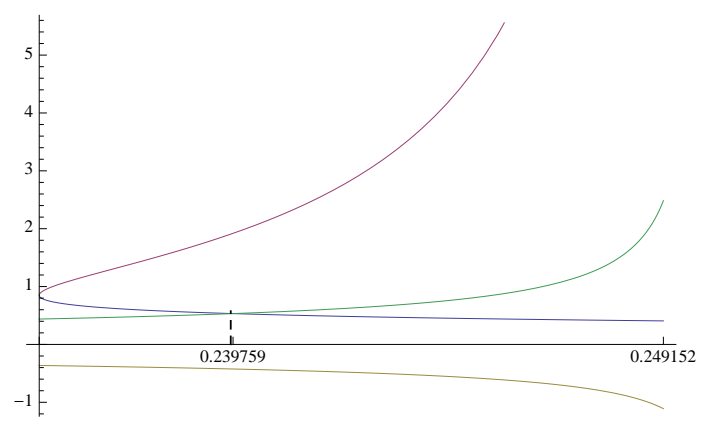

(d)

Figure 4. The $f_{ \pm}^{e p, i}\left(\lambda ; \mu_{+}\right)$curves for $\lambda \in(-\infty, 0.249152): f_{+}^{e p, 1}$ is depicted in blue; $f_{-}^{e p, 1}$ in purple; $f_{+}^{e p, 2}$ in beige; $f_{-}^{e p, 2}$ in green; $f_{+}^{e p, 3}$ in yellow and $f_{-}^{e p, 3}$ in red. The $\lambda$ values are within different intervals: on Fig. (a) $-\infty<\lambda<-3270.17$ (the $f_{+}^{e p, 1}$ is missed being too negative, namely $f_{+}^{e p, 1}<f_{+}^{e p, 2}<0$ ); on Fig.(b), $-3270.17<\lambda<0$; on Fig.(c), $0<\lambda<0.235538$ and on Fig.(d), $0.235538<\lambda<0.249152$.

$\lambda_{c r}^{i, j}$ we can have different minimal p.e.f. scales. Finally, the stronger form of the $d=5 a / c$ Theorems for the $\mu_{+}$model, with the p.e.f. requirements implemented, takes the following form:

$$
\begin{array}{llc}
\bullet & -\infty<\lambda<-3520.09 \quad\left(f_{-}^{e p, 2}=f_{-}^{e p, 3}\right), \quad f_{I R}<f_{-}^{e p, 3}, \\
\text { - } & -3520.09<\lambda<0.239759 \quad\left(f_{-}^{e p, 2}=f_{+}^{e p, 1}\right), & f_{I R}<f_{-}^{e p, 2}, \\
\text { - } & 0.239759<\lambda<0.278864 \quad\left(f_{+}^{e p, 1}=f_{-}^{e p, 3}\right), & f_{I R}<f_{+}^{e p, 1}, \\
\text { - } & 0.278864<\lambda<1 / 3, & f_{I R}<f_{-}^{e p, 3} .
\end{array}
$$

As we have shown in Sects.3 and 6.2., the Theorem can be also formulated as a set of restrictions on the allowed values of IR-scale $L_{I R}$, as for example

$$
L_{I R}>L_{-}^{e p, 3}, \quad \text { or } \quad L_{I R}>L_{-}^{e p, 2} \quad \text { or } \quad L_{I R}>L_{+}^{e p, 1} .
$$

6.3.2. The $\mu_{-}$model. Similar analysis based on the plots of the $f_{ \pm}^{e p, k}\left(\lambda, \mu_{-}\right)$curves presented on Fig.(6), demonstrate that in this case the $f_{ \pm}^{e p, 2}$ are complex, both the $f_{+}^{e p, 1}$ and $f_{+}^{e p, 3}$ are negative and the following ordering takes place:

$$
f_{+}^{e p, 1}<f_{+}^{e p, 3}<0<f_{-}^{e p, 3}<f_{-}^{e p, 1}<f_{+}^{a}
$$




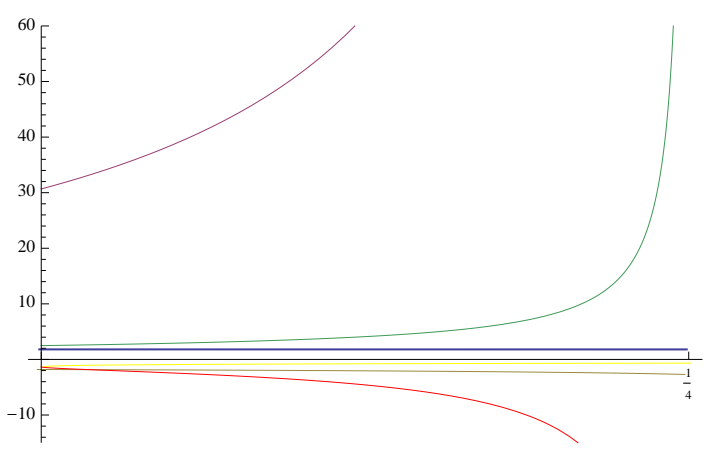

(a)

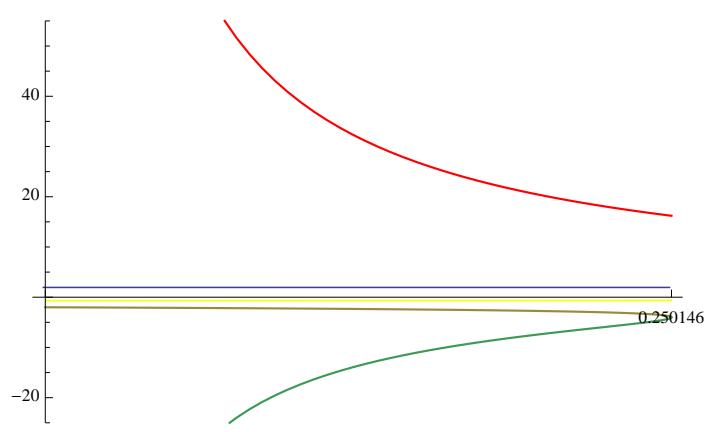

(b)

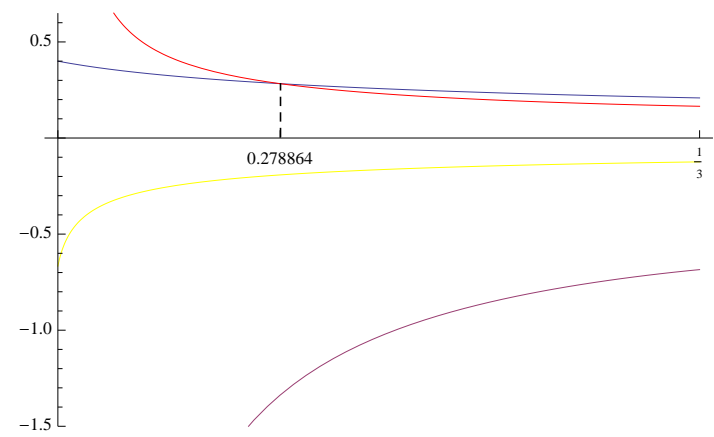

(c)

Figure 5. The $f_{ \pm}^{e p, i}\left(\lambda ; \mu_{+}\right)$curves for $\lambda \in(0.249152,1 / 3): f_{+}^{e p, 1}$ is depicted in blue; $f_{-}^{e p, 1}$ in purple; $f_{+}^{e p, 2}$ in beige; $f_{-}^{e p, 2}$ in green; $f_{+}^{e p, 3}$ in yellow and $f_{-}^{e p, 3}$ in red. The $\lambda$ values on different figures belong to different intervals: on Fig.( $a$ ), $0.249152<\lambda<1 / 4$; on Fig.(b), $1 / 4<\lambda<0.250146$ (here the "most negative" curve $f_{-}^{e p, 1}$ is missing again) and on Fig.(c), $0.250146<\lambda<1 / 3$.

Then the stronger form of the $a / c$-Theorems reads:

$$
f_{I R}<f_{-}^{e p, 3}, \forall \lambda<1 / 3 .
$$

6.3.3. On the $\lambda$ restrictions. An important remark concerns the additional p.e.f. restrictions on the $\lambda$ values similar to the ones of the GB models (6.8). We have to remind that in the considered case of QT Gravity we are working with the fundamental scale $L$ always fixed to be equal to the bare topological scale $L=L_{-}^{0 t o p}$, i.e $h_{-}^{\text {top }}=1$. The proper definitions of the $\mu_{ \pm}$models, i.e. the explicit form of the $\mu_{ \pm}(\lambda)$-functions, and correspondingly the allowed ranges of values for $\lambda$ (see sect.2.1.) are with respect to this particular choice of $L^{2}$. It is different from the normalization $h_{U V}=1$ used in ref. [7], where the p.e.f. conditions (6.13) lead to specific additional restrictions on $\lambda$, displayed on fig.1. of ref. [7]. In our topological normalization the same arguments as those of [7] - namely, solving the vacua equation $h_{I R}=f_{I R}\left(1-\lambda f_{I R}-\mu f_{I R}^{2}\right)$ and next replacing the corresponding solutions $f_{I R}^{(k)}\left(\lambda, \mu_{ \pm}\right)$into eqs. (6.13) - result in restrictions on $\lambda h_{I R}$ and not on the $\lambda$ alone. Hence once the choice $h_{-}^{\text {top }}=1$ is made, the p.e.f. requirements are imposing in fact further restrictions on the allowed values of $h_{I R}<h_{\max }$ and on the $h_{U V}<h_{I R}$ as well. Let us emphasise once more that the different normalizations $h^{\text {top }}=1$, 


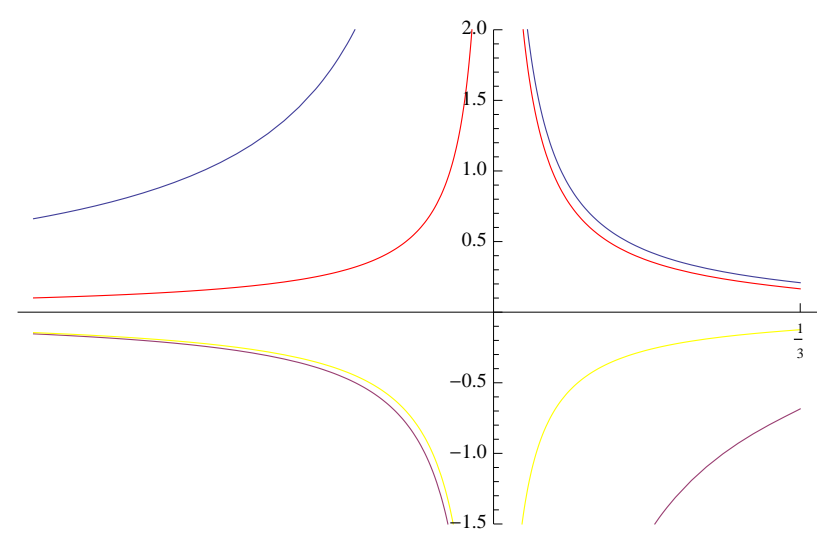

Figure 6. The curves $f_{ \pm}^{e p, i}\left(\lambda ; \mu_{-}\right): f_{+}^{e p, 1}$ is depicted in blue, $f_{-}^{e p, 1}$ in purple, $f_{+}^{e p, 3}$ in yellow and $f_{-}^{e p, 3}$ in red.

$h_{U V}=1$ and $h_{I R}=1$ although imposing different restrictions on $\lambda$ and $\mu$, are in fact equivalent.

Let us also mention that the implementation of the p.e.f. requirements into $d \geq 7$ $a / c$-Theorems for the $Q F T_{d-1}$ duals of QT Gravity is an open problem. It requires further holographic calculations of the explicit forms of the $t_{2}(f, \lambda, \mu)$ and $t_{4}(f, \lambda, \mu)$, which are yet unknown.

6.3.4. Towards the holographic $t_{4}$-Theorems. The fact that all the available "2- and 3-points" $C F T_{d-1}$ data: $a, c, t_{2}$ and $t_{4},{ }^{41}$ are holographically parametrized by the $f_{U V / I R}$ values, together with the off-critical identification $f(\sigma) \propto W^{2}(\sigma)$ of $f(\sigma)$ with the matter superpotential $W(\sigma)$, allows to establish the RG evolution of all the $C F T$ 's characteristics. Similarly to the GB case studied in Sect.6.2., we can derive the analogues of the $a / c$ Theorems for this new data. The simplest consequence concerns the RG evolution of the ratio $\frac{a}{c}$ which, together with the $t_{4}(f, \lambda, \mu)$, determines the properties of $t_{2}$ and of all the energy fluxes $E_{p}(f)$ as well. Considering its derivative :

$$
\frac{d}{d f}\left(\frac{a}{c}\right)=\frac{4\left(-\lambda+6 \mu f-9 \lambda \mu f^{2}\right)}{\left(1-2 \lambda f-3 \mu f^{2}\right)^{2}}
$$

we conclude that its sign is governed by the corresponding numerator. Instead of the exhaustive list of all the possibilities for the different signs of $\lambda$ and $\mu$ and of the roots $\tilde{f}^{ \pm}$ of the eq. $\left(-\lambda+6 \mu f-9 \lambda \mu f^{2}=0\right)$, we give here few representative examples:

$$
\begin{array}{ll}
\text { - } \mu>0, & \lambda<0:\left(\frac{a}{c}\right)_{U V}<\left(\frac{a}{c}\right)_{I R} \\
\text { - } \mu<0, \quad \lambda>0:\left(\frac{a}{c}\right)_{U V}>\left(\frac{a}{c}\right)_{I R} \text { for } f_{I R}<\frac{1+\sqrt{1+\frac{\lambda^{2}}{|\mu|}}}{3 \lambda}
\end{array}
$$

\footnotetext{
${ }^{41}$ and related to them energy fluxes $E_{p}\left(t_{2}, t_{4}\right)$ and the structure constants $\mathcal{A}, \mathcal{B}, \mathcal{C}$ and $C_{\Theta \Theta \Theta}$ as well.
} 
The complete description of the RG evolution of $t_{4}(f(\sigma))$ requires certain new holographic calculation to be performed by the conformal perturbation theory methods or/and by using the linear fluctuations around the QT Gravity DW's solutions. As we have already mentioned in sect.6.2 in the case concerning the " $t_{2}(\sigma)$-Theorem", it is expected that the $t_{4}(\sigma)$ in the dual non-conformal $Q F T_{4}$ will get new contributions from the off-critical terms related to the non-vanishing trace $\Theta\left(\sigma, x_{i}\right)=\beta(\sigma) \Phi\left(x_{i}\right)$ of the stress-tensor. Nevertheless the knowledge of $t_{4}(U V / I R)$ given by eqs. (6.12), together with the UV-to-IR flow condition $f_{U V}<f_{I R}$, allows us to derive the following relations between its critical values:

$$
\begin{aligned}
& \bullet \mu>0, \quad \lambda<0: t_{4}(U V)<t_{4}(I R), \quad \text { - } \mu>0, \quad \lambda>0: t_{4}(U V)<t_{4}(I R) \quad \text { for } \quad f_{I R}<\frac{1}{\lambda}, \\
& \text { - } \mu<0, \quad \lambda>0: t_{4}(U V)>t_{4}(I R) \quad \text { for } \quad f_{I R}<\frac{1}{\lambda}, \quad \bullet \mu<0, \quad \lambda<0: t_{4}(U V)>t_{4}(I R)
\end{aligned}
$$

The eventual monotonic properties of $t_{4}(\sigma)$ during the massless RG flow remain indeed an open problem.

\section{Holographic RG Flows and Phase Transitions}

The off-critical (a)AdS $S_{d} / Q F T_{d-1}$ correspondence, based on the d-dimensional Lovelocklike generalizations of the Einstein Gravity coupled to scalar matter with an appropriate superpotential $W<0$, is expected to provide non-perturbative solutions for certain $a \neq c$ unitary $Q F T_{d-1}$ 's in case the conditions of the $a / c$-Theorem and the positive energy fluxes requirements are fulfilled. The problem addressed in the present section concerns the description of the phase structure of such dual $Q F T_{d-1}$ 's, by applying the Holographic RG methods $[2,3]$ to the case of the cubic Quasi-Topological Gravity $[5,9,17,18]$.

Let us define it more precisely: Given a superpotential $W(\sigma)<0$ with few extrema, to deduce from the geometric data -vacua and flat DW's solutions [5] - the corresponding QFT-data: the characteristics of the CFT's representing UV and IR critical points, the RG evolution of this data, the specific properties of the massless and massive phases and the nature of the phase transitions between them. Although the rules of the off-critical version of the $A d S_{d} / Q F T_{d-1}$ correspondence, reviewed in Sect.7.1. below, are indeed universal and provide a consistent description of the QFT dual to the QT Gravity models independently on the particular form of the superpotential $W<0$, we choose to study the simplest representative example of quartic Higgs-like superpotential. As it is demonstrated in sects.7.2, 7.3 and 7.4. below, the corresponding dual $Q F T_{d}$ 's turns out to have interesting and rather realistic off-critical behaviour.

\subsection{On the Off-Critical Holography Dictionary}

7.1.1. $R G$ equations. Let us briefly recall the standard Wilson $\mathrm{RG}$ methods ${ }^{42}$ we are going to use in the description of the changes in the behaviour of the effective action and of the reduced free energy $F(\sigma) \sim e^{(d-1) l}$, of the correlation length $\xi(\sigma) \sim e^{-l}$ and of certain

\footnotetext{
${ }^{42}$ see for example the excellent textbooks [33], [35]
} 
correlation functions at the neighbourhood of each critical point $\sigma_{k}^{*}$. The scaling properties of their singular parts are determined by the well known RG equations:

$$
\begin{aligned}
& \beta(\sigma) \frac{d F_{s}(\sigma)}{d \sigma}+(d-1) F_{s}(\sigma)=0, \quad \beta(\sigma) \frac{d \xi(\sigma)}{d \sigma}=\xi(\sigma), \\
& \left|x_{12}\right| \frac{\partial G_{\Phi}\left(x_{12}, \sigma\right)}{\partial\left|x_{12}\right|}+\beta(\sigma) \frac{\partial G_{\Phi}\left(x_{12}, \sigma\right)}{\partial \sigma}+2\left(d-1+\frac{d \beta(\sigma)}{d \sigma}\right) G_{\Phi}\left(x_{12}, \sigma\right)=0
\end{aligned}
$$

whose solutions can be easily found for each given $\beta$-function. In the case of simple zeros $n_{k}=1$ of $\beta(\sigma)$, we can use its linear "near-critical" approximation $\beta(\sigma) \approx-s_{k}\left(\sigma-\sigma_{k}^{*}\right)$, which together with eq.(2.8) provide the explicit expressions (2.9) for the exact values of the critical exponents $s_{k}=-\frac{d \beta}{d \sigma}\left(\sigma_{k}^{*}\right) \neq 0$ of the dual $Q F T_{d}$. It is straightforward to check that at this approximation the RG equations (7.1) do reproduce the well known second order phase transitions scaling laws:

$$
\begin{aligned}
G_{\Phi}^{(k)}\left(x_{12}, \sigma\right)=<\Phi_{\sigma}\left(x_{1}\right) \Phi_{\sigma}\left(x_{2}\right)>_{k} & \approx \frac{e^{-\frac{\left|x_{12}\right|}{\xi_{k}}}}{\left|x_{12}\right|^{2\left(d-1-s_{k}\right)}} \\
F^{(k)}(\sigma) & \approx\left(\sigma-\sigma_{k}^{*}\right)^{\frac{d-1}{s_{k}}}, \quad \xi_{k} \approx\left(\sigma-\sigma_{k}^{*}\right)^{-\frac{1}{s_{k}}},
\end{aligned}
$$

at the neighbourhood of each critical point $\sigma_{k}^{*}$. The "higher order" zeros $n_{k}>1$ correspond to qualitatively different critical "scaling" behaviours, characterized by the specific essential singularities (involving the new parameters $\rho_{k}\left(n_{k}\right)$ ) of the correlation length and of the free energy:

$$
F^{(k)}\left(\sigma, n_{k}\right) \approx \exp \left[(d-1) \rho_{k}\left(n_{k}\right)\left(\sigma-\sigma_{k}^{*}\right)^{1-n_{k}}\right]
$$

known to describe infinite order phase transitions.

7.1.2. Basic Rules of the Off-Critical (a)AdS $S_{d} / Q F T_{d-1}$ correspondence. We next remind the basic ingredients and some of the particular features of the $a \neq c$ non-conformal Holography with a special emphasis on the restrictions that the a/c-Theorems requirements impose on the phase structure of the duals $Q F T_{d-1}$ :

(1) CFT data. Those of the stable extrema $\sigma_{k}^{c r}$ of the superpotential $W(\sigma)$ that represent simple zeros of the $\beta_{W}$-function (2.8), correspond to second order phase transitions. At such critical values $\sigma_{k}^{c r}$ of the coupling, the considered dual $Q F T_{d-1}$ becomes conformal invariant and its critical behaviour is described by a set of unitary $C F T_{d-1}^{(k)}=\left(c_{k}, a_{k}, \Delta_{k}, C_{\Delta}^{(k)}, t_{2}, t_{4}\right)$, duals to the stable physical vacua of the Quasi-Topological Gravity. Both the $C F T_{d-1}(U V)$ and $C F T_{d-1}(I R)$ related by the massless RG flows are constrained to satisfy all the unitarity and p.e.f. conditions (2.16) and (6.2).

(2) Massive and Massless $R G$ flows. The non-constant solutions $\sigma_{k, k+1}(l)=\sigma\left(l ; \sigma_{k}, \sigma_{k+1}\right)$ of the RG eqs.(2.8), representing the way the coupling constant $\sigma(l)$ of the dual $Q F T$ is running between two consecutive critical points, describe the RG flow and the phase transition that occurs at the UV-critical point $\sigma_{k}=\sigma_{U V}$. The same solution when inverted, i.e. $l(\sigma)=-\int \frac{d \sigma}{\beta(\sigma)}$ reproduces the DW's scale factor $e^{-2 l}=e^{2 A(\sigma)}$. In the case of polynomial 
superpotentials of $N$ extrema $\sigma_{k}$, the explicit solutions ${ }^{43}[5]$, representing $\xi(\sigma)$ and $F(\sigma)$ can be easily found from eqs. (7.1):

$$
\xi(\sigma)=G_{W}(\sigma) \prod_{k=1}^{N}\left(\frac{\sigma-\sigma_{k}}{\sigma_{0}-\sigma_{k}}\right)^{-\frac{1}{s_{k}}}, \quad \sum_{k=1}^{n} \frac{1}{s_{k}}=0, \quad \sigma \in R
$$

where $G_{W}(\sigma)<\infty$ is a certain known non-singular function, the critical exponents $s_{k}$ are given by eqs. (2.9) and $\sigma_{0} \in\left(\sigma_{k}, \sigma_{k+1}\right)$ denotes the "RG -initial" value of the coupling $\sigma$.

The analytic form of the $\xi(\sigma)$ and $F(\sigma)^{44}$ for all the values of the coupling $\sigma \in R$, i.e. within all the physically allowed intervals $p_{k}=\left(\sigma_{k}, \sigma_{k+1}\right) \in R$ called phases, determine few distinct RG evolutions, identified as massive $p_{k}^{\text {mass }}$ or massless $p_{k}^{m l}$ phases. In the massless phase the RG scale $L_{r g}(l)$ increases from $L_{r g}^{U V}=0$ to $L_{r g}^{I R}=\infty$, i.e. $\sigma(l=-\infty)=\sigma_{U V}$ and $\sigma(l=\infty)=\sigma_{I R}$, while in the massive phase it is reaching some specific finite scale $L_{r g}^{\max }=1 / M_{m s}$ for infinite values of the coupling:

$$
\begin{aligned}
& \text { massless }(U V \rightarrow I R): 0<L_{r g} \leq \infty \quad \xi\left(\sigma_{U V}^{*}\right) \approx \infty, \quad \xi\left(\sigma_{I R}^{*}\right) \approx 0 ; \quad \sigma( \pm \infty)=\sigma_{I R / U V} \\
& \text { massive }(U V \rightarrow \infty): 0<L_{r g} \leq L_{r g}^{m s} \quad \xi\left(\sigma_{U V}^{*}\right) \approx \infty, \quad \xi(\sigma \approx \infty) \approx L_{r g}^{\max }
\end{aligned}
$$

The inverse $M_{m s}$ of the maximal scale $L_{r g}^{\max }$ defines the smallest mass gap in the energy spectrum and as a consequence of the eqs. (7.1) the corresponding 2-point correlation function manifest exponential decay $e^{-M_{m s}\left|x_{12}\right|}$, typical for the IR limit of the free massive particle propagator. This behaviour has to be compared to the one of the massless $R G$ flows, where in order to reach the maximal coupling's distance $\left|\sigma_{I R}-\sigma_{U V}\right|$ the scale $l$ must run over the entire interval $L_{r g} \in(0, \infty)$.

The massless phases are geometrically described by the $A d S_{d}\left(L_{U V}\right) / A d S_{d}\left(L_{I R}\right)$ DW's, with the UV-critical point representing its $A d S_{d}(U V)$ boundary. Instead in the massive phases $p^{\text {mass }}=\left(\sigma_{U V}, \infty\right)$, the running coupling $\sigma(l)$ gets its maximal value for a finite RG distance $L_{r g}^{\max }$ corresponding to $\sigma\left(L_{r g}^{\max }\right)=\infty$. Such behaviour is known to have a geometrical description in terms of the singular DW's of $A d S_{d} /$ n.s.-type, whose boundary is again related to certain UV vacuum, but its IR region terminates at a naked singularity $y=y_{0}$, i.e. $R\left(y_{0}\right)=-\infty$. Observe that both the regular and the singular DW's are described by the same scale factor $e^{2 A}(\sigma) \sim \xi^{2}$ as in eq. (7.4) above, considered in different intervals of values of $\sigma$. The finite value of the correlation length $\xi(\sigma=\infty) \sim 1 / M_{m s}$ in the massive phase is a consequence of the special property of the critical exponents: $\sum_{k=1}^{n} \frac{1}{s_{k}}=0$ (see ref. [5]).

We should also mention that for polynomial superpotentials $W_{N}(\sigma)$ with $N \geq 2$, the GB and the QTG Holographic RG flows and those based on the pure EH gravitymater models are of quite a different nature. Namely, the EH's induced $\beta_{E H}$-function has $\sigma_{n s}^{c r}=\infty$ as a degenerate critical point, i.e. $\beta_{E H}(\infty)=0$ with $s_{n s}=0=c_{n s}^{E H}$, which corresponds to the EH's solutions of naked singularity ${ }^{45}$. Such a problem is absent in the

\footnotetext{
${ }^{43}$ for marginally degenerated critical points $s_{U V}=0$, i.e. for second or higher order zeros of $\beta$, new terms containing essential singularities appear, as shown in sect.4.3. of ref. [5].

${ }^{44}$ as well as of the corresponding 1- and 2-point correlation functions $<\Phi_{\sigma}\left(x_{i}\right)>$ and $<\Phi_{\sigma}\left(x_{i}\right) \Phi_{\sigma}\left(y_{j}\right)>$

${ }^{45} \mathrm{As}$ is well known it has not an appropriate $A d S / C F T$ description since the large cosmological constants, i.e. CFT's of small and zero central charges, are out of the validity of the AdS/CFT correspondence [1]
} 
considered GB- and QTG-matter models, due to the fact that now we have always that $\beta_{G B / Q T G}(\infty)=\infty$. Hence the naked singularity is not a critical point anymore, but an ending point of the massive phase of the dual QFT.

(3) Phase Transitions. When the superpotential has few extrema, we can order the positions $\sigma_{k}$ of all the physical and topological vacua, that give rise to a set of consecutive intervals $\left(\sigma_{k}, \sigma_{k+1}\right), k=1,2, \ldots, n$, whose ends determine the b.c.'s of a specific DW. These DWs solutions define a finite "chain" of consecutive DW's of common boundaries and/or horizons $[5,34]$. Each one of the individual $D W_{k, k+1}$ 's of this chain is characterized by the specific "initial" (in $y$ ) value $\sigma(0)=\sigma_{0} \in\left(\sigma_{k}, \sigma_{k+1}\right)$ of the matter field, that serves as a coupling constant initial condition $\sigma_{0}$ for the RG eqs. (2.8) and (7.4). For different superpotentials and for different values of $\lambda$ and $\mu$ we can have distinct sequences of DW's corresponding to qualitatively different (a)AdS geometries: the stable ones $A d S_{d}(I R) / A d S_{d}(U V)$, the singular ones $A d S_{d}(U V) / n . s .$, etc. In the dual $Q F T$ such DW chain represents a set of different massive and massless phases defining its phase structure. The change of the IR -type of b.c.'s that occurs at each one of the boundaries represents the transition between two different type of $(a) A d S_{d}$ geometries: for example the simplest two DWs chain $A d S_{d}(I R) / A d S_{d}(U V) / n . s$. describes the massless-to-massive second order phase transition, etc.

The above discussion makes evident that the complete phase structure of the dual $Q F T_{d-1}$ can be described by few different perturbed $C F T_{d-1}\left(\sigma_{U V}^{k}\right)$ 's $(1.2)$ - as many as different UV critical points we have. Therefore in order to establish the validity of the offcritical (a)AdS $S_{d} / Q F T_{d-1}$ correspondence in a region of the coupling space including one critical point $\sigma_{U V}^{k}$ it is sufficient to find certain perturbed $C F T_{d-1}$, whose $C F T$ data and near-critical behaviour of the $\Phi_{\sigma}$ correlation functions coincide with the ones derived from the Quasi-Topological Gravity-matter model with an appropriate superpotential $W(\sigma)$. Notice that the non-perturbative $\beta$-function (2.8) contains all the information about the complete phase structure and on the nature of the phase transitions for all the allowed values of the coupling $\sigma$, but indeed in the specific for $A d S / C F T$ large UV-scales $L_{U V} \gg 1$ ( i.e. $N_{c}^{2} \gg 1$ ) approximation.

\subsection{Quartic Higgs-like Superpotential}

There is a wide variety of superpotentials $W(\sigma)$ that lead to physically consistent Holographic RG flows and interesting phase structures of the corresponding dual $Q F T_{d}$ 's. We next consider the simplest representative example of the quartic superpotential $W(\sigma)=$ $-B\left[\left(\sigma^{2}-x_{0}\right)^{2}+D\right]$ of inverted "double-well" type. For $B, D$ and $x_{0}$ all positive, i.e. for $W(\sigma)<0$, it allows an explicit analytic construction of the physical DWs ${ }^{46}$ (see sect.4 of ref. [5] for details).

The extrema $W^{\prime}(\sigma)=0$ of the superpotential for $\sigma \geq 0$ denoted by $\sigma_{\mathrm{IR}}=0$ and $\sigma_{\mathrm{UV}}=\sqrt{x_{0}}$ are candidates for representing the physical vacua ${ }^{47}$ :

$$
W_{\mathrm{IR}}=-B\left(x_{0}^{2}+D\right), \quad f_{\mathrm{IR}}=\frac{L^{2} B^{2}\left(x_{0}^{2}+D\right)^{2}}{(d-2)^{2}} ; \quad W_{\mathrm{UV}}=-B D, \quad f_{\mathrm{UV}}=\frac{L^{2} B^{2} D^{2}}{(d-2)^{2}},
$$

\footnotetext{
${ }^{46}$ Due to the reflection symmetry $W(-\sigma)=W(\sigma)$, we restrict our analysis to the case $\sigma>0$ only.

${ }^{47}$ in this section and in the App. B we are fixing for simplicity $\kappa=1$.
} 
where $W_{\mathrm{IR}}=W\left(\sigma_{\mathrm{IR}}\right)$, etc. and $f_{\mathrm{IR}}=L^{2} / L_{\mathrm{IR}}^{2}$. Here $L^{2}$ is given by the normalization established in Sect.3., i.e. $L^{2}=L_{0 t o p}^{2}$. Therefore we can easily relate $B, D$ and $x_{0}$ to the corresponding vacua scales:

$$
B x_{0}^{2}=\frac{(d-2)}{L}\left(\sqrt{f_{\mathrm{IR}}}-\sqrt{f_{\mathrm{UV}}}\right), \quad B D=\frac{(d-2)}{L} \sqrt{f_{\mathrm{UV}}},
$$

As a result the $a / c$-Theorem and p.e.f. requirements on the $f_{U V / I R}$ (derived in Sects.3-6) are now easily transformed into certain conditions on the parameters of $W(\sigma)$. Further restrictions on these parameters are imposed by the BF unitarity condition and by the requirement that the operators $\Phi_{\sigma}^{U V}\left(x_{i}\right)$, driving the RG flows, are relevant, namely that $0<s_{U V}<d-1$.

As we have demonstrated in Sect.2.1., the complete vacua structure of the considered models also includes the topological vacua and depending on the signs of the GB and Lovelock couplings $\lambda$ and $\mu$ we can have few of them ${ }^{48}$. Their "positions" $\sigma_{\text {top }}^{ \pm}$are given by all the real solutions of the following equation:

$$
\left(\frac{\left(\sigma_{\text {top }}^{ \pm}\right)^{2}}{x_{0}}-1\right)^{2}=\frac{\sqrt{f_{0}^{t o p}}-\sqrt{f_{\mathrm{UV}}}}{\sqrt{f_{\mathrm{IR}}}-\sqrt{f_{\mathrm{UV}}}}
$$

where $f_{0}^{\text {top }}$ denotes $f_{G B}^{\text {top }}=\frac{1}{2 \lambda}$ for the GB case and the corresponding $f_{ \pm}^{\text {top }}$ for the cubic QT gravity models (see sect.2.1 above). The sign of the denominator is the sign of $B$, hence in order that the r.h.s. of the last equation to be positive, i.e. $B>0$, the conditions $f_{\mathrm{UV}}<f_{\mathrm{IR}}$ and $f_{\mathrm{UV}}<f_{ \pm}$have to be satisfied.

It is worthwhile to mention that the Holographic RG methods reviewed in this subsection are perfectly valid in the case of cubic Quasi-Topological Gravity-matter models of more complicated polynomial or non-polynomial Superpotentials [3, 38], as well as when the matter sector involves more then one scalar fields $[3,76]$. The explicit form of the corresponding DW's scale factors for other choices of the few extrema superpotential, as for example the super-gravity induced one $W(\sigma)=B \cosh (\kappa \sigma)[2 \delta-\cosh (\kappa \sigma)]$ and of the periodic one $W=B \cos (\kappa \sigma)-D$, share many of the properties of DW's of the "doublewell" superpotential, studied here. The relevance of the quadratic superpotential must be pointed out. As is well known, the construction of the corresponding DW's [5] allows to describe the near-critical behaviour of the $Q F T_{d-1}$ dual to GB or QT Gravity models, based on arbitrary matter superpotentials.

\subsection{Phase Transitions in QFT's duals to GB Gravity}

According to the Holographic RG rules, the main features of the massless and massive RG flows in the QFT's dual to GB Gravity with quartic Superpotential [5], can be extracted from the analytic properties of the DW's scale factor (see sect.4.4. of ref. [5]) or equivalently

\footnotetext{
${ }^{48}$ in the case of the QT Gravity for $\mu>0$ we have only one such vacua, two - for $\mu<0$ and $0<\lambda<1 / 3$ and no one when both $\lambda$ and $\mu$ are negative.
} 
from the zeros and the singularities of the correlation length in the dual model:

$$
\begin{aligned}
& \xi(\sigma) \equiv e^{A(\sigma)}=e^{A_{\infty}}\left(\sigma^{2}\right)^{-1 / 2 s_{\mathrm{IR}}}\left|\sigma^{2}-x_{0}\right|^{-1 / s_{\mathrm{UV}}} G_{\text {top }}(\sigma), \quad G_{\text {top }}=\prod_{j=1}^{4}\left|\sigma^{2}-\sigma_{j}^{2}\right|^{-1 / s_{\text {top }}^{j},} \\
& s_{\mathrm{UV}}=16 B x_{0} L_{\mathrm{UV}}\left(1-L_{\text {top }}^{2} / L_{\mathrm{UV}}^{2}\right) ; s_{\mathrm{IR}}=-8 B x_{0} L_{\mathrm{IR}}\left(1-L_{\text {top }}^{2} / L_{\mathrm{IR}}^{2}\right) ; \\
& s_{\text {top }}^{j}=-64(d-2)^{-1} B^{2} x_{0}^{-2} L_{\text {top }}^{2}\left(\sigma_{j}^{2}-x_{0}\right)^{2} \sigma_{j}^{2} ; j=1, \ldots, 4 ;
\end{aligned}
$$

Following ref. [5], we have introduced the parameters $\sigma_{j}^{2} \equiv u_{j}+x_{0}$ with $u_{1}=-u_{2}=$ $u_{+} ; u_{3}=-u_{4}=\tilde{u}_{+}$given by:

$$
u_{+}=\sqrt{D} \sqrt{\frac{L_{U V}}{L_{t o p}}-1} ; \quad \tilde{u}_{+}=i \sqrt{D} \sqrt{\frac{L_{U V}}{L_{t o p}}+1},
$$

that are related to the positions of the "topological vacua", i.e. all the (real or/and complex numbers, depending on the values of $\lambda$ ) algebraic solutions of eqs. (7.6) above. We further parametrize the normalization constant $\exp \left(A_{\infty}\right) \equiv \frac{1}{M}$ in terms of the RG "initial value" $\sigma_{0}$ of the coupling $\sigma$ by choosing the standard Wilson RG normalization $\xi\left(\sigma_{0}\right)=1$.

The restrictions imposed by the GB $a / c$-Theorem on the DWs properties and equivalently on the nature of the corresponding $Q F T_{d-1}$ 's phases, strongly depend on the sign of $\lambda$. Therefore the description of the phase transitions in these two cases requires a separate discussion.

\subsubsection{Negative $\lambda$ models: massless-to-massive phase transition}

For negative values of $\lambda$ the requirement of positivity of both central functions $a>0$ and $c>0$ does not lead to any restrictions on the $f_{U V / I R}$ values. The p.e.f. requirement (6.7) however introduces a minimal scale $L_{-}^{e p}$ and it constrains $f_{U V / I R}$ 's into to the interval:

$$
0<f_{U V}<f_{I R}<\frac{(d-3)}{2|\lambda|(d+1)}
$$

Since all the $f_{G B}^{\eta}$, given by eq. (4.2), are now negative numbers, we have no topological vacua at all. Notice that the GB domain walls scale factors $\exp (2 A(\sigma)) \sim \xi^{2}(\sigma)$ are given again by eqs. (7.7), but with $L_{t o p}=L \sqrt{2 \lambda}$ replaced by $i\left|L_{t o p}\right|=i L \sqrt{2|\lambda|}{ }^{49}$. Although both $s_{\text {top }}^{j}$ and $\sigma_{j}^{2}$ become complex numbers (see App. B):

$$
\begin{aligned}
& \sigma_{1}^{2}=x_{0}+\sqrt{D} \sqrt{\frac{L_{U V}}{i L_{t o p}}-1}=\left(\sigma_{3}^{2}\right)^{*}=\left(\sigma_{1}^{2}\right)^{R}+i\left(\sigma_{1}^{2}\right)^{I}, \\
& \sigma_{2}^{2}=x_{0}-\sqrt{D} \sqrt{\frac{L_{U V}}{i L_{t o p}}-1}=\left(\sigma_{4}^{2}\right)^{*}=\left(\sigma_{2}^{2}\right)^{R}+i\left(\sigma_{2}^{2}\right)^{I}, \quad \sigma_{j}^{2}=\left(\sigma_{j+2}^{2}\right)^{*} \\
& s_{U V}=16 B x_{0} L_{U V}\left(1+\frac{L_{t o p}^{2}}{L_{U V}^{2}}\right), \quad s_{I R}=-8 B x_{0} L_{I R}\left(1+\frac{L_{t o p}^{2}}{L_{I R}^{2}}\right), \\
& s_{\text {top }}^{j}=\frac{64 B^{2} L_{\text {top }}^{2}}{(d-2)} \sigma_{j}^{2}\left(\sigma_{j}^{2}-x_{0}\right)^{2}=\left(s_{\text {top }}^{j+2}\right)^{*}=\left(s_{\text {top }}^{j}\right)^{R}+i\left(s_{\text {top }}^{j}\right)^{I}, j=1,2
\end{aligned}
$$

\footnotetext{
${ }^{49}$ in our normalization $L=L_{0 t o p}$.
} 
the function $G_{\text {top }}^{-}(\sigma)$ remains real and for negative $\lambda$ it has no singularities:

$$
\begin{aligned}
G_{\text {top }}^{-} & =\prod_{j=1}^{2}\left[\left(\sigma^{2}-\left(\sigma_{j}^{2}\right)^{R}\right)^{2}+\left(\left(\sigma_{j}^{2}\right)^{I}\right)^{2}\right]^{-\frac{s_{j}^{R}}{\left(s_{j}^{R}\right)^{2}+\left(s_{j}^{I}\right)^{2}}} \times \\
& \times \exp \left(\frac{2 s_{j}^{I}}{\left(s_{j}^{R}\right)^{2}+\left(s_{j}^{I}\right)^{2}} \arctan \left(\frac{\left(\sigma_{j}^{2}\right)^{I}}{\sigma^{2}-\left(\sigma_{j}^{2}\right)^{R}}\right)\right)
\end{aligned}
$$

The corresponding scale factor is an example of a specific chain $A d S_{d}(I R) / A d S_{d}(U V) / n . s$. of two DWs, that give rise to the following phase structure of the dual QFT:

- Massless phase. The standard $A d S_{d}(I R) / A d S_{d}(U V)$ - domain wall connects the two physical GB vacua $\sigma_{U V / I R}$. Their dual are unitary $C F T_{d-1}(U V / I R)$ 's, which are supposed to respect all the a/c-Theorem and p.e.f. requirements (see sect.6.2.). For positive values of $B$ and $x_{0}$, satisfying the condition:

$$
0<16 B x_{0} L_{\mathrm{UV}}\left(1+L_{\text {top }}^{2} / L_{\mathrm{UV}}^{2}\right)<\frac{(d-1)}{2},
$$

the operator $\Phi_{\sigma}^{U V}\left(x_{i}\right)$ of dimension $\Delta_{U V}=d-1-s_{U V}<d-1$ is relevant. As one can conclude from the explicit form (7.7) of the correlation length, the RG evolution from $C F T(U V)$ to $C F T(I R)$, i.e when $\sigma \in\left(\sigma_{I R}, \sigma_{U V}\right)$, describes the massless phase of the $Q F T_{d}$ dual to the GB-matter model for negative $\lambda$ within the interval (6.8).

- Massive phase. The singular DW of $A d S_{d}(U V) / n . s$-type defined within the interval $\left(\sigma_{U V}, \infty\right)$ shares the same boundary $A d S_{d}(U V)$ as the $A d S_{d}(I R) / A d S_{d}(U V)$ - domain wall. Hence the boundary conditions for the matter field $\sigma(y)$ of both DW's, determined by the UV-critical exponent $s_{U V}$, do coincide. They present however rather different behaviours at the deep IR-region: $\xi_{G B}\left(\sigma_{I R}\right)=0$ in the massless phase has to be compared to the following finite value of the correlation length:

$$
\xi_{G B}(\sigma \rightarrow \infty)=\frac{1}{M_{G B}}, \quad M_{G B}=\left(\sigma_{0}^{2}\right)^{-1 / 2 s_{\mathrm{IR}}}\left|\sigma_{0}^{2}-x_{0}\right|^{-1 / s_{\mathrm{UV}}} G_{\text {top }}^{-}\left(\sigma_{0}\right),
$$

with $\sigma_{0} \in\left(\sigma_{U V}, \infty\right)$, indicating the presence of the finite mass gap $M_{G B}\left(\lambda, L_{U V}, d, \sigma_{0}\right)$ in the energy spectrum of the dual $Q F T_{d-1}$. Therefore the interval $\left(\sigma_{U V}, \infty\right)$ can be identified as the massive phase of this $Q F T$. Notice the crucial role of the following important identity :

$$
\frac{1}{2 s_{I R}}+\frac{1}{s_{U V}}+2 \sum_{j=1}^{2} \frac{s_{j}^{R}}{\left(s_{j}^{R}\right)^{2}+\left(s_{j}^{I}\right)^{2}}=0
$$

in the derivation of the mass formula (7.12). The Holographic RG flows describing the evolution of the $C F T_{U V}$ data, i.e. the $a(\sigma) \neq c(\sigma)$ central functions and $\beta(\sigma)$, shown on fig.(7), demonstrate all the features typical of the second order massless-to-massive phase transition occurring at $\sigma=\sigma_{U V}$ in the dual $Q F T_{d-1}$.

- The dual $Q F T_{d-1}$ identification. The verification of the validity of the conjectured duality between GB domain walls and the RG flows of certain QFT consists in the comparison of the available perturbative QFT data with the Holographic RG data. As usually, the 


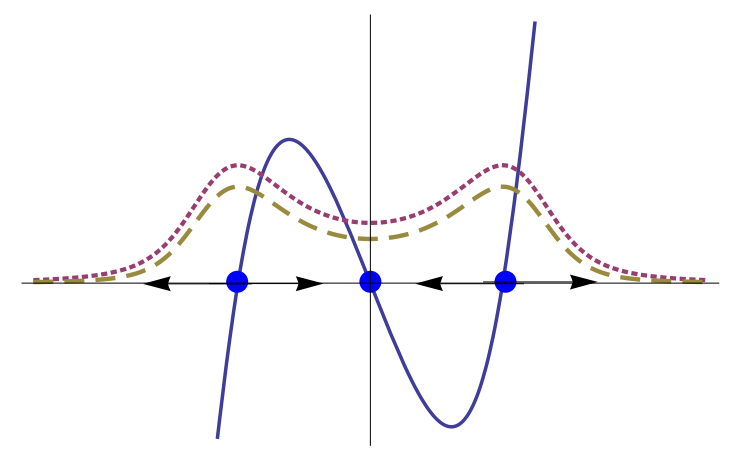

Figure 7. Massless-to-massive phase transition in $Q F T_{4}$ dual to GB model with $\lambda<0$ : $-\beta(\sigma)$-function is depicted in blue; $c(\sigma)$-function in beige and $a(\sigma)$-function in purple.

GB vacua solutions ${ }^{50}$ are recognized as certain $\left(\mathcal{N}=1\right.$ supersymmetric) $C F T_{d-1}$ 's defined by their central charges $c_{U V}, a_{U V}$ and by the relevant operator $(\mathrm{s}) \Phi_{\sigma}^{U V}\left(x_{i}\right)$ of dimension $\Delta_{U V}$, whose OPE's has the form (2.18) with structure constant:

$$
C_{\Phi \Phi \Phi}^{U V}=\frac{24(d-2) \Gamma((d-1) / 2)}{D \pi^{(d-1) / 2}} \sqrt{x_{0}}\left(1-\left(\frac{L_{+}^{t o p}}{L_{U V}}\right)^{2}\right) .
$$

The problem is to find an appropriate (super-symmetric) $C F T_{d-1}$, possessing all these specific geometrically induced properties. In the cases when such perturbative OPE's information is available, on can identify the non-conformal $Q F T_{d-1}$, having the phase structure established above, as certain perturbed CFT (1.2). The argument for such conclusion comes from the well known fact [33] that starting with such $\mathrm{p} C F T_{d-1}$ action, one can easily reproduce the perturbative $\beta(\tilde{\sigma})$-function (2.19) near to the UV-critical point $\sigma_{U V}$ with $\tilde{\sigma}=\sigma-\sigma_{U V}$ and $s^{+}=s$, as explained in Sect.2.2.3. The particular form of the solutions of the corresponding RG equations (2.8)

$$
\begin{aligned}
\tilde{\sigma}(l) & =\frac{s \sigma_{0} e^{s l}}{C_{\Delta}\left[\sigma_{0}\left(e^{s l}-1\right)+s / C_{\Delta}\right]}, \\
\xi(\tilde{\sigma}) & =\left(\frac{\tilde{\sigma}-\frac{s}{C_{\Delta}}}{\tilde{\sigma}}\right)^{1 / s}\left(\frac{\sigma_{0}}{\sigma_{0}-\frac{s}{C_{\Delta}}}\right)^{1 / s}, \quad \frac{1}{M}=\xi(\tilde{\sigma} \rightarrow \infty) \sim\left(\frac{\sigma_{0}-s / C_{\Delta}}{\sigma_{0}}\right)^{-1 / s}
\end{aligned}
$$

confirms that such pCFT (for small $\left|\sigma-\sigma_{U V}\right|<1$ ) indeed describes a second order phase transition almost identical to the one obtained from the holographic $\beta$-function (2.8). Notice that for the inverted Higss superpotential we are considering, the OPE's of the irrelevant $\Phi_{\sigma}^{I R}\left(x_{i}\right)$ operator of dimension $\Delta_{I R}$ (from the $C F T^{I R}$ representing IR critical point) are quite different from the UV-ones: the quadratic term in eq. (2.18) is now absent due to the vanishing of the IR-structure constant $C_{\Phi \Phi \Phi}^{I R}=0$.

\footnotetext{
${ }^{50}$ For large values of the corresponding $C F T$-central charges, i.e. $L_{U V} / l_{p l}>L_{I R} / l_{p l}>\left|L_{t o p}\right| / l_{p l} \gg 1$, where the $A d S / C F T$ correspondence is valid
} 
It is worthwhile to mention that the complete identification of the dual QFT's as certain $p C F T$ indeed requires further comparison between the corresponding $\Phi_{\sigma}\left(x_{i}\right)$ 's correlation functions, obtained from the analysis of small fluctuations around the DW's background in GB Gravity-matter model with the ones calculated by the conformal perturbations techniques.

\subsubsection{Positive $\lambda$ models: massless RG flows}

The GB Gravity-matter model in the case of positive GB-coupling $\lambda$ within the interval (6.8) permits few topological vacua $\sigma_{t o p}^{j}$ defined by the zeros of the c-function $c\left(f_{G B}^{t o p}\right)=0$. The condition $c \geq 0$ for causality (i.e. absence of ghosts) of the GB Gravity ${ }^{51}$ introduces a natural fundamental minimal scale $L_{G B}^{0 t o p}=L_{G B}^{t o p} / \sqrt{2 \lambda}$, which however is different form the smallest physical p.e.f. scale $L_{+}^{e p} \geq L_{G B}^{t o p}$, as shown in sect.6.2. The remaining a/c-Theorem and p.e.f. conditions are satisfied when the physical vacua scales $L_{U V / I R}$ are restricted as follows:

$$
L_{U V}>L_{I R} \geq L_{+}^{e p}=\frac{L_{G B}^{0 t o p}}{\sqrt{f_{+}^{e p}}}
$$

which has as a consequence that $s_{U V}$ is positive and all the others $s_{I R}$ and $s_{\text {top }}^{j}$ are negative. Then the DW's scale factor (7.7) describes (for $\sigma>0$ ) a "chain" of two different GB domain walls of common boundary: the physical one $A d S_{d}(I R) / A d S_{d}(U V)$ and "phystop" one $A d S_{d}(U V) / A d S_{d}(t o p)$, which is relating the physical UV vacua to the topological one $\sigma_{\text {top }}$. The behaviour of the correlation length (7.7), namely $\xi\left(\sigma_{U V}\right) \approx \infty$ and $\xi\left(\sigma_{I R}\right)=$ $0=\xi\left(\sigma_{\text {top }}\right)$, indicates that both DW's might describe massless $R G$ flows. However only the "physical vacua" phase $\sigma \in\left(\sigma_{I R}, \sigma_{U V}\right)$ satisfies all the $C F T(U V / I R)$ 's consistency conditions: positivity of the a-central function and the p.e.f.'s. Notice that the c-central function is positive and monotonically decreasing in both phases $\left(\sigma_{I R}, \sigma_{U V}\right)$ and $\left(\sigma_{U V}, \sigma_{\text {top }}\right)$.

We next realize that the $C F T_{\text {top }}$ is characterized by $c_{\text {top }}=0$ and $a_{\text {top }}<0$. Therefore the "phys-top" phase $\sigma \in\left(\sigma_{U V}, \sigma_{t o p}\right)$ is divided in two parts by the "critical point" $\sigma_{c r}^{a}$ with $a\left(\sigma_{c r}^{a}\right)=0$, which is reached when the RG scale gets its critical value $l_{c r}=L_{G B}^{a}$, as shown on Fig.(8(a)). The physically consistent part $\left(\sigma_{U V}, \sigma_{c r}^{a}\right)$ of this phase, where $a>0$ is still positive, thus represents a natural mass scale $M_{G B}^{a}=1 / L_{G B}^{a}$. The complete description of such eventually physical " $a$-massive" phases is out of the scope of the present paper. The degenerate case $L_{I R}=L_{+}^{a}$ of coinciding IR- and $a$-scales, demonstrated on Fig.(8(b)), is an example where the zero $\sigma_{c r}^{a}$ of $a(\sigma)$-central function is representing the IR critical point. However such values of $L_{I R}$ do not satisfy the GB p.e.f. conditions (6.7) and (7.14). Therefore the corresponding dual QFT's have to be discarded as physically inconsistent. Finally, in the example shown on Fig.(8(c)), i.e. for $L^{2} / f_{+}^{t o p}\left(\lambda ; \mu_{+}\right)<L_{I R}^{2}<L^{2} / f_{+}^{a}\left(\lambda ; \mu_{+}\right)$ the $a(\sigma)$ - central function changes its sign within the former massless phase $\left(\sigma_{U V}, \sigma_{I R}\right)$, thus violating the p.e.f. conditions and the $a / c$ - Theorem.

\footnotetext{
${ }^{51}$ and equivalently one of the requirements for the unitarity of the dual $C F T_{d-1}$
} 


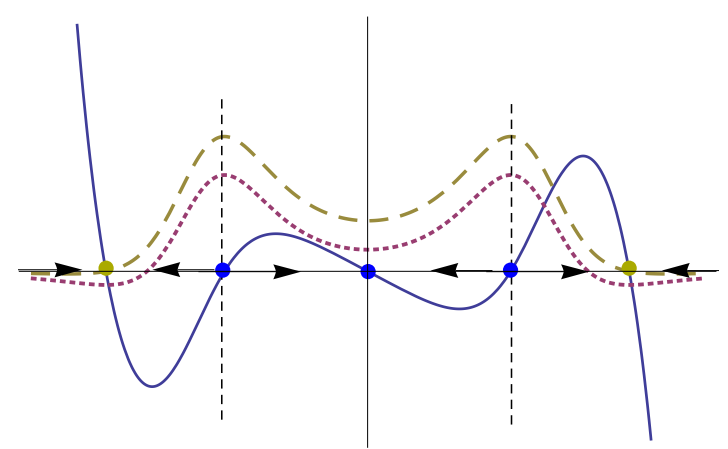

(a)

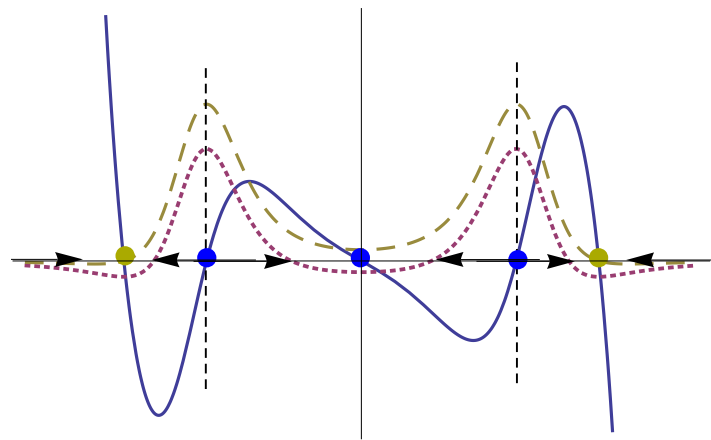

(c)

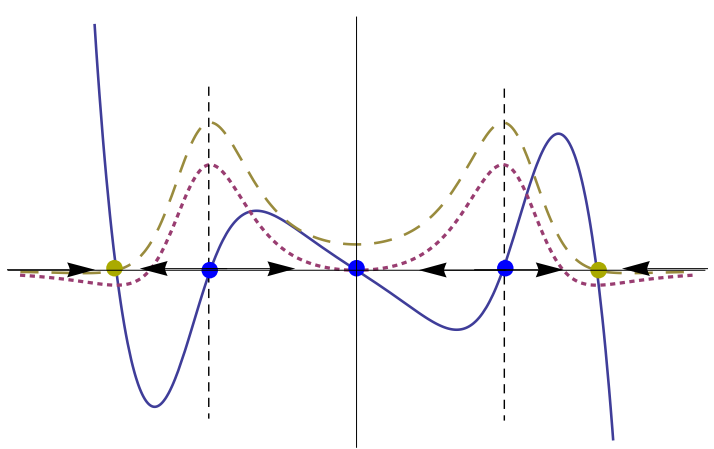

(b)

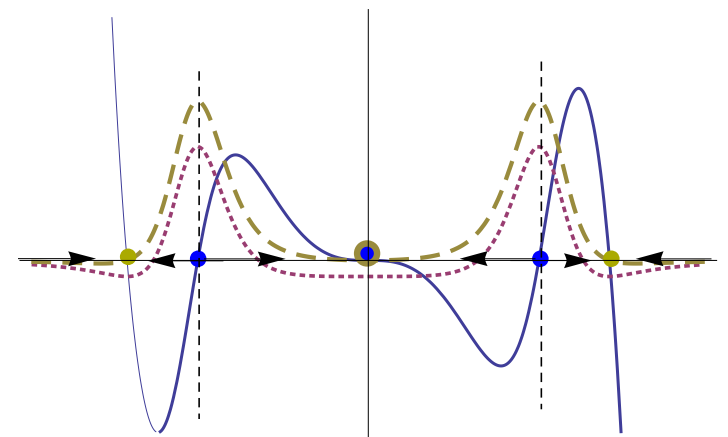

(d)

Figure 8. RG flows in $d=5 \mu_{+}$-model with $\lambda=\frac{1}{4}$ : the $-\beta(\sigma)$-function is the continues blue curve; the $c(\sigma)$-function is the beige traced one and the $a(\sigma)$-function is the purple doted curve; the critical points that are extrema of $W(\sigma)$ are depicted in blue, while the beige ones represent topological $c=0 C F T_{4}$ 's. The IR-scale $L_{I R}$ restrictions: on Fig.(a) $L_{I R}^{2}>L^{2} / f_{+}^{a}\left(\lambda ; \mu_{+}\right)$; on Fig.(b) $L_{I R}^{2}=L^{2} / f_{+}^{a}\left(\lambda ; \mu_{+}\right)$; on Fig.(c) $L^{2} / f_{+}^{t o p}\left(\lambda ; \mu_{+}\right)<L_{I R}^{2}<L^{2} / f_{+}^{a}\left(\lambda ; \mu_{+}\right)$and on Fig.(d) $L_{I R}^{2}=L^{2} / f_{+}^{t o p}\left(\lambda ; \mu_{+}\right)=L_{t o p}^{2}$.

- Marginal degenerations. Let us also mention the limiting case $L_{I R}=L_{G B}^{t o p}$, when the $\mathrm{UV}$-vacuum $\sigma_{U V}^{2}=x_{0}$ coincides with two of the topological vacua $\sigma_{j}^{2}=x_{0}$ for $j=1,2,{ }^{52}$. The scale factor and $\xi(\sigma)$ now exhibit an essential singularity at $\sigma^{2}=x_{0}$ :

$$
\xi(\sigma)=e^{A(\sigma)} \propto\left(\sigma^{2}\right)^{-\frac{1}{2 s_{a}^{(0)}}} \exp \left(-\frac{D}{32 x_{0} B L\left(\sigma^{2}-x_{0}\right)^{2}}\right) \prod_{i=3}^{4}\left|\sigma^{2}-x_{i}\right|^{-\frac{1}{s_{g r}^{i}}}
$$

Although it involves complex quantities, the last term can be rewritten in a real form similar to the one of eq. (7.11). This qualitatively new behaviour is known to be specific for the infinite order phase transitions, which is expected to take place in the dual $Q F T$. It can be confirmed also by perturbative RG calculations in the dual $Q F T$ with $s_{U V}=0$, similar to the ones presented at the end of the previous subsection. Notice however that in this particular example of marginally degenerated critical point, graphically represented on

\footnotetext{
${ }^{52}$ remember that $\sigma_{j}^{2}$ and $s_{\text {top }}^{j}$ for $j=3,4$ are complex conjugate of each other, as one can see from their definitions (7.8) for $\lambda>0$ and do not represent topological vacua at all
} 
Fig.(8(d)), the p.e.f. conditions (7.14) are violated. Hence it does not represent physically consistent unitary $Q F T_{d-1}$.

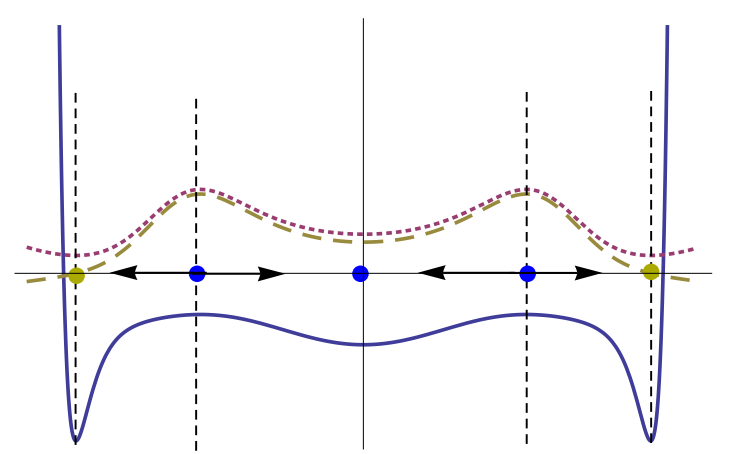

(a)

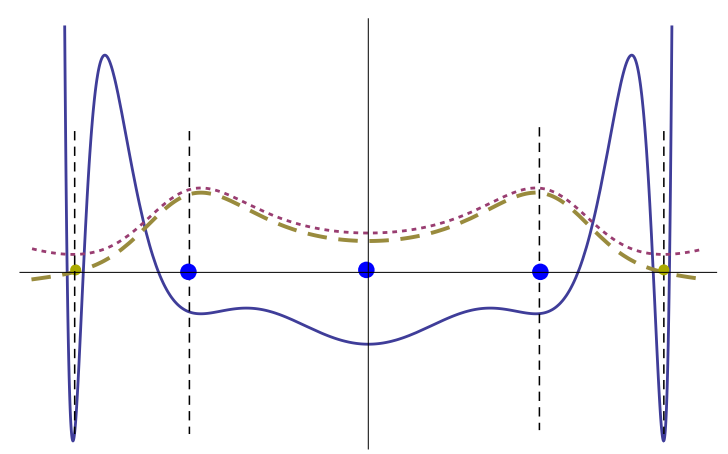

(b)

Figure 9. The matter potential $V(\sigma)$, in blue, for $d=5 \mu_{+}$-models with $\lambda<\frac{5}{27}$ and $L_{I R}^{2}>$ $L^{2} / f_{+}^{a}\left(\lambda ; \mu_{+}\right)$: on Fig.(a) $0<s_{U V}<4$, while on Fig.(b) $s_{U V}>4$. The $c(\sigma)$-function is the traced beige curve, while the $a(\sigma)$-function - the red dotted one.

- Comment about $s_{U V}>d-1$ case. As we have mentioned in sect.2.2.2 (see also sect.4.4 of ref. [5]), the effect of this new restriction on the superpotential parameter $B x_{0}$ (replacing the old one $s_{U V}<d-1$ ) is that the operator $\Phi_{\sigma}^{U V}\left(x_{i}\right)$ has now negative dimension $\Delta_{\Phi}<0$. Therefore the corresponding $C F T(U V)$ is non-unitary ${ }^{53}$. The main difference between these two cases is that the corresponding old $V_{(a)}(\sigma)$ and new $V_{(b)}(\sigma)$ matter potentials, shown on Figs. (9(a)) and (9(b)), have different number of extrema, although the inverted "double-well" form of the superpotentials $W_{(a)}$ and $W_{(b)}$ is preserved. The presence of two more "maxima" in the potential $V_{(b)}$ reflects the fact that together with $m_{I R}^{2}$ and $m_{\text {top }}^{2}$, also the $m_{U V}^{2}(b)$ is now positive. Hence the new maxima must appear in between the "neighbour" minima $\sigma_{U V}$ and $\sigma_{t o p}$ (in the left) and $\sigma_{I R}$ (in the right). Such GB domain walls are interpolating now between two minima of the matter potential $V_{(b)}$, while in the case of $V_{(a)}$ they are relating one maximum with the neighbouring minima.

\subsection{RG Flows from Quasi-Topological Gravity DW's}

The flat DW's solutions of the Quasi-Topological Gravity with inverted Higgs-like Superpotential in $d \geq 5$ dimensions, constructed in ref. [5], serve as a main tool in the analysis of the Holographic RG flows in the corresponding dual $Q F T_{d-1}$. Again the basic ingredients in the description of the massless and massive QFT's phases are the correlation length and the reduced free energy:

$$
\xi(\sigma) \propto e^{A(\sigma)}=e^{A_{\infty}}|\sigma|^{-1 / s_{\mathrm{IR}}}\left|\sigma^{2}-x_{0}\right|^{-1 / s_{\mathrm{UV}}} \prod_{j=1}^{8}\left|\sigma^{2}-\sigma_{j}^{2}\right|^{-1 / s_{t o p}^{j}},
$$

Similarly to the GB Gravity case, we have denoted the positions of the "topological vacua" $\sigma_{j}^{2} \equiv u_{j}+x_{0}$, with $x_{0}=\sigma_{U V}^{2}$, independently of whether they are real or/and complex

\footnotetext{
${ }^{53}$ another possibility is the so called $\Delta_{-}$-quantization [30] corresponding to spontaneous breaking of the conformal symmetry. Such DW's however do not describe RG flows in the dual $Q F T_{d-1}$.
} 
numbers. The parameters $u_{1}=-u_{2}=u_{+} ; \quad u_{3}=-u_{4}=\tilde{u}_{+} ; \quad u_{5}=-u_{6}=u \quad u_{7}=$ $-u_{8}=\tilde{u}_{-}$are defined as follows:

$$
u_{ \pm}=\sqrt{\frac{(d-2)}{B}} \sqrt{\frac{1}{L_{t o p}^{ \pm}}-\frac{1}{L_{U V}}} ; \quad \tilde{u}_{ \pm}=i \sqrt{\frac{(d-2)}{B}} \sqrt{\frac{1}{L_{t o p}^{ \pm}}+\frac{1}{L_{U V}}}
$$

The explicit values of the corresponding "critical exponents" $s_{k}(2.9)$ are given by:

$$
\begin{aligned}
& s_{\mathrm{UV}}=16 B x_{0} L_{\mathrm{UV}}\left(1-\frac{\left(L_{\text {top }}^{+}\right)^{2}}{L_{\mathrm{UV}}^{2}}\right)\left(1-\frac{\left(L_{\text {top }}^{-}\right)^{2}}{L_{\mathrm{UV}}^{2}}\right), \\
& s_{\mathrm{IR}}=-8 B x_{0} L_{\mathrm{IR}}\left(1-\frac{\left(L_{\text {top }}^{+}\right)^{2}}{L_{\mathrm{IR}}^{2}}\right)\left(1-\frac{\left(L_{\text {top }}^{-}\right)^{2}}{L_{\mathrm{IR}}^{2}}\right) \\
& s_{\text {top }}^{p}=-64(d-2)^{-1} B^{2} x_{0}^{-2}\left(L_{\text {top }}^{+}\right)^{2}\left(\sigma_{p}^{2}-x_{0}\right)^{2} \sigma_{p}^{2}\left(1-\frac{\left(L_{\text {top }}^{-}\right)^{2}}{\left(L_{\text {top }}^{+}\right)^{2}}\right) ; p=1, \ldots, 4 . \\
& s_{\text {top }}^{q}=-64(d-2)^{-1} B^{2} x_{0}^{-2}\left(L_{\text {top }}^{-}\right)^{2}\left(\sigma_{q}^{2}-x_{0}\right)^{2} \sigma_{q}^{2}\left(1-\frac{\left(L_{\text {top }}^{+}\right)^{2}}{\left(L_{\text {top }}^{-}\right)^{2}}\right) ; q=5, \ldots, 8 .
\end{aligned}
$$

According to their definition (see sect.2.2.2), they are related to the scaling dimensions $\Delta_{k}$ of certain dual conformal fields $\Phi_{\sigma_{k}}\left(x_{i}\right)$ from the conjectured $\mathrm{CFT}_{d-1}$ 's "attached" to each one of the vacua of the considered Quasi-Topological Gravity-matter model. The values of the corresponding OPE's structure constants (2.20) for these operators are given by:

$$
\begin{aligned}
C_{\Phi \Phi \Phi}^{U V} & =\frac{24(d-2) \Gamma((d-1) / 2)}{D \pi^{(d-1) / 2}} \sqrt{x_{0}} C_{0}^{U V}, \quad C_{\Phi \Phi \Phi}^{I R}=0=C_{\Phi \Phi \Phi}^{t o p} \\
C_{0}^{U V} & =1-2 \lambda\left(\frac{L_{+}^{0 t o p}}{L_{U V}}\right)^{2}-3 \mu\left(\frac{L_{+}^{0 t o p}}{L_{U V}}\right)^{4}
\end{aligned}
$$

They determine the singular part of the "holographic" OPE's (2.18) and are essential for the identification of the dual QFT's as appropriate perturbed CFT's (similarly to the GB case considered in Sect.7.3.1). By construction, the irrelevant operators $\Phi_{I R}^{\text {top }}$ for all the $C F T_{\text {top }}^{(j)}$ have vanishing $C_{\Phi \Phi \Phi}^{t o p}$, independently of the form of the superpotential, due to the fact that always $C_{0}\left(\sigma_{\text {top }}^{j}\right)=0$. The vanishing of the IR structure constant however is a specific property of the $Z_{2}$ symmetric form of the quartic superpotential under investigation. It turns out that the new superpotentials $\tilde{W}=W+K \sigma^{3}$ obtained by adding of a cubic term, give rise to dual $Q F T$ models having both $C_{\Phi \Phi \Phi}^{U V} \neq 0$ and $C_{\Phi \Phi \Phi}^{I R} \neq 0$ different from zero.

Observe that the scale factor $e^{2 A(\sigma)} \sim \xi^{2}(\sigma)$ given by eq. (7.16), for all the considered $d \geq 5$ QT Gravity models, represent two specific DWs chains: $A d S_{d}(I R) / A d S_{d}(U V) / n . s$. or the $A d S_{d}(I R) / A d S_{d}(U V) / A d S_{d}(t o p)$ one. Let us remind that the holographic description of such "two-phases structure" of the dual $Q F T_{d-1}$ 's, depends not only on the analytic properties of $\xi(\sigma)$, but also on the values and the sign of the $a(l)$ and $c(l)$-central functions. The holographic $a / c$-Theorem selects as physically admissible those of the phases, for which both $a(\sigma)$ and $c(\sigma)$ are positive for all the values $\sigma \in\left(\sigma_{I R}, \sigma_{U V}\right)$ and $\sigma \in\left(\sigma_{U V}, \sigma_{+}^{t o p}\right)$ or 
$\sigma \in\left(s_{U V}, \infty\right)$ and the corresponding critical values $f_{U V / I R}$ 's satisfy the p.e.f. conditions. The results of Sects.3-6 suggest that the extensive list of different QT Gravity models can be organized in the following three families of models of identical phase structures: (i) of minimal scale $f_{I R}<f_{+}^{a}$ or $f_{I R}<f_{+}^{\text {top }}$, (ii) of maximal scale $f_{U V}>f_{-}^{c^{\prime}}$ and (iii) no minimal or maximal $L_{ \pm}^{\eta}\left(\eta=\right.$ top $\left., a, c^{\prime}\right)$ scales at all.

\subsubsection{Minimal scale phase structures}

A common feature of all the $Q F T_{d-1}$ 's with minimal scale's phase structure, duals to the $\mu_{ \pm}$QTG models for different ranges ${ }^{54}$ of values of $\lambda$ is that they satisfy one of the following two type of restrictions:

(a) $f_{U V}<f_{I R}<f_{+}^{a}\left(\lambda ; \mu_{ \pm}\right)$defining the GB-like massless $\left(\sigma_{I R}, \sigma_{U V}\right)$ and a-massive $\left(\sigma_{U V}, \sigma_{c r}^{a}\right)$ phases;

(b) $f_{U V}<f_{I R}<f_{+}^{t o p}\left(\lambda ; \mu_{ \pm}\right)$leading to a new "top-massless" $\left(\sigma_{U V}, \sigma_{+}^{\text {top }}\right)$ phase, together with the standard $\left(\sigma_{I R}, \sigma_{U V}\right)$ massless one.

It is important to mention that the p.e.f. requirements discussed in Sect.6.3. in the case of $d=5$ QT Gravity models are in fact imposing stronger minimal scales restrictions (6.17) and (6.19):

$$
f_{U V}<f_{I R}<f_{ \pm}^{e p, k}<f_{+}^{a}<f_{+}^{t o p}
$$

where $f_{ \pm}^{e p, k}=L^{2} /\left(L_{ \pm}^{e p, k}\right)^{2}$ with $k=1,2,3$ denote one of the the minimal p.e.f. scales (6.16), say $f_{-}^{e p, 3}$, depending on the specific interval (6.17) of values of $\lambda$ under consideration. They determine the physically consistent massless phases, while in the case of massive phases they are imposing restrictions on the corresponding $f_{U V}$ 's values.

7.4.1. Phase structure of $Q F T_{4}$ 's dual to $d=5$ QT Gravity. The explicit analytic form of the correlation length (7.16) for the $Q F T_{4}$ 's dual to the $\mu_{ \pm}$QTG models for all the values of $\lambda \in(-\infty, 1 / 3)$ demonstrates that the $\left(x_{0}, 0\right)$-phase has all the features of a true massless phase. If we further restrict the critical values $f_{U V}=f\left(x_{0}\right)$ and $f_{I R}=f(0)$ to belong to one of p.e.f. allowed intervals (6.17) or (6.19), then it describes an unitary consistent massless RG flow in the dual $\mathrm{QFT}_{4}$.

Depending on the specific properties of the $a$-central function, we can realize two qualitatively different behaviours of the dual $Q F T_{4}$. We first consider the models of type (a):

$$
\text { - } \mu_{+}(d=5), \quad \lambda \in\left(\frac{5}{27}, \frac{1}{3}\right) ; \quad \quad \mu_{-}(d=5), \quad \lambda \in\left(-\infty, \frac{1}{3}\right)
$$

which are characterized by the existence of one zero $\sigma_{c r}^{a} \in\left(\sigma_{U V}, \sigma_{+}^{t o p}\right)$ of the $a$-central function, representing the end of the holographic description. As in the GB case, it introduces a specific new mass scale $M_{a}=1 / L_{+}^{a}$, which motivate us to consider the physically consistent part of this phase $\left(\sigma_{U V}, \sigma_{c r}^{a}\right)$ as an " $a$-massive phase". The Holographic RG flows of the consider type (a) models within the interval $\lambda \in(5 / 27,1 / 3)$ are practically identical to the ones of the GB case, which is graphically represented on Figs.(8(a))-(8(d)) for a particular GB value $\lambda=1 / 4$ of the $\mu_{+}(d=5)$ - model for $0<s_{U V}<4$.

\footnotetext{
${ }^{54}$ notice that the particular form of these $\lambda$-intervals is $d$-dependent, as shown in Sect.5 and App. A
} 


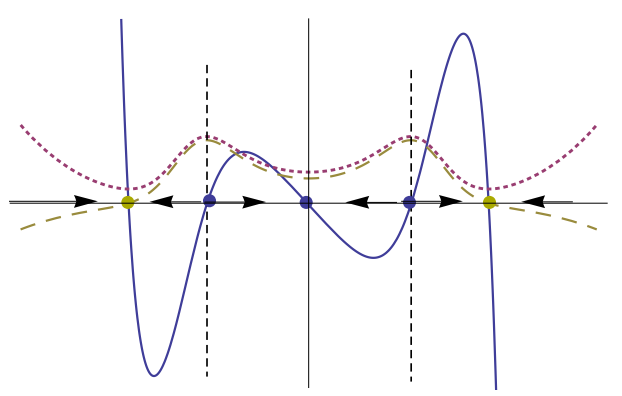

(a)

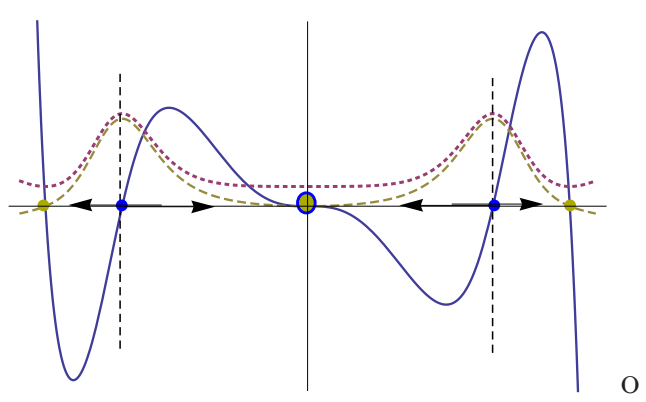

(b)

Figure 10. RG flows in $d=5 \mu_{+}$models with $\lambda<\frac{5}{27}$. The continues blue curve represents the $-\beta(\sigma)$-function, the $c(\sigma)$-function is the traced beige curve and the $a(\sigma)$-function is the red dotted one. The IR-scales restrictions: on Fig.(a) $L_{I R}^{2}>L^{2} / f_{+}^{t o p}\left(\lambda ; \mu_{+}\right)$and on Fig. (b) $L_{I R}^{2}=L^{2} / f_{+}^{t o p}$, representing a marginal degenerated critical point.

While the holographic properties as well as the unitarity and causality of the massless phase $\left(\sigma_{U V}, \sigma_{I R}\right)$ are well established, the complete understanding of this " $a$-massive" phase and of its physical consistency requires further investigations of the corresponding off-critical $<\Phi\left(x_{i}\right) \Phi(0)>$ correlation functions. Similarly, the identification of the second order phase transition at $\sigma_{U V}=x_{0}$ as being of massless-to- $a$-massive type needs more then the Holographic RG arguments we have used. Namely, the calculations of the offcritical mass spectrum by considering the small fluctuations around the considered DW background are required.

We next consider the models of type $(b): \mu_{+}(d=5, \lambda)$ with $\lambda \in\left(-\infty, \frac{5}{27}\right)$, whose physically consistent massless phase $\left(x_{0}, 0\right)$ coincides with the one of the type (a) models described above. The main difference is that now $a(\sigma)$ is positive over the entire interval $\left(\sigma_{U V}, \sigma_{t o p}^{+}\right)$that represents a new "top-massless" phase as indicated by the behaviour of the $\xi$ : namely, $\xi\left(\sigma_{U V}\right)=\infty$ and $\xi\left(\sigma_{\text {top }}^{+}\right)=0$. The corresponding massless-to-top-massless second order phases transition is graphically demonstrated on Fig.(10(a)) for the case of $L_{I R}>L_{+}^{t o p}$. The plots presented on Fig.(10(b)) describe the RG flows in the degenerate case $L_{I R}=L_{+}^{\text {top }}$, where the IR vacua $\sigma_{I R}=\sigma_{\text {top }}$ (the beige round central dot ) coincides with one of the topological vacua, giving rise to a particular second order phase transition between two "top-massless" phases.

Notice that independently of the positivity of both central charges $a$ and $c$ during the "physical-to-top- massless" RG flow, the "top-massless" phase is not satisfying the p.e.f conditions (6.17). Therefore it does not represent consistent unitary and causal $Q_{F T}$. Although the proof of the causal inconsistency or of the eventual bulk instabilities of the corresponding $A d S_{d}(U V) / A d S_{d}(t o p)$ DW's is still missing, the field-theoretical p.e.f.'s type of arguments are sufficient to classify the $d=5$ models of type (b) as physically inconsistent.

The above discussion leads us to the following conclusion concerning the dual $Q F T_{4}$ 's off-critical properties:

Assuming the validity of the Holographic Renormalization Group for the cubic QT 
Gravity-matter models ${ }^{55}$ and imposing the a/c-Theorem and p.e.f. requirements, no standard massive phases of $\xi(\sigma \rightarrow \infty)=1 / M$ can be realized. Hence the corresponding physically consistent dual $Q F T_{4}$ 's contain only one well defined massless phase $\left(\sigma_{U V}, \sigma_{I R}\right)$.

7.4.2. Comments on the phase structure of QFT's dual to $d \geq 7$ models. The properties of the chains of DW's solutions and a part of the corresponding $a / c$-Theorem requirements, corresponding to the $d \geq 7$ models satisfying the minimal scale restrictions, turns out to be almost identical to the $d=5$ case considered above. In what follows we shall give the complete list of these $d \geq 7$ models with a brief summary of their main features, emphasizing the few important differences with the $d=5$ models.

- Massless and a-Massive phases. The $d \geq 7$ Quasi-Topological Gravity models, whose dual $Q F T_{d-1}$ 's are of type (a), i.e. representing massless and $a$-massive phases are given by:

$$
\begin{aligned}
& \text { - } \mu_{+}(d \geq 7),-\infty<\lambda<\frac{d(d-4)}{3(d-2)^{2}}, \quad \quad \mu_{-}(d=7,8), \lambda \in\left(0, \frac{1}{3}\right) \\
& \text { - } \mu_{-}(d \geq 9), 0<\lambda<\frac{(d-8)(d-4)}{3(d-6)^{2}}
\end{aligned}
$$

Again the explicit expression of their correlation lengths (7.16) confirms this phase structure. Notice that when one or two of the topological scales $L_{ \pm}^{\text {top }}$ become imaginary, as in the case of $\mu_{-}(d=5)$ model with $\lambda \in(-\infty, 0)$ and also for the $\mu_{+}(d \geq 7)$ model with $\lambda \in(-\infty, 0)$, the replacement of $L_{ \pm}^{\text {top }}$ with $i\left|L_{ \pm}^{\text {top }}\right|$ transforms the "topological vacua positions" $\sigma_{j}^{2}=\left(\sigma_{j+2}^{2}\right)^{*}$ (with $\left.j=1,2,5,6\right)$ and the critical exponents $s_{\text {top }}^{p}$ and $s_{\text {top }}^{q}$ into complex numbers. Again as in the GB case (see App. B) the contribution $G_{t o p}^{Q T}(\sigma)$ of these "topological vacua" to the $\xi$ has the following real form:

$$
\begin{aligned}
G_{Q T}^{t o p} & =\prod_{j=1,5}^{2,6}\left[\left(\sigma^{2}-\left(\sigma_{j}^{2}\right)^{R}\right)^{2}+\left(\left(\sigma_{j}^{2}\right)^{I}\right)^{2}\right]^{-\delta_{j}^{R}} \exp \left(2 \delta_{j}^{I} \arctan \left(\frac{\left(\sigma_{j}^{2}\right)^{I}}{\sigma^{2}-\left(\sigma_{j}^{2}\right)^{R}}\right)\right) \\
\delta_{j}^{R} & =\frac{s_{j}^{R}}{\left(s_{j}^{R}\right)^{2}+\left(s_{j}^{I}\right)^{2}}, \quad \delta_{j}^{I}=\frac{s_{j}^{I}}{\left(s_{j}^{R}\right)^{2}+\left(s_{j}^{I}\right)^{2}}
\end{aligned}
$$

The separation of the real and imaginary parts of the $s_{t o p}^{q / p}$ and $\sigma_{j}^{2}$ in the case when both topological scales are imaginary can be realized as in the GB case, described in App. B.

- Massless and top-massless phases. The list of the $\mu_{ \pm}$models having one minimal topological scale $L_{+}^{\text {top }}$ and such that the both central functions $c(\sigma)$ and $a(\sigma)$ are positive within the intervals $\left(\sigma_{I R}, \sigma_{U V}\right)$ and $\left(\sigma_{U V}, \sigma_{\text {top }}^{+}\right)$, includes:

$$
\text { - } \mu_{+}(d \geq 7), \frac{d(d-4)}{3(d-2)^{2}}<\lambda<\frac{1}{3}, \quad \text { • } \mu_{-}(d \geq 9), \frac{(d-8)(d-4)}{3(d-6)^{2}}<\lambda<\frac{1}{3}
$$

Their holographic RG description is similar to the corresponding $d=5$ models.

\footnotetext{
${ }^{55}$ which are expected to be ghost free and stable w.r.t. small fluctuations of the metrics and of the matter field
} 
Although the explicit holographic form of the p.e.f requirements for $Q F T_{d-1}$ dual to $d \geq 7$ cubic QT Gravity is yet unknown, we expect that the massless RG flows for $\sigma \in\left(s_{U V}, s_{I R}\right)$ do represent unitary $Q F T_{d-1}$ 's, while the corresponding "top-massless phases" are physically inconsistent. This leads us to make a conjecture that only the holographic type (a) models can give rise to unitary and causal $Q F T_{d-1}$ models.

- $d \geq 7$ massless-to-massive phase transitions. As we have shown in Sect.5, for negative values of $\lambda$ and $\mu$, both central functions $a(l)$ and $c(l)$ are automatically positive for all the $d \geq 7$ QT gravity models. Therefore we have no topological vacua (i.e. both $L_{ \pm}^{t o p}$ are imaginary) and also no real zeros of the $a(\sigma)$ exist for all the values of $\sigma \in R$. Under these conditions the corresponding scale factor, representing a chain of two DW's $A d S(I R) / A d S(U V) / n . s$., gives rise to one massless phase $\left(\sigma_{I R}, \sigma_{U V}\right)$ and one standard massive phase $\left(\sigma_{U V}, \infty\right)$ quite similar to the GB case described in Sect.7.2. As usual they are characterized by $\xi\left(\sigma_{U V}\right)=\infty$ and $\xi\left(\sigma_{I R}\right)=0$ (the former one) and by the specific value $M_{Q T}=1 / \xi(\infty)$ of the mass gap (the later one), obtained by taking appropriate limits of the eqs. (7.16) and (7.21). Let us emphasize that the $Q F T_{d-1}$ 's dual to the $d \geq 7$ QT Gravity for negative $\lambda$ and $\mu$, exhibit standard (GB-like) massless-to-massive phase transition, which are absent in the corresponding $d=5$ models.

\subsubsection{Maximal scale massless phase}

An important new feature of the $Q F T_{d-1}$ 's (with $d \geq 7$ ) dual to the cubic Quasi-Topological Gravity is that for a specific range of values of the Lovelock's couplings $\lambda$ and $\mu$, namely

$$
\text { - } \mu_{+}(d \geq 7), \quad \frac{1}{4}<\lambda<\frac{1}{3}, \quad \bullet \mu_{-}(d \geq 7), \quad 0<\lambda<\frac{1}{3}
$$

the a/c-Theorem requirements can be satisfied in two distinct (and disconnected) regions: (1) of minimal fundamental scales $L_{+}^{a / t o p}$ such that $L_{U V}>L_{I R}>L_{+}^{a / t o p}$ (studied in Sect.7.4.1); (2) of maximal fundamental $L_{-}^{c^{\prime}}$ scale $^{56}$ such that $L_{I R}<L_{U V}<L_{-}^{c^{\prime}}<L_{-}^{\text {top }}<$ $L_{+}^{\text {top }}$.

In the case of the considered quartic Superpotential $W(\sigma)=-B\left[\left(\sigma^{2}-x_{0}\right)^{2}+D\right]$ with the $B, D$ and $x_{0}$ all positive, when the above new maximal scale restriction is imposed, we have no real solutions of the topological vacua equations (7.6), as one can easily verify from their explicit solutions (7.17). Therefore we can not realize the maximal scale requirements for positive signs of the parameters of superpotential. As it was suggested in ref. [5], certain flat DW solutions of the Quasi-Topological Gravity, satisfying the maximal scale conditions, can be constructed by keeping the quartic form of $W(\sigma)$ and the condition $W<0$, but considering the case of negative $B$ and $D$ and $x_{0}>0$, i.e. by taking $\tilde{W}(\sigma)=$ $|B|\left[\left(\sigma^{2}-x_{0}\right)^{2}-|D|\right]<0$. This Superpotential represents the standard Higgs potential, that satisfies the $\tilde{W}<0$ condition for the values of $\sigma$ restricted to the interval $\sigma^{2} \leq x_{0}+\sqrt{|D|}$ with $x_{0} \leq \sqrt{|D|}$. The solutions for the scale factor of such maximal scale DWs and related to them correlations lengths $\xi(\sigma)$ are again given by eqs. (7.16), (7.17) and (7.18), but with $B$ and $D$ replaced by $-|B|$ and $-|D|$. As a consequence the critical points $\tilde{\sigma}_{I R}^{2}=x_{0}$, i.e. the minima of the new $\tilde{W}$ are now of IR type, while its maximum $\sigma_{U V}=0$ is becoming

\footnotetext{
${ }^{56}$ or $L_{t o p / h}^{-}$in case the requirements of the Modified a/c-Theorem are imposed
} 


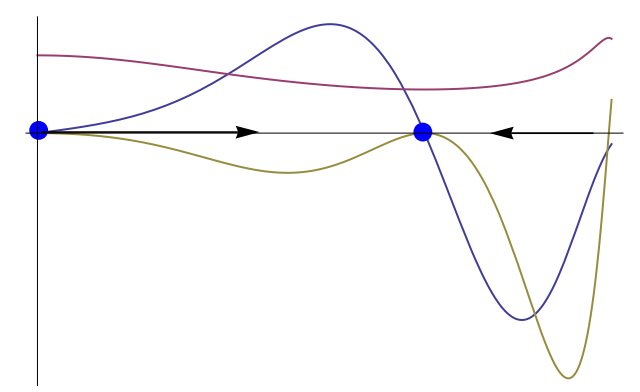

Figure 11. RG flows in the maximal scale region: the $-\beta(\sigma)$-function is the blue curve; the $c(\sigma)$-function is the red one and the $\frac{d c(\sigma)}{d l}$-function is drawn in beige; the arrows indicate the directions of the massless RG flow.

the new UV -critical point, since $\tilde{s}_{U V}(-|B|,-|D|)=s_{I R}(B, D)$ is positive. The topological $\sigma_{\text {top }}^{-}$vacua is also of UV type.

The above analysis of the critical points of the dual QFT (respecting the maximal scale restrictions), completed by the behaviour of $\xi(\sigma)$, namely that $\xi(0)=\infty, \xi\left( \pm \sqrt{x_{0}}\right)=0$ and $\xi\left( \pm \sigma_{\text {top }}^{-}\right)=\infty$, lead to the following conclusion: the phase structure of such maximal scale dual $Q F T_{d-1}$ 's consists of two identical massless phases $\left(-\sqrt{x_{0}}, 0\right)$ and $\left(0, \sqrt{x_{0}}\right)$. The plots of the corresponding $\beta(\sigma)-, c(\sigma)$ - and $d c / d l$-functions, shown on Fig.(11), confirm the existence of such maximal scale massless RG flows, satisfying all the conditions of the $a / c$ Theorem for certain higher dimensional QFT's dual of the $d \geq 7$ QT Gravity models.

It is important to mention that contrary to the case of the minimal scale models that are characterized by $C_{\Phi \Phi \Phi}^{U V} \neq 0$, all the maximal scale models based on the above Higss-like superpotential have vanishing $U V$-structure constant (and non-zero IR-ones) which makes unclear their identification as certain perturbed $C F T_{d-1}$ models. This problem is however absent in the case of more complicated maximal scale QFT models dual to QT Gravity with an appropriately modified superpotential obtained from $\tilde{W}$ by adding to it a cubic term.

\section{Conclusions}

8.1. Motivations and goals. The Holographic RG group methods [2, 3] are known to have many important applications in the investigation of the RG flows and the phase structure in the strong coupling limits of certain $S U\left(N_{c}\right) \mathcal{N}=4$ supersymmetric $C F T_{d-1}$ 's with an appropriate massive term for the chiral scalar super-fields added. The main tool in such off-critical version of the $A d S_{5} / C F T_{4}$ correspondence are the DW's solutions of the corresponding dual five-dimensional gauged $\mathcal{N}=8$ Einstein super-gravity. One of the main characteristics of the $\mathcal{N}=4$ susy $C F T_{4}$ 's is that they must have equal central charges $c=a$. Since the less supersymmetric $\mathcal{N}=0,1,2 C F T_{4}$ 's by definition have distinct central charges $c \neq a$, their holographic critical and off-critical description are known to require certain "higher derivatives" extensions of the Einstein (super)Gravity, as for example the GB and 
the cubic or quartic Quasi-topological Gravities [17, 18, 64]. Again for the construction of the corresponding "holographic" $\beta(\sigma)$ - function the explicit forms of the DW's of such extended Gravities are needed. The problem we have addressed and solved in the present paper concerns the holographic description of the massless and the massive phases and of the phase transitions occurring in a family of $c \neq a$ (zero temperature) $Q F T_{d-1}$ 's, by using the recently constructed GB and cubic QT Gravities DW's [5]. As we have already mentioned, the main advantage of the superpotential method $[5,6,20,37]$ is that it allows to easily implement all the unitarity and positive energy fluxes conditions on the basic UVand IR- $C F T$ data $\left(L_{k}: s_{k}, c_{k}, a_{k}, t_{4}^{k}\right)$ in order to derive a set of consistency conditions on the matter superpotential parameters and on the gravitational couplings $\lambda$ and $\mu$. The RG evolution of this conformal data, giving rise to the massless and massive RG flows in the corresponding dual unitary $Q F T_{d-1}$ 's, is described by specific chains of DW's.

Let us emphasize once more that the purpose of the investigations presented in the this paper is to realize a comparative test of the way the off-critical properties of the dual $c \neq a$ holographic $Q F T_{d-1}$ 's depend on the particular choice of the extended "higher curvature" Gravities and on the specific form of the scalar matter superpotential as well. Our attention is always concentrated on the question of whether the specific requirements of the unitarity and causality consistency of corresponding massive and massless phases can be satisfied within the frameworks of the considered examples of extended EH gravity-matter models. The motivations to consider the particular case of Holographic RG flows in the QFT's dual to the GB and the cubic Quasi-Topological Gravity [17] are:

- their remarkable feature to lead to second order equations for both the flat DWs and for the corresponding linear fluctuations around such DW's background;

- they allow to reproduce the most general $a \neq c$ and $t_{4} \neq 0$ non-supersymmetric CFT's $[7,8,11]$;

- for $d=5$ their dual $Q F T_{4}$ 's turns out to have quite a reasonable off-critical phase structure and describe phase transitions expected to take place in certain approximations of the $Q C D_{4}$, as well as in the description of some features of the quark-gluon plasma hydrodynamics [7, 8, 24, 26];

- they are expected to have an application to the consistent holographic definition and also for the derivation of some important off-critical characteristics of the entanglement entropy in the dual "boundary" non-conformal $Q F T_{d-1}$ 's (in different geometries and b.c.'s), as well as for its calculation in such DW's backgrounds [62, 65, 73].

8.2. Summary of the main results. Starting from the explicit constructions of the GB and QTG domain walls and the well known critical unitarity and energy fluxes positivity conditions, we have addressed the following two problems:

$\mathcal{P} 1$ : to establish the off-critical consistency requirements that the corresponding $a(l)$, $c(l)$ and $\beta(l)$-functions have to satisfy;

$\mathcal{P} 2$ : to describe the phase structure, the holographic RG flows and the nature of the phase transitions in the dual non-conformal $Q F T_{d-1}$ 's, which are compatible with the corresponding $a / c$-Theorem restrictions. 
- Holographic a/c-Theorems. Taking as a basic ingredient the unitarity and p.e.f. consistency of the $C F T_{d-1}(U V / I R)$-data, we have extended them to the massless phases of the conjectured dual $Q F T_{d-1}$ 's, giving rise to the holographic versions $[3,7,9]$ of the "a/c-Theorems" of Zamolodchikov and Cardy $[12,13]$. The main questions to be answered are: (1) about the behaviour and of the eventual monotonic properties of the $c(l)$-central function and (2) about the conditions the parameters of the considered models should satisfy in order to have both central functions always positive and respecting the p.e.f. requirements. Our proofs have established the specific minimal or/and maximal scales restrictions on the UV and IR "physical scales" $L_{U V / I R}$, which guarantee the validity of the different forms of the $a / c$-Theorem. Two qualitatively different behaviours have to be distinguished:

Minimal scale: All the QFT's models duals to the $d=5$ QT Gravity and to the $d \geq 5$ GB Gravity exhibit massless RG flows with both $a$ and c-central functions positive and monotonically decreasing, when the following minimal scale requirements $L_{U V}>L_{I R}>$ $L_{\min }$ are fulfilled. The specific exact (model dependent) values of these $L_{\min }$ are derived in Sect.6 from the conditions of positivity of the energy fluxes. For example, in all the $Q F T_{d-1}$ 's duals to the GB models of positive $\lambda$ we get $L_{\min }^{2}=2 L^{2} \lambda\left(d^{2}-5 d+10\right) /(d-$ $3)(d-4)$.

Maximal scale: All the QFT's duals to $d \geq 7$ QTG models admit two distinct type of massless RG flows: together with the above minimal scale version of the $a / c$-Theorem we have a new one with specific maximal scale restrictions $L_{I R}<L_{U V}<L_{\max }$, ensuring that again $c(l)$ is decreasing. However now we can also realize two quite different behaviours of the $c(l)$-function, namely: monotonically increasing $d c / d l>0$ in the case when $L_{\min }^{*}<$ $L_{I R}<L_{U V}<L_{\max }^{*}$ or non-monotonic $c(l)$ for $L_{I R}<L_{\max }<L_{U V}$. The values of the corresponding maximal/minimal scales $L_{\min }^{*}$ and $L_{\max }^{*}$ are derived in sect.5 and App. A.

The realization of all the important off-critical QFT's data [7-9] as functions of the running coupling $\sigma(l)$ and more precisely in terms of the superpotential $W(\sigma)$, allows us to derive the extensions of the $a$ and $c$-Theorems including the energy fluxes parameters $t_{2}(\sigma)$ and $t_{4}(\sigma)$ as well. Eventual applications of these results to the non-conformal $s Q G P$ hydrodynamics for the calculation of the bulk viscosity $\zeta$ and also for deriving formulas $(\eta / s)_{U V} \geq(\eta / s)_{I R}$ relating the UV and IR values of the the ratio of shear viscosity to entropy density, that takes place in the massless phase of the $Q F T_{4}$ dual to the GB model of positive $\lambda$, are discussed in Sect. 6 .

- Holographic RG flows and QFT's phase transitions. Given the explicit form (2.8) of the holographic $\beta_{W}(\sigma)$-function for our particular choice of quartic inverted Higgs superpotential $W(\sigma)$. The solutions of the corresponding Wilson RG equations (7.1) are known to provide all the information necessary for the description of the main characteristics of the QFT's phases and the nature of the phase transitions [33]. The knowledge of the analytic forms of the running coupling constant $\sigma(l)$, of the correlation length $\xi(\sigma)$ and of the reduced free energy $F(\sigma)$ for all the values of the coupling $\sigma$, allows us to identify the distinct intervals $\left(\sigma_{k}, \sigma_{k+1}\right) \in R$ between the critical points $\sigma_{k}$ 's as representing massive or massless phases. The detailed analysis of the off-critical behaviour of the considered models, performed in sect.7, leads us to the following conclusions about the "holographic 
phase structure" of the corresponding dual unitary $Q F T_{d-1}$ :

(1) QFT's duals to GB for negative $\lambda$. Massless unitary RG flow in $\left(\sigma_{I R}, \sigma_{U V}\right)$ and Massive phase $\left(\sigma_{U V}, \infty\right)$ with mass gap given by eq. (7.12); second order phase transition.

(2) QFT's duals to GB for positive $\lambda$. One massless phase $\left(\sigma_{I R}, \sigma_{U V}\right)$ and a new kind of a-massive phase $\left(\sigma_{U V}, \sigma_{a}^{c r}\right)$ with $a\left(\sigma_{a}^{c r}\right)=0$, the physical consistency of which is under question.

(3) $Q F T_{4}$ duals to $d=5 Q T G$. Only one well defined massless phase $\left(\sigma_{I R}, \sigma_{U V}\right)$. For the $\mu_{+}(d=5)$ model with $\lambda \in\left(\frac{5}{27}, \frac{1}{3}\right)$ and for the $\mu_{-}(d=5)$ model with $\lambda \in\left(-\infty, \frac{1}{3}\right)$ we have one massless and one a-massive phase as in the GB case (2). For the $\mu_{+}(d=5)$ model with $\lambda \in\left(-\infty, \frac{5}{27}\right)$ together with the standard massless RG flow within $\left(\sigma_{I R}, \sigma_{U V}\right)$, we can have a new "top-massless" phase $\left(\sigma_{U V}, \sigma_{\text {top }}^{+}\right)$, which however is violating the p.e.f. requirements.

(4) $Q F T_{d-1}$ duals to $d \geq 7 Q T G$-minimal scale. For $\lambda$ and $\mu$ both negative the massive-to-massless phase transition is as in GB case (1); the class of models listed in (7.20) has the same phase structure as in the GB case (2) above; the phase structure of the models (7.22) includes the physically inconsistent "top-massless" phase as in the $d=5$ case (3) above.

(5) $Q F T_{d-1}$ duals to $d \geq 7$ QTG -maximal scale. The $\mu_{+}$models with $\frac{1}{4}<\lambda<\frac{1}{3}$ and the $\mu_{-}$ones with $0<\lambda<\frac{1}{3}$ exhibit only one consistent massless phase, satisfying the $a / c$ Theorem and its modifications. They are described in Sects.5. and realized in Sect.7.4.

(6) Infinite order phase transitions. We can have also two marginal degeneration limits $L_{U V}=L_{I R}$ and $L_{U V}=L_{t o p}$ (in the case when we have at least have one topological vacuum) that lead to essential singularities in the free energy as in eq. (7.15). They are known to describe infinite order BKT-type phase transitions (or Miransky scaling [67$69,71]$ ), that are important in the description of $Q C D_{4}$ of large number of flavors $N_{f}>>1$ within the conformal window $4 \leq N_{f} / N_{c}<11 / 2^{57}$.

8.3. Open problems. We have to mention that our investigation of the critical phenomena in certain unitary QFT's duals to the GB and QT gravity-mater models left unanswered few important questions. The description of the different massive and massless phases by using of RG methods, based on the analytic properties and the asymptotic behaviour of the corresponding reduced free energy, needs to be completed by further analysis of the following three problems:

- the stability and the bulk causality of the specially selected chains of DWs, describing the phase structure of the considered dual QFT's;

- the holographic calculation of the off-critical mass-spectrum and of the 2-point correlations functions of the relevant operator $\Phi_{\sigma}\left(x_{i}\right)$, driving the RG flows;

- the off-critical extension of the CFT's conditions for the positivity of the energy fluxes.

\footnotetext{
${ }^{57}$ the $Q F T_{4}$ 's duals to the marginally degenerated $d=5$ GB DW's are expected to represent $\mathcal{N}=1$ susy $S U\left(N_{c}\right) Q C D_{4}$ with $N_{f}$ flavors within the corresponding "superconformal window" $3 / 2 \leq N_{f} / N_{c} \leq 3$ [70]
} 
The solution of the first two problems requires further studies of the linear fluctuations (of both the metrics and of the matter field $\sigma$ ) around the special GB and cubic QTG DW's backgrounds, we have used in the description of the off-critical behaviour of the corresponding dual QFT's. Although the physical vacua are by construction perturbatively stable $[7,9,10,17]$, the knowledge of the spectrum of the small fluctuations around such DWs is further needed in order to establish their proper stability, as well as for revealing the eventual causality violations that might occur in the bulk, as in the examples studied in refs. $[7,10,16]$. An important fact to be mentioned here is that the corresponding DW's fluctuation equations ${ }^{58}$ obtained from the cubic Quasi-Topological gravity-matter action (2.1) are known to be second order differential equations [9, 17], rather similar to the ones of the EH gravity-matter case.

The third problem concerns further off-critical restrictions that eventually have to be imposed on the DW's and on the gravitational couplings $\lambda$ and $\mu$ in order to ensure the unitarity of the dual QFT's. Sect.6.4 contains a preliminary discussion of how one can define and calculate the energy fluxes in the perturbed $C F T$ 's, representing the considered non-conformal QFT's, by applying the well known conformal perturbations methods, quite similar to the ones used in ref. [44] for the calculation of the free energy.

8.4. Few problems for further research. The results we have obtained by using specially selected DW's of the GB and QT Gravities with the holographic $a / c$-Theorems and the energy fluxes positivity conditions properly implemented, provide examples of dual unitary QFT's having quite reasonable massless phases. However the only $Q F T_{4}$ 's that represent unitary (and eventually stable) massive phase are those ones duals to the GB models of negative $\lambda$ with $t_{4}=0$. They are known to correspond to $\mathcal{N}=1$ supersymmetric QFT's. Therefore the problem of the holographic description of non-supersymmetric $c \neq a Q F T_{4}$ 's with $t_{4} \neq 0$, that have well defined massive phase still remains open. Leaving aside the possible relation of such models with certain large $N_{c}$ limits of the $Q C D_{4}$, we shall mention here few generalizations of the models and of the methods used in the present paper that might lead to more realistic $t_{4} \neq 0$ massive $Q F T_{4}$ :

- to take as a starting point different "higher derivatives" $d=5$ Gravities of Lovelock type, as for example the recently constructed quartic Quasi-Topological gravity [64], the extended Born-Infeld (BI) models [74] or certain extensions of the bi-metric massive gravity [75]. In all these cases both the superpotential method and the proofs of the corresponding $a / c$-Theorems are expected to have rather straightforward generalizations;

- to keep unchanged the gravitational cubic QTG part of the action (2.1), but to consider other more realistic forms of the matter superpotential. One can take as examples superpotentials, involving many scalar fields of sigma model type, or else certain well known super-gravity induced superpotentials, like in the case of EH super-gravity models [3]. Again an important technical problem to be resolved is the construction of the corresponding DW's by an appropriate extension of the superpotential method, that also allows

\footnotetext{
${ }^{58}$ differently from the case of the fluctuations equations around the cubic QTG black-hole solutions that are of fourth order [17].
} 
to derive the holographic $\beta$-function and the form of the central functions [76].

It is worthwhile to also mention another promising direction of related research, concerning the investigation of the marginally degenerated DW's in the GB and QTG models of at least three physical vacua [5] and their applications to the holographic description of certain infinite order BKT (or Miransky ) phase transitions in the dual QFT's. Such behaviour is expected to take place in the $Q C D_{4}$ in the Veneziano limit [72] of large number of flavors $N_{f}$ and colors $N_{c}$, but of finite ratio within the conformal (non-supersymmetric) window $x_{c} \leq N_{f} / N_{c} \leq 11 / 2[67-69,71]$ with $x_{c} \approx 4$.

Acknowledgements. We are grateful to A.L.A.Lima for critical reading of the manuscript and for his suggestions for improvements. 


\section{Appendices}

\section{A The proof of $d \geq 7 a / c$ - Theorems}

\section{A.1 $\mu_{+}$model}

The Standard $\mu_{+}$model $a / c$-Theorem valid for $d \geq 7$ reads:

The conditions that $a(l)$ - and $c(l)$-central functions are both positive and monotonically decreasing during the $R G$ flow between two consecutive critical points $\sigma_{U V}$ and $\sigma_{I R}$ are given by:

$$
\begin{array}{ll}
(1) \bullet & -\infty<\lambda<\frac{1}{4}: \quad f_{I R}<f_{+}^{a}\left(\lambda ; \mu_{+}\right), \\
(2) \bullet & \frac{1}{4}<\lambda<\frac{d(d-4)}{3(d-2)^{2}}: \quad f_{I R}<f_{+}^{a}\left(\lambda ; \mu_{+}\right) \text {or } f_{U V}>f_{-}^{c^{\prime}}\left(\lambda ; \mu_{+}\right), \\
(3) \bullet & \frac{d(d-4)}{3(d-2)^{2}}<\lambda<\frac{1}{3}: \quad f_{I R}<f_{+}^{t o p}\left(\lambda ; \mu_{+}\right) \text {or } f_{U V}>f_{-}^{c^{\prime}}\left(\lambda ; \mu_{+}\right) .
\end{array}
$$

Proof. Similarly to the $d=5$ case, the sign of $\mu_{+}$is crucial in the investigation of the intervals of $\lambda$ where $f_{ \pm}^{\eta}$ are real positive or complex. Taking into account their explicit forms (3.2) we realize that:

$$
-\infty<\lambda<\frac{1}{4}, \quad \mu_{+}(\lambda) \geq 0, \quad f_{+}^{\eta}(\lambda)>0, \quad f_{-}^{\eta}(\lambda)<0, \quad \eta=t o p, a, c^{\prime}
$$

and as a consequence the following ordering (see also Fig.(12(a))):

$$
0<f_{+}^{a}<f_{+}^{t o p}<f_{+}^{c^{\prime}},
$$

takes place. For positive values of $\mu$ the requirements:

$$
\left(f+\left|f_{-}^{\eta}\right|\right)\left(f-f_{+}^{\eta}\right)<0, \quad \eta=\text { top }, a, c^{\prime} \quad \text { or } \quad 0<f<f_{+}^{\eta}, \quad \text { i.e. } \quad 0<f<f_{+}^{a}
$$

guarantee that the conditions of the Standard a/c-Theorem: $a>0, c>0$ and $d c / d l<0$ are fulfilled, which proves the case (1) of the above Theorem.

As we have demonstrated in Sect.2.1., in the remaining two cases (a2) and (a3) where $\mu_{+}$is negative and both $f_{ \pm}^{\text {top }}>0$ are positive we have the following restrictions ensuring $c(f)>0$ :

$$
\begin{array}{llll}
\text { - } & \frac{1}{4}<\lambda<\frac{8}{27}: & h<0, \quad c>0 \quad \text { for } \quad 0<f<f_{+}^{t o p} \quad \text { and } \quad f>f^{h}>f_{-}^{t o p}, \\
\text { - } \frac{8}{27}<\lambda<\frac{1}{3}: \quad h \geq 0, \quad c>0 \quad \text { for } \quad 0<f<f_{+}^{t o p} \quad \text { and } \quad f>f_{-}^{t o p}
\end{array}
$$

In order to satisfy all the requirements $\left(f-f_{-}^{\eta}\right)\left(f-f_{+}^{\eta}\right) \geq 0$ of the a/c-Theorem, we next observe that according to the arguments presented in Sect.3.2. above, both $f_{ \pm}^{c^{\prime}}>0$ are positive within the interval $\lambda \in(1 / 4,1 / 3)$. Therefore the condition $d c / d l<0$ is satisfied for all the $f$ 's such that:

$$
\text { - } 0<f<f_{+}^{c^{\prime}} \quad \text { and } \bullet f>f_{-}^{c^{\prime}},
$$


Given the explicit form (3.2) of $f_{ \pm}^{a}\left(\lambda, \mu_{+}\right)$, the description of their properties and of the corresponding conditions for positivity of $a(\sigma)$-central function requires a bit more involved case-by-case analysis. It is relatively easy to demonstrate that the solutions of the reality condition:

$$
\frac{9 \lambda^{2}(d-6)(d-2)}{(d-4)^{2}}+2-9 \lambda \geq-2(1-3 \lambda)^{3 / 2}
$$

are given by:

- $0<f_{+}^{a}<f_{-}^{a} \quad$ for $\quad \lambda \in\left(\frac{1}{4}, \lambda_{a}^{+}\right), \quad \bullet \quad f_{ \pm}^{a} \quad$ complex for $\quad \lambda \in\left(\lambda_{a}^{+}, \frac{1}{3}\right), \quad \lambda_{a}^{+}=\frac{d(d-4)}{3(d-2)^{2}}$

As we have mentioned in Sect.2.1. in the region $\lambda \in(1 / 4,8 / 27)$, where $h<0$, we have to introduce a new maximal scale $f^{h}=-\lambda / 2 \mu_{+}+\sqrt{\left(\lambda^{2}+4 \mu_{+}\right) / 4 \mu_{+}^{2}}>f_{-}^{t o p}$, instead of $f_{-}^{t o p}$. Therefore the description of the conditions of validity of a/c-Theorem for $\mu_{+}$-model, depends on whether $\lambda_{+}^{a}<8 / 27$ or $\lambda_{+}^{a} \geq 8 / 27$. In the proof $d=7 \mathrm{a} / \mathrm{c}$-Theorem, due to the fact that $\lambda_{+}^{a}=7 / 25<8 / 27$, we have to distinguish the following three cases:

$$
\begin{aligned}
& \text { - } \frac{1}{4}<\lambda<\frac{7}{25}: \quad 0<f_{+}^{a}<f_{+}^{t o p}<f_{+}^{c^{\prime}}<f_{-}^{a}<f_{-}^{t o p}<f^{h}<f_{-}^{c^{\prime}} \quad \text { or } \\
& 0<f_{+}^{a}<f_{+}^{t o p}<f_{-}^{a}<f_{+}^{c^{\prime}}<f_{-}^{t o p}<f^{h}<f_{-}^{c^{\prime}} ; \\
& \bullet \frac{7}{25}<\lambda<\frac{8}{27}: \quad 0<f_{+}^{\text {top }}<f_{+}^{c^{\prime}}<f_{-}^{\text {top }}<f^{h}<f_{-}^{c^{\prime}}, \\
& \text { - } \frac{8}{27}<\lambda<\frac{1}{3}: \quad 0<f_{+}^{t o p}<f_{+}^{c^{\prime}}<f_{-}^{t o p}<f_{-}^{c^{\prime}} .
\end{aligned}
$$

The particular ordering of all the $f_{ \pm}^{\eta}(\lambda, d=7)$ with $\eta=a, c^{\prime}, h$, top is established graphically as plotted on Fig.(12(b)). Since $\lambda_{+}^{a}=8 / 27$ for $d=8$, we have to consider separately only the following two regions : (1) $\frac{1}{4}<\lambda<\frac{8}{27}$ with the same properties of all the $f^{\eta \prime}$ 's as in the first region in (A.10) above, and (2) $\frac{8}{27}<\lambda<\frac{1}{3}$ literally repeating the third region of $d=7$ case. Finally, for $d \geq 9$ we always have that $\frac{8}{27}<\lambda_{+}^{a}<\frac{1}{3}$. Therefore, similarly to the $d=7$ case, the following three cases should be analysed separately:

$$
\begin{aligned}
& \text { - } \frac{1}{4}<\lambda<\frac{8}{27}: \quad 0<f_{+}^{a}<f_{+}^{\text {top }}<f_{+}^{c^{\prime}}<f_{-}^{a}<f_{-}^{\text {top }}<f^{h}<f_{-}^{c^{\prime}} \quad \text { or } \\
& 0<f_{+}^{a}<f_{+}^{t o p}<f_{-}^{a}<f_{+}^{c^{\prime}}<f_{-}^{t o p}<f^{h}<f_{-}^{c^{\prime}} ; \\
& \text { - } \frac{8}{27}<\lambda<\frac{d(d-4)}{3(d-2)^{2}}: \quad 0<f_{+}^{a}<f_{+}^{t o p}<f_{+}^{c^{\prime}}<f_{-}^{a}<f_{-}^{\text {top }}<f_{-}^{c^{\prime}} \quad \text { or } \\
& 0<f_{+}^{a}<f_{+}^{t o p}<f_{-}^{a}<f_{+}^{c^{\prime}}<f_{-}^{t o p}<f_{-}^{c^{\prime}} ; \\
& \text { - } \frac{d(d-4)}{3(d-2)^{2}}<\lambda<\frac{1}{3}: \quad 0<f_{+}^{\text {top }}<f_{+}^{c^{\prime}}<f_{-}^{\text {top }}<f_{-}^{c^{\prime}} \text {. }
\end{aligned}
$$

Notice that (a) the only difference between the first two cases in (A.11) is in the presence of the new intermediate scale $f^{h}$ when the interval $1 / 4<\lambda<8 / 27$ is considered, due to the fact that $h<0$ is negative there; (b) the properties of the $f^{\eta}$ 's that are positive in the last region are common for all the $d \geq 7$. It is important to mention that the two different 
orderings $f_{+}^{c^{\prime}}<f_{-}^{a}$ and $f_{-}^{a}<f_{+}^{c^{\prime}}$ present in all the cases when $\lambda \in\left(1 / 4, \lambda_{+}^{a}\right)$ reflect the fact that it exists one critical value $\lambda_{c r}^{a c^{\prime}}$, where the two scales $L_{+}^{c^{\prime}}\left(\lambda_{c r}\right)=L_{-}^{a}\left(\lambda_{c r}\right)$ do coincide. As one can see from fig.(12(b)) it is exactly the point when the curves $f_{+}^{c^{\prime}}(\lambda)$ and $f_{-}^{a}(\lambda)$ cross each other.

It is now straightforward to conclude that the conditions : $\left(f-f_{-}^{\eta}\right)\left(f-f_{+}^{\eta}\right) \geq 0$ for validity of the Standard $a / c$-Theorem for the $\mu_{+}$-model in the region $\lambda \in(1 / 4,1 / 3)$ are given by :

(1a) $0<f_{v a c}<f_{+}^{a}\left(\lambda ; \mu_{+}\right)$and (1b) $f>f_{-}^{c^{\prime}}\left(\lambda ; \mu_{+}\right)$for $\lambda \in\left(\frac{1}{4}, \frac{d(d-4)}{3(d-2)^{2}}\right)$ due to the fact that $f_{+}^{a}$ is defining the minimal scale and $f_{-}^{c^{\prime}}$ - the maximal one in this region, while for $\lambda \in\left(\frac{d(d-4)}{3(d-2)^{2}}, 1 / 3\right)$ we have:

(2a) $0<f<f_{+}^{\text {top }}\left(\lambda ; \mu_{+}\right)$of different minimal scale and (2b) $f>f_{-}^{c^{\prime}}\left(\lambda ; \mu_{+}\right)$, i.e. with the same maximal scale.

Notice that the of presence of the new intermediate scale defined by $f^{h}$ in the regions of negative $h<0$ that leads to certain differences between the cases $d=7, d=8$ and $d \geq 9$ as described by eqs. (A.10) and (A.11) above is not changing the requirements of the Standard Theorem. They have in fact the same form for all the models with $d \geq 7$.

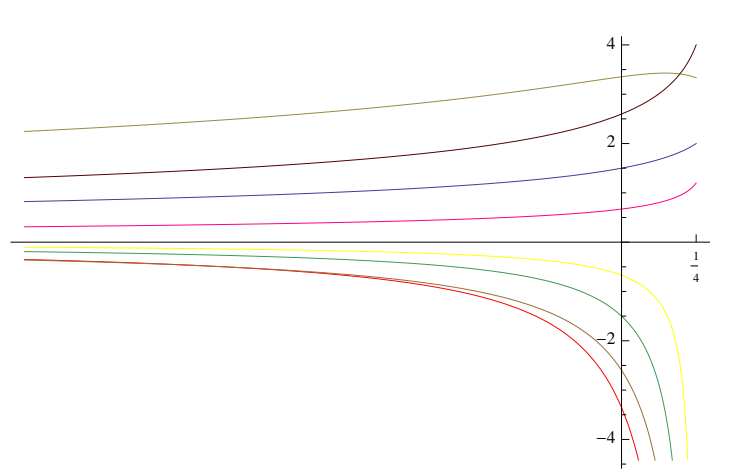

(a)

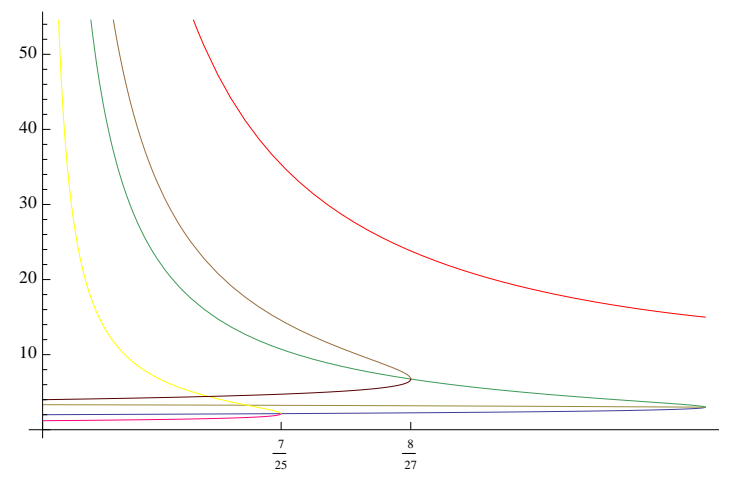

(b)

Figure 12. The $f_{ \pm}^{\eta}\left(\lambda ; \mu_{+}\right)$curves in $d=7 \mu_{+}$model: $f_{+}^{\text {top }}$ is depicted in blue; $f_{+}^{a}$ in pink; $f_{+}^{c^{\prime}}$ in beige; $f_{+}^{h}$ in dark brown; $f_{-}^{t o p}$ in green; $f_{-}^{a}$ in yellow; $f_{-}^{c^{\prime}}$ in red and $f_{-}^{h}$ in bright brown. Fig.(a) represents the $\lambda<\frac{1}{4}$ interval and Fig.(b) the $\frac{1}{4}<\lambda<\frac{1}{3}$ one; both with reference point $(1 / 4,0)$. The special point $\lambda=\frac{7}{25}$ marks the end of the curves $f_{ \pm}^{a}$.

\section{A.2 $\mu_{-}$model}

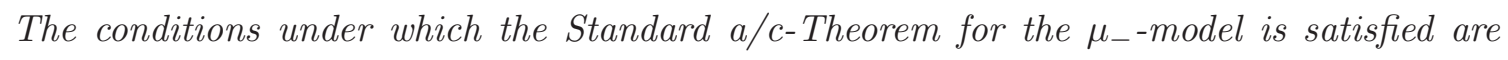
given by:

- $d=7,8: \quad 0<\lambda<\frac{1}{3}: \quad f<f_{+}^{t o p}\left(\lambda ; \mu_{-}\right) \quad$ or $\quad f>f_{-}^{c^{\prime}}\left(\lambda ; \mu_{-}\right)$

- $d \geq 9: \quad 0<\lambda<\frac{(d-8)(d-4)}{3(d-6)^{2}}: \quad f<f_{+}^{a}\left(\lambda ; \mu_{-}\right) \quad$ or $\quad f>f_{-}^{c^{\prime}}\left(\lambda, \mu_{-}\right)$

- $d \geq 9: \quad \frac{(d-8)(d-4)}{3(d-6)^{2}}<\lambda<1 / 3: \quad f<f_{+}^{t o p}\left(\lambda ; \mu_{-}\right) \quad$ or $\quad f>f_{-}^{c^{\prime}}\left(\lambda ; \mu_{-} \gamma\right.$ 
where $f$ is denoting $f_{I R}$ or $f_{U V}$ in the case of minimal/maximal scale correspondingly.

Proof. Since both $f_{ \pm}^{\eta}>0$ (for $\eta=c^{\prime}$, top) are known to be positive for $\lambda \in(0,1 / 3]$, it remains the analysis of the properties of $f_{ \pm}^{a}$ 's. Taking into account their form (3.2), similarly to the the $\mu_{+}$-model case (see eq. (A.9) is straightforward to realize that the corresponding reality condition:

$$
\frac{9 \lambda^{2}(d-6)(d-2)}{(d-4)^{2}}+2-9 \lambda \geq 2(1-3 \lambda)^{3 / 2},
$$

is satisfied only for $\lambda \leq \lambda_{-}^{a}=\frac{(d-8)(d-4)}{3(d-6)^{2}}$. Within the interval $\lambda \in\left(\lambda_{-}^{a}, 1 / 3\right]$ the $f_{ \pm}^{a}$ 's are complex. In the case $d=7$ we have $\lambda_{-}^{a}=-1$ and therefore $f_{ \pm}^{a}$ are indeed complex in the interval $(0,1 / 3)$ we are investigating. Similarly, for $d=8$ the $\lambda_{-}^{a}(d=8)=0$ is vanishing and again we have complex $f_{ \pm}^{a}$ 's. The ordering of the remaining real and positive $f_{\eta}$ 's is plotted on Fig.(13(a)) (for $d=7$ ) and without lost of generality for $d=7,8$ it is given by:

$$
0<f_{+}^{t o p}<f_{+}^{c^{\prime}}<f_{-}^{t o p}<f_{-}^{c^{\prime}}
$$

Remembering that the conditions $\left(f-f_{-}^{\eta}\right)\left(f-f_{+}^{\eta}\right) \geq 0$ for validity of the standard Theorem are identical to the ones of the $\mu_{+}$model for negative $\mu_{+}<0$, we derive the restrictions on $f_{U V / I R}$ for $d=7,8$ as stated by eq. (A.12) in the beginning of this subsection.

Due to the fact that for $d \geq 9$ the corresponding values of $\lambda_{-}^{a}<1 / 3$ are always positive, we now have to consider separately the case $\lambda \in\left(0, \lambda_{-}^{a}\right)$, where both $f_{ \pm}^{a}\left(\lambda, \mu_{-}\right)>0$ are positive, from the remaining part of the interval,i.e. $\lambda \in\left(\lambda_{-}^{a}, 1 / 3\right)$ where $f_{ \pm}^{a}\left(\lambda, \mu_{-}\right)$still remain complex. The corresponding (real and positive) $f^{\eta}(\lambda)$ curves for $d=9$ are shown on Fig.(13(b)). Hence, quite similarly to the $\mu_{+}$-model, we can conclude that the following orderings

- $\lambda \in\left(0, \lambda_{a}^{-}\right) \quad 0<f_{+}^{a}<f_{+}^{t o p}<f_{+}^{c^{\prime}}<f_{-}^{a}<f_{-}^{t o p}<f_{-}^{c^{\prime}}$

- $\lambda \in\left(\lambda_{a}^{-}, \frac{1}{3}\right) \quad 0<f_{+}^{\text {top }}<f_{+}^{c^{\prime}}<f_{-}^{\text {top }}<f_{-}^{c^{\prime}}$

take place. It is then evident that the conditions of validity of the Standard Theorem (same as above) are indeed the ones given by eqs. (A.13) and (A.14).

\section{B GB domain walls for negative $\lambda$}

The scale factor $e^{2 A(\sigma)}$ of GB DW's for the considered Higgs-like superpotential $W$ can be easily obtained by integrating the RG equation (2.8), which gives rise to eq. (7.7) representing its general solution. In the case of negative values of $\lambda$, due to the absence of topological vacua, the topological scale $L_{\text {top }}=L \sqrt{2 \lambda}$ that is used in the parametrisation of the corresponding "topological vacua" positions $\sigma_{j}^{2}$ and of the "critical exponents" $s_{t o p}^{j}$ has to be replaced by $i\left|L_{t o p}\right|=i L \sqrt{2|\lambda|}$. Although both the $s_{t o p}^{j}$ and $\sigma_{j}^{2}$ become complex numbers, we are going to show that the scale factor (7.7) remains indeed a well defined real function of $\sigma$. We next assume that the $\lambda>0$ vacua stability requirement $L_{U V}>L_{\text {top }}$, i.e. $x=L_{t o p} / L_{U V}<1$, take place in the considered $\lambda<0$ case as well, in order to keep the 


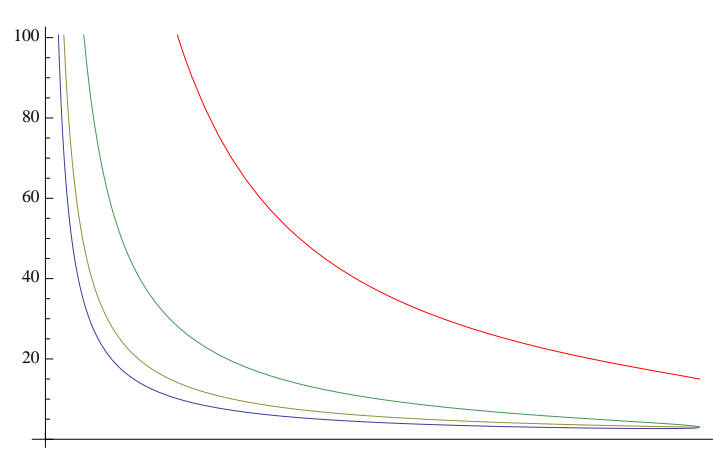

(a)

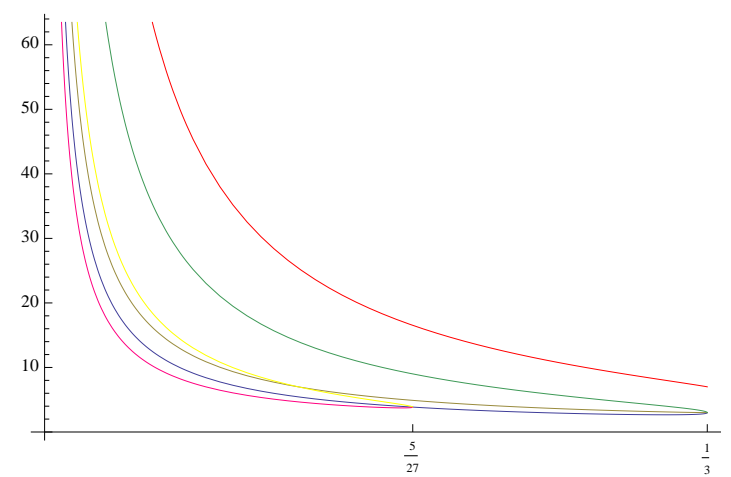

(b)

Figure 13. The $f_{ \pm}^{\eta}\left(l ; \mu_{-}\right)$curves in $d=7$ for $0<l<\frac{1}{3}$ are represented on Fig.(a): the curve $f_{+}^{\text {top }}$ is depicted in blue; $f_{+}^{c^{\prime}}$ in beige; $f_{-}^{t o p}$ in green and the $f_{-}^{c^{\prime}}$ in red. The $f_{ \pm}^{\eta}\left(l ; \mu_{-}\right)$curves for $d=9$ $\mu_{-}$model are plotted on Fig.(b): the $f_{+}^{t o p}$ is in blue; $f_{+}^{a}$ is in pink; $f_{+}^{c^{\prime}}$ in beige; $f_{-}^{t o p}$ in green; $f_{-}^{a}$ in yellow and $f_{-}^{c^{\prime}}$ in red.

considered $\lambda<0$ GB DW's as a simple analytic continuation of the GB DW's for positive $\lambda>0$, given by eq. (7.11).

B.1. The $\sigma_{j}^{2}$ real and imaginary parts. We start by rewriting

$$
\sigma_{1}^{2}=\left(\sigma_{3}^{2}\right)^{*}=\left(\sigma_{1}^{2}\right)^{R}+i\left(\sigma_{1}^{2}\right)^{I}
$$

defined by eq. (7.10) in sect.7.3.1, as follows:

$$
\sigma_{1}^{2}=x_{0}+e^{-i \frac{\pi}{4}} \sqrt{\frac{D}{x}} \sqrt{1-i x}
$$

Since $x<1$, we can expand it in a convergent power series:

$$
\begin{aligned}
\sqrt{1-i x} & \equiv \mathcal{P}(x)+i \mathcal{I}(x)=\frac{1}{\Gamma(-1 / 2)} \sum_{n=0}^{\infty} \frac{\Gamma(n-1 / 2)}{n !} i^{n} x^{n} \\
& =\frac{1}{\Gamma(-1 / 2)} \sum_{n=0}^{\infty} \frac{\Gamma(2 n-1 / 2)}{(2 n) !}(-1)^{n} x^{2 n}+i \frac{1}{\Gamma(-1 / 2)} \sum_{n=0}^{\infty} \frac{\Gamma(2 n+1 / 2)}{(2 n+1) !}(-1)^{n} x^{2 n+1} .
\end{aligned}
$$

The series representing the corresponding real $\mathcal{P}(x)$ and imaginary $\mathcal{I}(x)$ parts can be easily resumed to give the following well known functions:

$$
\mathcal{P}(x)=\left(1+x^{2}\right)^{1 / 4} \cos \left(\frac{\arctan (x)}{2}\right), \quad \mathcal{I}(x)=-\frac{x}{\sqrt{2} \sqrt{1+\sqrt{1+x^{2}}}} .
$$

With the above results at hand, we finally get the explicit form of the real and imaginary 
parts for all the $\sigma_{j}^{2}$ :

$$
\begin{aligned}
& \left(\sigma_{1}^{2}\right)^{R}(x)=x_{0}+\sqrt{\frac{D}{2 x}}(\mathcal{P}(x)+\mathcal{I}(x))=\left(\sigma_{3}^{2}\right)^{R}(x), \quad\left(\sigma_{1}^{2}\right)^{I}(x)=\sqrt{\frac{D}{2 \xi}}(\mathcal{I}(x)-\mathcal{P}(x))=-\left(\sigma_{3}^{2}\right)^{I} \\
& \left(\sigma_{2}^{2}\right)^{R}(x)=x_{0}-\sqrt{\frac{D}{2 x}}(\mathcal{P}(x)+\mathcal{I}(x))=\left(\sigma_{4}^{2}\right)^{R}(x) \\
& \left(\sigma_{2}^{2}\right)^{I}(x)=-\sqrt{\frac{D}{2 \xi}}(\mathcal{I}(x)-\mathcal{P}(x))=-\left(\sigma_{4}^{2}\right)^{I}(x) .
\end{aligned}
$$

B.2.The $s_{\text {top }}^{j}$ real and imaginary parts. Taking into account the explicit form of the corresponding GB "topological critical exponents" $s_{\text {top }}^{j}$ (see ref. [5]):

$$
s_{\text {top }}^{j}=\frac{64 B^{2} L_{\text {top }}^{2}}{(d-2)}\left(\sigma_{j}^{2}\right)\left(\sigma_{j}^{2}-x_{0}\right)^{2}=\left(s_{\text {top }}^{j+2}\right)^{*}=\left(s_{\text {top }}^{j}\right)^{R}+i\left(s_{\text {top }}^{j}\right)^{I}, j=1,2
$$

and eqs. (B.1) for the "topological vacua positions" $\sigma_{j}^{2}$, we can separate their real and imaginary parts as follows:

$$
\begin{aligned}
& \left(s_{\text {top }}^{j}\right)^{R} \equiv \frac{64 B^{2}\left(L_{\text {top }}^{2}\right)}{(d-2)}\left\{\left(\sigma_{j}^{2}\right)^{R}\left[\left(\left(\sigma_{j}^{2}\right)^{R}-x_{0}\right)^{2}-\left(\sigma_{j}^{4}\right)^{I}\right]-2\left(\sigma_{j}^{4}\right)^{I}\left(\left(\sigma_{j}^{2}\right)^{R}-x_{0}\right)\right\}, \\
& \left(s_{\text {top }}^{j}\right)^{I} \equiv \frac{64 B^{2}\left(L_{\text {top }}^{2}\right)}{(d-2)}\left\{\left(\sigma_{j}^{2}\right)^{I}\left[\left(\left(\sigma_{j}^{2}\right)^{R}-x_{0}\right)^{2}-\left(\sigma_{j}^{4}\right)^{I}\right]+2\left(\sigma_{j}^{2}\right)^{R}\left(\sigma_{j}^{2}\right)^{I}\left(\left(\sigma_{j}^{2}\right)^{R}-x_{0}\right)\right\}(\mathrm{B} .3
\end{aligned}
$$

B.3.The scale factor real form. In order to derive the real form of the GB scaling factor we are looking for, we first rewrite the following product of two complex conjugate factors

$$
U_{j}(\sigma)=\left(\sigma^{2}-\sigma_{j}^{2}\right)^{-\frac{1}{s_{j}^{t o p}}}\left(\sigma^{2}-\sigma_{j+2}^{2}\right)^{-\frac{1}{s_{j+2}}}, j=1,2,
$$

in terms of certain real functions. By simple complex numbers manipulations we realize that:

$$
\begin{aligned}
U_{j}(\sigma) & =\left(\sigma^{2}-\left(\sigma_{j}^{2}\right)^{R}+i\left(\sigma_{j}^{2}\right)^{I}\right)^{\frac{-s_{j}^{R}+i s_{j}^{I}}{\left(s_{j}^{R}\right)^{2}+\left(s_{j}^{I}\right)^{2}}}\left(\sigma^{2}-\left(\sigma_{j}^{2}\right)^{R}-i\left(\sigma_{j}^{2}\right)^{I}\right)^{\frac{-s_{j}^{R}-i s_{j}^{I}}{\left(s_{j}^{R}\right)^{2}+\left(s_{j}^{I}\right)^{2}}}, \\
& =\left[\left(\sigma^{2}-\left(\sigma_{j}^{2}\right)^{R}\right)^{2}+\left(\sigma_{j}^{4}\right)^{I}\right]^{\frac{-s_{j}^{R}}{\left(s_{j}^{R}\right)^{2}+\left(s_{j}^{I}\right)^{2}}}\left(\frac{1-i \frac{\left(\sigma_{j}^{2}\right)^{I}}{\sigma^{2}-\left(\sigma_{j}^{2}\right)^{R}}}{1+i \frac{\left(\sigma_{j}^{2}\right)^{I}}{\sigma^{2}-\left(\sigma_{j}^{2}\right)^{R}}}\right)^{i \frac{s_{j}^{I}}{\left(s_{j}^{R}\right)^{2}+\left(s_{j}^{I}\right)^{2}}} \\
& =\left[\left(\sigma^{2}-\left(\sigma_{j}^{2}\right)^{R}\right)^{2}+\left(\sigma_{j}^{4}\right)^{I}\right]^{\frac{-s_{j}^{R}}{\left(s_{j}^{R}\right)^{2}+\left(s_{j}^{I}\right)^{2}}} e^{2 \frac{s_{j}^{I}}{\left(s_{j}^{R}\right)^{2}+\left(s_{j}^{I}\right)^{2}} \arctan \left(\frac{\left(\sigma_{j}^{2}\right)^{I}}{\sigma^{2}-\left(\sigma_{j}^{2}\right)^{R}}\right)}
\end{aligned}
$$

Finally, it remains to substitute the above product formula (B.4) into the original generic form (7.7) of the scale factor:

$$
\begin{aligned}
e^{A(\sigma)} & =e^{A_{\infty}}|\sigma|^{-\frac{1}{s_{I R}}}\left|\sigma^{2}-x_{0}\right|^{-\frac{1}{s_{U V}}} G_{\text {top }}^{-} \\
G_{\text {top }}^{-} & =\prod_{j=1}^{2}\left[\left(\sigma^{2}-\left(\sigma_{j}^{2}\right)^{R}\right)^{2}+\left(\left(\sigma_{j}^{2}\right)^{I}\right)^{2}\right]^{-\frac{s_{j}^{R}}{\left(s_{j}^{R}\right)^{2}+\left(s_{j}^{I}\right)^{2}}} \exp \left(\frac{2 s_{j}^{I}}{\left(s_{j}^{R}\right)^{2}+\left(s_{j}^{I}\right)^{2}} \arctan \left(\frac{\left(\sigma_{j}^{2}\right)^{I}}{\sigma^{2}-\left(\sigma_{j}^{2}\right)^{R}}\right)\right),
\end{aligned}
$$


which reproduces the form of the non-singular function $G_{\text {top }}^{-}(\sigma)$ (for $\lambda$ negative), given by eq. (7.11), we have announced in Sect.7.3.1 above. 


\section{References}

[1] J.M.Maldacena, The large N limit of superconformal field theories and supergravity, Adv. Theor. Math. Phys. 2, 231 (1998) [arXiv:hep-th/9711200]; E.Witten, Anti-de Sitter space and holography, Adv. Theor. Math. Phys. 2, 253 (1998) [arXiv:hep-th/9802150]; S.S.Gubser, I.R.Klebanov and A.M.Polyakov, Gauge theory correlators from non-critical string theory, Phys. Lett. B 428, 105 (1998) [arXiv:hep-th/9802109].

[2] J. de Boer,The Holographic Renormalization Group, Fortsch. Phys. 49:339-358(2001), hep-th/0101026; E. Verlinde, H. Verlinde and J. de Boer,On the holographic renormalization group, JHEP 0008:003(2000), hep-th/9912012.

[3] D.Z. Freedman, S.S. Gubser, K. Pilch and N.P. Warner,Renormalization group flows from holography supersymmetry and a c theorem. Adv.Theor.Math.Phys.3(1999),363-417, e-Print: hep-th/9904017; L. Girardello, M. Petrini, M. Porrati and A. Zaffaroni, Novel Local CFT and Exact Results on Perturbations of $\mathrm{N}=4$ Super YangMills from AdS Dynamics, JHEP 12 (1998) 022, hep-th/9810126; M.Bianchi, D.Z.Freedman, K.Skenderis, How to go with an RG flow, JHEP 0108 (2001) 041, e-Print: hep-th/0105276; J. Distler and F. Zamora, Nonsupersymmetric Conformal Field Theories from Stable Anti-de Sitter Spaces, hep-th/9810206; M.Bianchi, D.Z.Freedman, K.Skenderis, Holographic renormalization, Nucl.Phys.B631:159-194,2002, e-Print: hep-th/0112119.

[4] D.Anselmi, L.Girardello, M. Porrati and A. Zaffaroni, A Note on the holographic beta and C functions, Phys.Lett. B481 (2000) 346, hep-th/0002066 [hep-th].

[5] U.Camara dS, C.P. Constantinidis, A.L.Alves Lima and G.M. Sotkov, Domain Walls in Extended Lovelock Gravity, JHEP 1204 (2012) 109, arXiv:1202.4682 [hep-th].

[6] U.Camara dS and G.M.Sotkov, New Massive Gravity Domain Walls, Phys.Lett. B694 (2010) 94-99, e-Print: arXiv:1008.2553 [hep-th].

[7] R. C. Myers, M. F. Paulos and A. Sinha, Holographic studies of quasi-topological gravity, JHEP 1008, 035 (2010) arXiv:1004.2055 [hep-th].

[8] A. Buchel, J. Escobedo, R. C. Myers, M. F. Paulos, A. Sinha and M. Smolkin, Holographic GB gravity in arbitrary dimensions, JHEP 1003 (2010) 111,[arXiv:0911.4257];

[9] R. C. Myers and A. Sinha, Holographic c-theorems in arbitrary dimensions, JHEP 1101, 125 (2011) [arXiv:1011.5819 ]; R. C. Myers and A. Sinha, Seeing a c-theorem with holography,Phys. Rev. D 82, 046006 (2010) arXiv:1006.1263.

[10] X. O. Camanho and J. D. Edelstein, Causality in AdS/CFT and Lovelock theory, JHEP 1006, 099 (2010) [arXiv:0912.1944 ]; A. Buchel, J. Escobedo, R. C. Myers, M. F. Paulos, A. Sinha and M. Smolkin, Holographic GB gravity in arbitrary dimensions, JHEP 1003, 111 (2010). [arXiv:0911.4257]; X. O. Camanho, J. D. Edelstein and M. F. Paulos, Lovelock theories, holography and the fate of the viscosity bound, arXiv:1010.1682.

[11] J. de Boer, M. Kulaxizi and A. Parnachev, AdS7/CFT6, Gauss-Bonnet Gravity, and Viscosity Bound, JHEP 1003, 087 (2010) [arXiv:0910.5347 [hep-th]]; X. O. Camanho and J. D. Edelstein, Causality constraints in AdS/CFT from conformal collider physics and Gauss-Bonnet gravity, JHEP 1004, 007 (2010), [arXiv:0911.3160 [hep-th]]; J. de Boer, M. Kulaxizi and A. Parnachev, Holographic Lovelock Gravities and Black Holes, JHEP 1006, 008 (2010) [arXiv:0912.1877].

[12] A.B.Zamolodchikov, Irreversibility of the Flux of the Renormalization Group in a 2D Field 
Theory, Sov. Phys. JETP Lett. 43(1986)1731;A.B.Zamolodchikov, Renormalization Group And Perturbation Theory Near Fixed Points In Two-Dimensional Field Theory, Sov. J. Nucl. Phys. 46, 1090 (1987) [Yad. Fiz. 46, 1819 (1987)].

[13] J.L.Cardy, Is There a c Theorem in Four-Dimensions?, Phys.Lett. B215 (1988) 749.

[14] D. M. Hofman and J. Maldacena, Conformal collider physics: Energy and charge correlations, JHEP 0805, 012 (2008) [arXiv:0803.1467 [hep-th]].

[15] D.M.Hofman, Higher Derivative Gravity, Causality and Positivity of Energy in a UV complete QFT, Nucl. Phys. B 823, 174 (2009), arXiv:0907.1625 [hep-th]].

[16] A.Buchel and R.C.Myers, Causality of Holographic Hydrodynamics, JHEP 0908, 016 (2009), arXiv:0906.2922 [hep-th].

[17] R. C. Myers and B. Robinson, Black Holes in Quasi-topological Gravity," JHEP 1008, 067 (2010) [arXiv:1003.5357 [gr-qc]].

[18] J. Oliva and S. Ray, A new cubic theory of gravity in five dimensions: Black hole, Birkhoffs theorem and C-function, Class. Quant. Grav. 27, 225002 (2010) ,[arXiv:1003.4773 [gr-qc]];J.Oliva and S.Ray, Classification of Six Derivative Lagrangians of Gravity and Static Spherically Symmetric Solutions,Phys.Rev.D82:124030,2010, arXiv:1004.0737.

[19] D. Lovelock, The Einstein Tensor and Its Generalizations J.Math.Phys. 12, 498.

[20] M. Cvetic and H.H. Soleng, Phys. Rep. 282(1997) 159 and references therein.

[21] D.Z.Friedman,C.Nunez,M.Schnabl,K.Skenderis,Phys.Rev.D69,104 027(2004); M.Cvetic,S.Griffies,S.J.Rey, Nucl. Phys B3811992301.

[22] K.Skenderis and P.K.Townsend,Gravitational stability and Renormalization group flow, PLB $\{468\}\{1999\}\{46\}$,e-Print: hep-th/9909070.

[23] E.T.Akhmedov, A Remark on the AdS / CFT correspondence and the renormalization group flow, Phys.Lett. B442 (1998) 152-158 hep-th/9806217 [hep-th]; E.T.Akhmedov, Notes on multitrace operators and holographic renormalization group, hep-th/0202055 [hep-th].

[24] P. Kovtun, D. T. Son and A. O. Starinets, Viscosity in strongly interacting quantum field theories from black hole physics, Phys. Rev. Lett. 94, 111601 (2005), [arXiv:hep-th/0405231]; P. Kovtun, D. T. Son and A. O. Starinets, Holography and hydrodynamics: Diffusion on stretched horizons, JHEP 0310, 064 (2003), [arXiv:hep-th/0309213]; A. Buchel and J. T. Liu, Universality of the shear viscosity in supergravity, Phys. Rev.Lett. 93, 090602 (2004), [arXiv:hep-th/0311175];A. Buchel, On universality of stress-energy tensor correlation functions in supergravity, Phys. Lett. B 609, 392 (2005), [arXiv:hep-th/0408095]; A. Buchel, J. T. Liu and A. O. Starinets, Coupling constant dependence of the shear viscosity in $N=4$ supersymmetric Yang-Mills theory, Nucl. Phys. B 707, 56 (2005),[arXiv:hep-th/0406264].

[25] Y. Kats and P. Petrov, Effect of curvature squared corrections in AdS on the viscosity of the dual gauge theory, JHEP 0901, 044 (2009) [arXiv:0712.0743 [hep-th]];R. C. Myers, M. F. Paulos and A. Sinha, Holographic Hydrodynamics with a Chemical Potential, JHEP 0906, 006 (2009) [arXiv:0903.2834 [hep-th]];X.-H. Ge and S.-J. Sin, Shear viscosity, instability and the upper bound of the Gauss-Bonnet coupling constant, JHEP 05 (2009) 051

[arXiv:0903.2527];X.-H. Ge, S. J. Sin, et al, Shear viscosity and instability from third order Lovelock gravity, Phys. Rev. D, 80,104019 (2009).

[26] M. Brigante, H. Liu, R. C. Myers, S. Shenker and S. Yaida, Viscosity Bound Violation in Higher Derivative Gravity, Phys. Rev. D 77, 126006 (2008) [arXiv:0712.0805]; M. Brigante, 
H. Liu, R. C. Myers, S. Shenker and S. Yaida, The Viscosity Bound and Causality Violation, Phys. Rev. Lett. 100, 191601 (2008) [arXiv:0802.3318].

[27] D. G. Boulware and S. Deser, String Generated Gravity Models, Phys. Rev. Lett. 55 (1985) 2656.

[28] J. T. Liu, W. Sabra and Z. Zhao, Holographic c-theorems and higher derivative gravity, arXiv:1012.3382 [hep-th];A. Sinha, On higher derivative gravity, c-theorems and cosmology,Class. Quant.Grav. 28, 085002 (2011) [arXiv:1008.4315 [hep-th]]; M. F. Paulos, Holographic phase space: c-functions and black holes as renormalization group flows, JHEP 1105, 043 (2011) [arXiv:1101.5993 [hep-th]];Mohamed M. Anber and David Kastor, C-Functions in Lovelock Gravity, JHEP 0805:061,2008, arXiv:0802.1290 [hep-th].

[29] P.Breitenlohner and D.Z.Freedman,PLB $\{115\}\{1982\}\{197\}$; Ann.Phys.144(1982)249.

[30] I.R.Klebanov and E. Witten, AdS/CFT correspondence and symmetry breaking, Nucl. Phys., B556:89114, 1999.

[31] A.M.Polyakov, Gauge Fields and Strings, Chur, Switzerland: Harwood (1987), Contemporary Concepts in Physics 3.

[32] A.M.Polyakov, Conformal symmetry of critical fluctuations, JETP Lett. 12 (1970) 381.

[33] J.L.Cardy, Scaling and Renormalization in Statistical Physics,Cambridge Lecture notes in Physics 5, Cambridge University Press 1996; J.L.Cardy, Conformal Invariance And Statistical Mechanics, 1988 Les Houches lectures.

[34] U.Camara dS, C.P.Constantinidis and G.M.Sotkov, New Massive Gravity Holography; arXiv:1009.2665.

[35] G.Mussardo, Statistical Field Theory, Oxford University Press Inc., New York, 2010.

[36] A.Sinha, On the new massive gravity and AdS/CFT, JHEP 1006, 061 (2010) [arXiv:1003.0683].

[37] H.L.C. Louzada, U. Camara dS and G.M. Sotkov, Massive 3D Gravity Big-Bounce, Phys. Lett. B 686 (2010) 268,[arXiv:1001.3622 [hep-th]].

[38] U. Camara dS and G.Sotkov; Geometry of the new massive gravity domain walls; PoS ICF2010(2010)026.

[39] V.K.Dobrev, G.Mack, V.B.Petkova, S.G.Petrova and I.T.Todorov, Harmonic Analysis On The N-Dimensional Lorentz Group And Its Application To Conformal Quantum Field Theory, Lecture Notes in Physics 63 (Berlin 1977, 280 pg); I.T.Todorov, M.C.Mintchev and V.B.Petkova, Conformal Invariance In Quantum Field Theory, Pisa, Italy: Sc. Norm. Sup. 1978, 273p.

[40] M.J.Duff, Observations On Conformal Anomalies, Nucl. Phys. B 125, 334 (1977); M.J.Duff, Twenty years of the Weyl anomaly, Class. Quant. Grav. 11, 1387 (1994), [arXiv:hep-th/9308075].

[41] N.D.Birrel and P.C.W.Davies, Quantum fields in curved space, Cambridge University Press, Cambridge, 1982.

[42] D.Anselmi, D.Z.Freedman, M.T.Grisaru and A.A.Johansen, Nonperturbative formulas for central functions of supersymmetric gauge theories, Nucl. Phys. B526 (1998) 543, hep-th/9708042;D.Anselmi, J.Erlich, D.Z.Freedman and A.A. Johansen, Positivity 
constraints on anomalies in supersymmetric gauge theories, Phys. Rev. D 57, 7570 (1998), arXiv:hep-th/9711035.

[43] D.Anselmi, Anomalies, unitarity and quantum irreversibility, Annals Phys. 276, 361 (1999), [hep-th/9903059].

[44] I.R.Klebanov, S.S.Pufu and B.R.Safdi, F-Theorem without Supersymmetry, JHEP 1110 (2011) 038, arXiv:1105.4598 [hep-th].

[45] A.A.Belavin ,A.M.Polyakov and A.B.Zamolodchikov,Infinite Conformal Symmetry in Two-Dimensional Quantum Field Theory, NPB $\{241\}\{1984\}\{333\}$.

[46] D.Anselmi,Inequalities for trace anomalies, length of the RG flow, distance between the fixed points and irreversibility, Class.Quant.Grav. 21 (2004) 29-50 hep-th/0210124 [hep-th]; D.Anselmi, Central functions and their physical implications, JHEP 9805 (1998) 005, hep-th/9702056 [hep-th]

[47] D.Anselmi, Exact results on quantum field theories interpolating between pairs of conformal field theories, Talk given at Conference: C99-05-27, p.551-559, e-Print: hep-th/9910255 [hep-th];D.Anselmi, Towards the classification of conformal field theories in arbitrary dimension, Phys.Lett. B476 (2000) 182-187, hep-th/9908014 [hep-th]; D.Anselmi, Quantum irreversibility in arbitrary dimension, Nucl.Phys. B567 (2000) 331-359, hep-th/9905005 [hep-th].

[48] D.Anselmi, Quantum conformal algebras and closed conformal field theory, Nucl.Phys. B554 (1999) 415-436, hep-th/9811149 [hep-th]

[49] S.J. Hathrell, Trace anomalies and $\lambda \phi^{4}$ theory in curved space, Ann. Phys. (N.Y.) 139 (1982) 136.

[50] A.Cappelli, J.I.Latorre and X.Vilasis-Cardona, Renormalization Group Patterns and C-Theorem in More Than Two Dimensions, Nucl. Phys. B 376, 510 (1992), arXiv:hep-th/9109041.

[51] H.Osborn, Derivation of a Four-Dimensional c-theorem, Phys. Lett. B 222, 97 (1989).

[52] G.M.Sotkov and R.P.Zaikov, Conformal Invariant Two Point and Three Point Functions for Fields with Arbitrary Spin, Rept.Math.Phys. 12 (1977) 375; G.M.Sotkov and R.P.Zaikov, On The Structure Of The Conformal Covariant N Point Functions, Rept.Math.Phys. 19 (1984) 335 .

[53] A. Buchel, R.C. Myers and A. Sinha, Beyond eta/s = 1/4 pi, JHEP 0903 (2009) 084, arXiv:0812.2521 [hep-th].

[54] Z.Komargodski and A.Schwimmer, On Renormalization Group Flows in Four Dimensions, arXiv:1107.3987[hep-th]; Z.Komargodski, The Constraints of Conformal Symmetry on RG Flows, arXiv:1112.4538 [hep-th].

[55] M.A.Luty, J.Polchinski and R.Rattazzi, The a-theorem and the Asymptotics of 4D Quantum Field Theory, arXiv:1204.5221 [hep-th].

[56] S.Deser and A.Schwimmer, Geometric classification of conformal anomalies in arbitrary dimensions, Phys. Lett. B 309, 279 (1993), arXiv:hep-th/9302047.

[57] D.Z.Freedman, M.Headrick and A.Lawrence, On closed string tachyon dynamics, Phys.Rev. D73 (2006) 066015, arXiv:hep-th/0510126. 
[58] H.Osborn and A.C.Petkou, Implications of Conformal Invariance in Field Theories for General Dimensions, Annals Phys. 231, 311 (1994) [arXiv:hep-th/9307010]; J.Erdmenger and H.Osborn, Conserved currents and the energy-momentum tensor in conformally invariant theories for general dimensions, Nucl. Phys. B 483, 431 (1997)

[arXiv:hep-th/9605009];J.I.Latorre, H.Osborn, Positivity and the energy momentum tensor in quantum field theory, Nucl.Phys. B511 (1998) 737, hep-th/9703196 [hep-th]

[59] I.Jack and H.Osborn, Background Field Calculations In Curved Space-time. 1. General Formalism And Application To Scalar Fields, Nucl.Phys. B234 (1984) 331; I.Jack and H. Osborn, Analogues for the $\mathrm{C}$ theorem for four-dimensional renormalizable field theories, Nucl. Phys. B 343 (1990) 647.

[60] O. Miskovic and R. Olea, Counterterms in Dimensionally Continued AdS Gravity, JHEP 0710 (2007) 028 [arXiv:0706.4460 ]; G. Kofinas and R. Olea, Universal regularization prescription for Lovelock AdS gravity, JHEP 0711, 069 (2007) [arXiv:0708.0782]; G. Kofinas and R. Olea, Universal Kounterterms in Lovelock AdS gravity, Fortsch. Phys. 56, 957 (2008) [arXiv:0806.1197 ].

[61] E. Dyer, K. Hinterbichler, Boundary Terms, Variational Principles and Higher Derivative Modified Gravity, Phys.Rev. D79 (2009) 024028 ,e-Print: arXiv:0809.4033 [gr-qc].

[62] Ling-Yan Hung, Robert C. Myers, Michael Smolkin,Some Calculable Contributions to Holographic Entanglement Entropy, JHEP 1108:039,2011, arXiv:1105.6055 [hep-th]; L. Y. Hung, R. C. Myers and M. Smolkin, On Holographic Entanglement Entropy and Higher Curvature Gravity, arXiv:1101.5813 [hep-th]; J. de Boer, M. Kulaxizi and A. Parnachev, Holographic Entanglement Entropy in Lovelock Gravities, arXiv:1101.5781 [hep-th];T.Albash, C.V.Johnson, Holographic Entanglement Entropy and Renormalization Group Flow, arXiv:1110.1074 [hep-th]; Robert C. Myers and Ajay Singh; Comments on Holography Entanglement Entropic and RG Flows; arXiv:1202.2068.

[63] A.Buchel, Bulk viscosity of gauge theory plasma at strong coupling, Phys.Lett. B663 (2008) 286-289, arXiv:0708.3459 [hep-th]; A.Buchel, Violation of the holographic bulk viscosity bound, Phys.Rev. D85 (2012) 066004, arXiv:1110.0063 [hep-th]; A.Buchel, Umut Gursoy and Elias Kiritsis, Holographic bulk viscosity: GPR versus EO, JHEP 1109 (2011) 095, arXiv:1104.2058 [hep-th].

[64] M.H. Dehghani, A. Bazrafshan, R.B. Mann, M.R. Mehdizadeh, M. Ghanaatian, Black Holes in Quartic Quasitopological Gravity, e-Print: arXiv:1109.4708 [hep-th].

[65] S.Ryu and T.Takayanagi, Holographic derivation of entanglement entropy from AdS/CFT, Phys. Rev. Lett. 96, 181602 (2006) [arXiv:hep-th/0603001]; S.Ryu and T.Takayanagi, Aspects of holographic entanglement entropy, JHEP 0608, 045 (2006) [arXiv:hep-th/0605073].

[66] M.Kulaxizi and Andrei Parnachev, Energy Flux Positivity and Unitarity in CFTs, Phys.Rev.Lett. 106 (2011) 011601, arXiv:1007.0553 [hep-th].

[67] V.A.Miransky and K.Yamawaki, Conformal phase transition in gauge theories, Phys. Rev. D 55 (1997) 5051, Erratum-ibid. D 56 (1997) 3768, arXiv:hep-th/9611142.

[68] D.B.Kaplan, J.-W.Lee, D.T.Son and M.A.Stephanov, Conformality Lost, Phys. Rev. D80, 125005 (2009), arXiv:0905.4752[hep-th].

[69] D.Kutasov, J.Lin and A.Parnachev, Conformal Phase Transitions at Weak and Strong Coupling, Nucl.Phys. B858 (2012) 155-195, arXiv:1107.2324 [hep-th]. 
[70] N.Seiberg, Exact results on the space of vacua of four-dimensional SUSY gauge theories, Phys. Rev. D 49 (1994) 6857, arXiv:hep-th/9402044; N.Seiberg, Electric - magnetic duality in supersymmetric nonAbelian gauge theories, Nucl. Phys. B435 (1995) 129, arXiv:hep-th/9411149.

[71] Matti Jarvinen and Elias Kiritsis, Holographic Models for QCD in the Veneziano Limit, JHEP 1203 (2012) 002, arXiv:1112.1261 [hep-ph]; E.Kiritsis, Dissecting the string theory dual of QCD, Fortsch. Phys. 57 (2009) 396 arXiv:0901.1772[hep-th]; U. Gursoy, E.Kiritsis, L.Mazzanti and F. Nitti, Holography and Thermodynamics of 5D Dilaton-gravity, JHEP 0905 (2009) 033, arXiv:0812.0792[hep-th].

[72] G. Veneziano, U(1) Without Instantons, Nucl. Phys. B 159 (1979) 213; T. Banks and A. Zaks, On the Phase Structure of Vector-Like Gauge Theories with Massless Fermions, Nucl. Phys. B196 (1982) 189.

[73] Robert C. Myers and Ajay Singh; Comments on Holography Entanglement Entropic and RG Flows; arXiv:1202.2068.

[74] Sang-Heon Yi, Born-Infeld Type Extension of (Non-)Critical Gravity, arXiv:1203.3383 [hep-th].

[75] Miguel F. Paulos and Andrew J. Tolley, Massive Gravity Theories and limits of Ghost-free Bigravity models, arXiv:1203.4268 [hep-th].

[76] U.Camara dS and G.M.Sotkov, Domain Walls and a/c-Theorems in Quasi-Topological Gravity coupled to Sigma models (in preparation). 